University of Louisville

ThinkIR: The University of Louisville's Institutional Repository

Electronic Theses and Dissertations

$12-2018$

\title{
Influence of bilberry-derived anthocyanidins on key regulators of colorectal cancer development.
}

Ashley M. Mudd

University of Louisville

Follow this and additional works at: https://ir.library.louisville.edu/etd

Part of the Alternative and Complementary Medicine Commons, and the Pharmacy and Pharmaceutical Sciences Commons

\section{Recommended Citation}

Mudd, Ashley M., "Influence of bilberry-derived anthocyanidins on key regulators of colorectal cancer development." (2018). Electronic Theses and Dissertations. Paper 3129.

https://doi.org/10.18297/etd/3129

This Doctoral Dissertation is brought to you for free and open access by ThinkIR: The University of Louisville's Institutional Repository. It has been accepted for inclusion in Electronic Theses and Dissertations by an authorized administrator of ThinkIR: The University of Louisville's Institutional Repository. This title appears here courtesy of the author, who has retained all other copyrights. For more information, please contact thinkir@louisville.edu. 


\title{
INFLUENCE OF BILBERRY-DERIVED ANTHOCYANIDINS ON KEY REGULATORS OF COLORECTAL CANCER DEVELOPMENT
}

\author{
By \\ Ashley M. Mudd \\ A Dissertation submitted to the faculty of the School of Medicine of the University \\ of Louisville in partial fulfillment of the requirements for the degree of \\ Doctor of Philosophy in Pharmacology and Toxicology \\ Department of Pharmacology and Toxicology \\ University of Louisville \\ Louisville, Kentucky
}

December 2018 



\title{
INFLUENCE OF BILBERRY-DERIVED ANTHOCYANIDINS ON KEY REGULATORS OF COLORECTAL CANCER DEVELOPMENT
}

\author{
By \\ Ashley M. Mudd \\ A Dissertation approved on \\ August 24th, 2018 \\ By the following Dissertation committee:
}

Ramesh C. Gupta, Ph.D.

Nejat K. Egilmez, Ph.D.

David W. Hein, Ph.D.

Radha Munagala, Ph.D.

David J. Schultz, Ph.D.

Vivek R. Sharma, M.D. 


\section{DEDICATION}

This dissertation is dedicated to my family for all of their support and encouragement and my two 'little scientists'. 


\section{ACKNOWLEDGEMENTS}

I would like to acknowledge and thank my mentor Dr. Ramesh C. Gupta for all of his support, patience and encouragement in all of my scientific endeavors. I would also like to thank Dr. Nejat K. Egilmez and Dr. Tao Gu for all of their help with the animal studies contained within this dissertation, their advice, support and encouragement. I would also like to thank my committee members including Dr. Nejat K. Egilmez, Dr. David W. Hein, Dr. Radha Munagala, Dr. David J. Schultz and Dr. Vivek R. Sharma for all of their support. I would also like to thank all of the members of the Gupta lab, 3P Biotechnologies and Egilmez lab for their training, patience and willingness to always help. I would also like to thank Cady E. Barbour for her help performing the in vitro western blot experiments probing PD-L1 and Jeyaprakash Jeyabalan for determining the purity of the bilberryderived Anthos. 


\begin{abstract}
INFLUENCE OF BILBERRY-DERIVED ANTHOCYANIDINS ON KEY REGULATORS OF

COLORECTAL CANCER DEVELOPMENT
\end{abstract}

Ashley Marie Mudd

August $24^{\text {th }} 2018$

Colorectal cancer $(\mathrm{CRC})$ is the third leading cause of cancer-related deaths within the United States. A variety of factors including exposure to environmental carcinogens, such as benzo[a]pyrene $(\mathrm{B}[\mathrm{a}] \mathrm{P})$, dysbiosis of the gut microbiome and hereditary influences, including APC mutations, have been shown to lead to an increased risk of developing colorectal cancer. Given the recent alarming rise in cases of colorectal cancer diagnosed in individuals under the age of 40 , further insight into developing novel and effective prevention and treatment strategies are warranted. The family of plant pigments known as the anthocyanins has been identified with a variety of health benefits including chemopreventive and therapeutic effects. However, a limitation to current clinical applications of anthocyanins is the high doses that are required. In order to overcome this barrier we tested the active moiety, anthocyanidins, native bilberry mixture of anthocyanidins (Anthos), at various doses for chemopreventive and therapeutic effects against colorectal cancer both in vitro and in vivo. Anthos treatment led to favorable modulation of several key contributors to the development of CRC including Src/EGFR pathway, Phase I/II enzyme expression and the inflammatory microenvironment, with special emphasis on the expression of PD-L1, a key immune checkpoint protein both in vitro and in vivo using an $\mathrm{Apc}^{\mathrm{Min} /+} \mathrm{ETBF}$ mouse tumor model. These results provide a promising outlook on the impact of berry Anthos for treatment and prevention of bacteria and $\mathrm{B}[\mathrm{a}] \mathrm{P}$-driven colorectal cancer. Results from this study also 
provide novel mechanistic insight into the chemopreventive and therapeutic activities of Anthos. 


\section{TABLE OF CONTENTS}

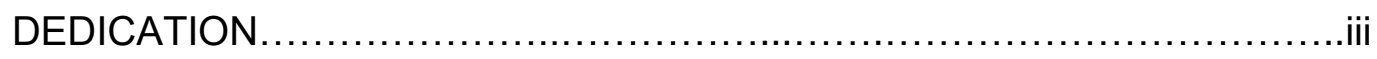

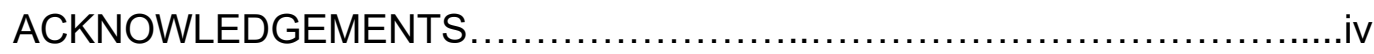

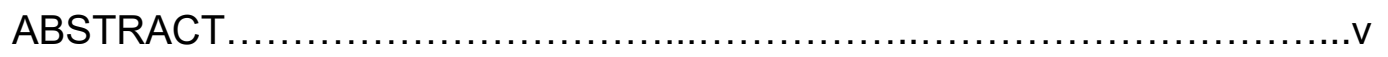

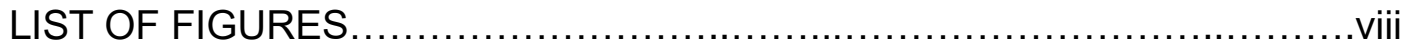

Chapter 1: PREFACE, BACKGROUND AND INTRODUCTION

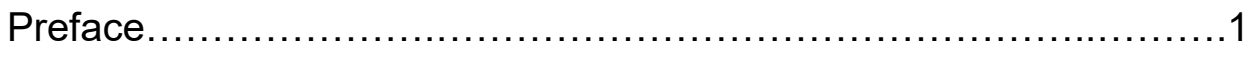

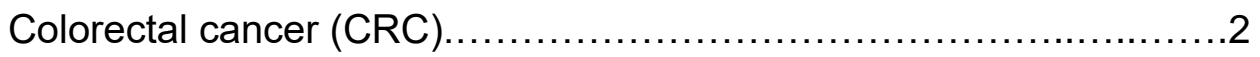

Familial Adenomatous Polyposis (FAP) ...............................15

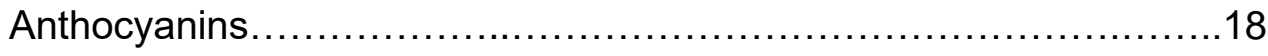

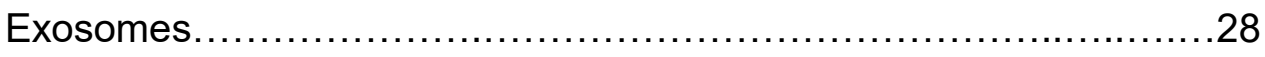

Chapter 2: Novel insight into the prevention and treatment of colorectal cancer and familial adenomatous polyposis by anthocyanidins

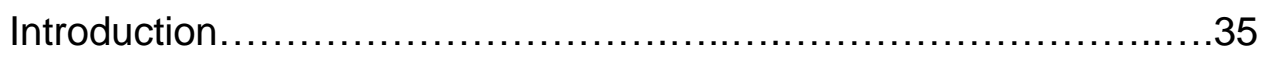

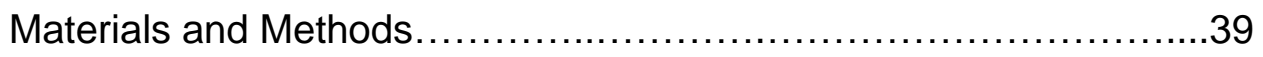

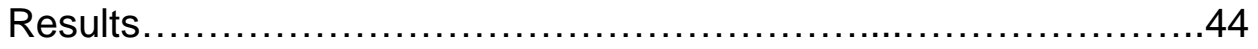

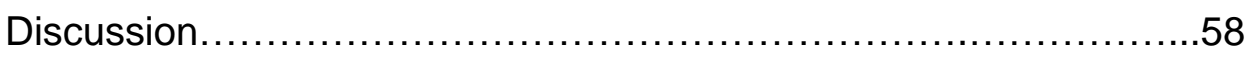

Chapter 3: The role of anthocyanidins in reequilibrating metabolic shifts induced by dysbiosis of the gut microbiome and the ubiquitous environmental carcinogen, Benzo[a]pyrene 


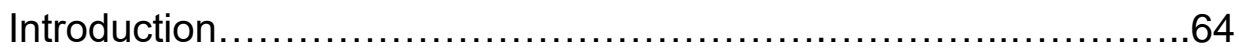

Materials and Methods ................................................

Results ............................................................

Discussion ..............................................................

Chapter 4: Modulation of colon inflammatory environment by bilberry-derived anthocyanidins

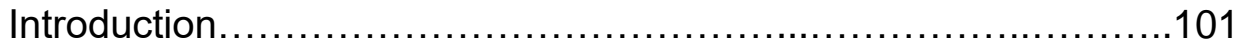

Materials and Methods ..............................................108

Results ............................................................... 111

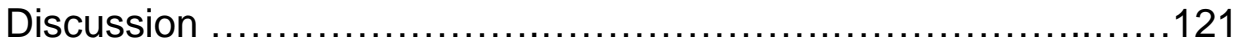

Chapter 5: SUMMARY AND CONCLUSIONS..............................127

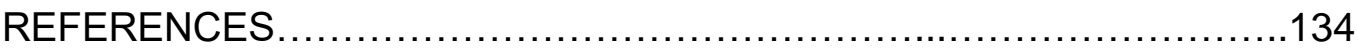

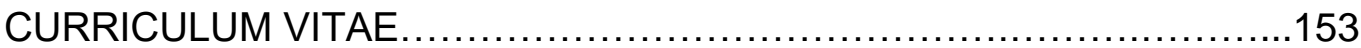




\section{LIST OF FIGURES}

\section{FIGURE:}

1. Distribution of estimated new cases of cancer \& deaths for $2018 \ldots \ldots \ldots \ldots . . .3$

2. Adenoma-carcinoma sequence in CRC.................................13

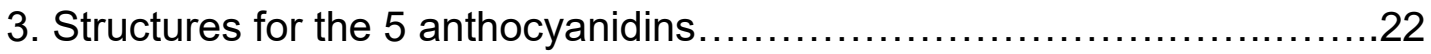

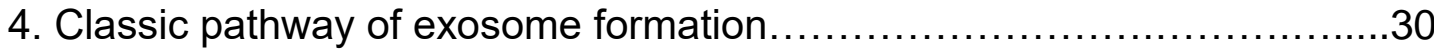

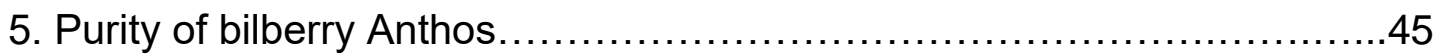

6. Antiproliferative activity of Anthocyanidins versus anthocyanins............46

7. Antiproliferative activity of native bilberry Anthos..........................47

8. Anti-polyp and anti-tumor activity of bilberry derived Anthos..................49

9. Changes in EGFR \& Src phosphorylation after Bilberry Anthos

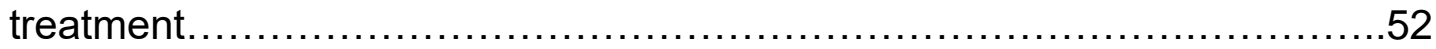

10. In vivo changes in Src and EGFR pathways following treatment with Anthos .54

11. Representative heatmap for in vivo changes in Src and EGFR pathways following treatment with Anthos .55

12. Pathway involving modulation of Src and EGFR phosphorylation status and downstream targets .57

13. Metabolism of benzo[a]pyrene to carcinogenic BPDE vs. detoxification pathway. 66

14. Antiproliferative activity of Exosomes against colon normal cells and cancer cell lines .78 
15. Antiproliferative activity of Anthos and ExoAnthos against colon normal cells and cancer cells in vitro

16. Anti-tumor activities of bilberry-derived Anthos and ExoAnthos against colon tumors in vivo

17. In vivo changes in phase I and II enzyme expression and related xenobiotic sensing nuclear receptors in normal colon tissue following treatment with ETBF bacteria, bilberry-derived Anthos or ExoAnthos in colon tissue

18. Representative heatmaps for In vivo changes in phase I \& II enzyme expression and related xenobiotic sensing nuclear receptors in colon tissue

19. In vivo changes in phase I and II enzyme expression and related xenobiotic sensing nuclear receptors in normal colon tissue following treatment with ETBF bacteria, bilberry-derived Anthos or ExoAnthos in liver tissue .88

20. Representative heatmaps for In vivo changes in phase I \& II enzyme expression and related xenobiotic sensing nuclear receptors in liver tissue .89

21. Impact of in vivo Anthos treatment on phase II enzyme expression in colon tumor tissue .92

22. Changes in expression of key phase I and II enzymes and related nuclear receptors following treatment with $\mathrm{B}[\mathrm{a}] \mathrm{P}$ versus $\mathrm{B}[\mathrm{a}] \mathrm{P}+$ Anthos.

23. In vivo Anthos dose escalation study 112 24. Images of colon sections taken from control and Anthos (high dose) treated animals 
25. In vivo changes in PD-L1 related expression and upstream pathways

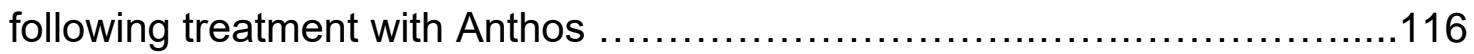

26. Representative heatmap for in vivo changes in PD-L1 related expression and upstream pathways following treatment with Anthos........................117

27. Tentative pathways for modulation of PD-L1 expression ...................119

28. In vitro changes in PD-L1 expression and related EGFR phosphorylation status following Anthos and anthocyanidin treatment ........................120 


\section{LIST OF TABLES}

\section{TABLE}

1. Raw density values calculated for Src/EGFR pathway western blot analysis .56

2. Tabulated selectivity values for colon cancer and normal cells treated with Anthos and ExoAnthos 80

3. Raw density values calculated for western blot analysis of phase I \& II enzyme expression and related xenobiotic sensing nuclear receptors in normal colon tissue .86

4. Raw density values calculated for western blot analysis of phase I \& II enzyme expression and related xenobiotic sensing nuclear receptors in liver tissue. .90

5. Tumor numbers for in vivo Anthos dose escalation study 113

6. Raw density values calculated for PD-L1/inflammation pathway western blot analysis 
CHAPTER 1:

PREFACE, BACKGROUND \& INTRODUCTION

\section{Preface}

Previously thought to have been a disease on the decline, colorectal cancer (CRC) has recently made a comeback in younger individuals. Of these cases of $\mathrm{CRC}$, the majority are in the distal and rectal regions. The exact cause(s) for this alarming uptick have yet to be identified. Given this alarming rise, there is an urgent need for research in both the prevention and treatment of CRC. With this in mind, the focus of my doctoral research has been to utilize berry-derived Anthos and exosomal formulation of Anthos (ExoAnthos) for the prevention and treatment of a bacterially-driven model of CRC. Similar to the majority of individuals that have been diagnosed with $\mathrm{CRC}$ in the younger generations, this model develops tumors in the distal colon. A major component of the work presented here has been to unravel the highly interrelated and complex mechanism of action for how Anthos achieves highly significant reductions in tumor burden as well as to determine how Anthos exerts protection against the ubiquitous environmental carcinogen benzo[a]pyrene $(B[a] P)$. Although, it should be noted that gaining a complete understanding for how Anthos functions would need a lifetime in research rather than the finite span that defines an individual's 
time as a graduate student. Thus, the picture presented here is but a small albeit compelling piece of the overall puzzle.

\section{$\underline{\text { CRC }}$}

Classified as the third most common form of cancer diagnosed in men and women, $\mathrm{CRC}$ is the third leading class for cancer-related deaths within the United States. In fact, according to the American Cancer Society, an estimated 140,250 individuals will be diagnosed and 50,630 will die from CRC in the US in 2018 alone. Therefore, given the prevalence and lethality of $\mathrm{CRC}$, there is an urgent need for investigating not only chemotherapeutic alternatives but also chemopreventive strategies for this disease.

\section{Influences on the development of CRC}

It is estimated that cases of CRC will increase by approximately $60 \%$ or 2.2 million new cases and 1.1 million deaths by 2030 [1]. Several factors have been found to impact an individual's chance of developing CRC including age, race, gender [2], lifestyle and diet [3]. According to the American Cancer Society, the lifetime risk of developing $\mathrm{CRC}$ is about 1 in 22 (4.49\%) for men and 1 in 24 $(4.15 \%)$ for women. The highest incidence rates have been found in countries with high or very high human health indexes (HDI) such as North America, Europe, Australia and New Zealand and the lowest incidence rates have been found in Africa and South-Central Asia [4]. It should also be noted that although the rates of CRC have been increasing recently in other parts of Asia, India has maintained a low incidence of CRC. 
Estimated New Cancer Cases* in the US in 2018

\begin{tabular}{|c|c|c|c|c|c|}
\hline & & $\begin{array}{l}\text { Males } \\
\mathbf{8 5 6 , 3 7 0}\end{array}$ & $\begin{array}{l}\text { Females } \\
878,980\end{array}$ & & \\
\hline Prostate & $19 \%$ & & & $30 \%$ & Breast \\
\hline Lung \& bronchus & $14 \%$ & & & $13 \%$ & Lung \& bronchus \\
\hline Colon \& rectum & $9 \%$ & & & $7 \%$ & Colon \& rectum \\
\hline Urinary bladder & $7 \%$ & & & $7 \%$ & Uterine corpus \\
\hline Melanoma of skin & $6 \%$ & & & $5 \%$ & Thyroid \\
\hline Kidney \& renal pelvis & $5 \%$ & & & $4 \%$ & Melanoma of skin \\
\hline $\begin{array}{l}\text { Non-Hodgkin } \\
\text { lymphoma }\end{array}$ & $5 \%$ & & & $4 \%$ & $\begin{array}{l}\text { Non-Hodgkin } \\
\text { lymphoma }\end{array}$ \\
\hline Oral cavity \& pharynx & $4 \%$ & & & $3 \%$ & Pancreas \\
\hline Leukemia & $4 \%$ & & & $3 \%$ & Leukemia \\
\hline $\begin{array}{l}\text { Liver \& intrahepatic } \\
\text { bile duct }\end{array}$ & $4 \%$ & & & $3 \%$ & Kidney \& renal pelvis \\
\hline All other sites & $22 \%$ & & & $21 \%$ & All other sites \\
\hline
\end{tabular}

\section{Estimated Cancer Deaths in the US in 2018}

\begin{tabular}{|c|c|c|c|c|c|}
\hline & & $\begin{array}{c}\text { Males } \\
323,630\end{array}$ & $\begin{array}{c}\text { Females } \\
286,010\end{array}$ & & \\
\hline Lung \& bronchus & $26 \%$ & & & $25 \%$ & Lung \& bronchus \\
\hline Prostate & $9 \%$ & & & $14 \%$ & Breast \\
\hline Colon \& rectum & $8 \%$ & & & $8 \%$ & Colon \& rectum \\
\hline Pancreas & $7 \%$ & & & $7 \%$ & Pancreas \\
\hline $\begin{array}{l}\text { Liver \& intrahepatic } \\
\text { bile duct }\end{array}$ & $6 \%$ & & & $5 \%$ & Ovary \\
\hline Leukemia & $4 \%$ & & & $4 \%$ & Uterine corpus \\
\hline Esophagus & $4 \%$ & & & $4 \%$ & Leukemia \\
\hline Urinary bladder & $4 \%$ & & & $3 \%$ & $\begin{array}{l}\text { Liver \& intrahepatic } \\
\text { bile duct }\end{array}$ \\
\hline $\begin{array}{l}\text { Non-Hodgkin } \\
\text { lymphoma }\end{array}$ & $4 \%$ & & & $3 \%$ & Non-Hodgkin lymphoma \\
\hline Kidney \& renal pelvis & $3 \%$ & & & $3 \%$ & $\begin{array}{l}\text { Brain \& other nervous } \\
\text { system }\end{array}$ \\
\hline All other sites & $24 \%$ & & & $24 \%$ & All other sites \\
\hline
\end{tabular}

Figure 1: Distribution of estimated new cases of cancer and cancer deaths for 2018 by site, figure from American Cancer Society, Cancer Facts \& Figures 2018. 
The low rate of $C R C$ in this region has been hypothesized to be linked to variety of factors such as the widespread consumption of fruits and vegetables, vegetarianism and the use of spices such as turmeric (curry powder), which is rich in the protective phytochemical, curcumin $[5,6]$. The important role of one's diet on the likelihood of developing CRC was previously identified in a casecontrol study in Greece which found that individuals who consumed a diet that was high meat/low vegetable versus high vegetable/low meat showed a risk ratio of about 8 [7]. Additional, more recent studies assessing the influence of dietary vegetable and particularly fruit intake on $\mathrm{CRC}$ prevention have found similar protective benefits in individuals from Sweden, China, South Korea and the United States [8-11] Overall, these epidemiological findings highlight the protective role that vegetable and particularly fruit consumption may have against the development of CRC and may be associated with the geographic differences in incidence noted above.

Although the incidence of CRC has been decreasing since the mid to late 1980 s for individuals over 55 years old, a recent study has found a rather disconcerting uptick in the CRC incidence for individuals below 55 years old in the US [12]. A particularly concerning trend was noted for younger individuals, with individuals born circa 1990 having a risk of developing colon cancer that is double that of an individual born circa 1950 and for rectal cancer the risk was four-fold higher. Moreover, a greater number of the younger cases were found in the distal colon as opposed to the proximal colon. This is somewhat surprising considering that tumors in the distal colon have been decreasing overall in recent years, while 
tumors of the proximal colon have become more common in the 55 years or older portion of the population [12]. The exact causes for this recent increase in younger individuals have yet to be defined. However, a number of factors have been shown to contribute to the development of CRC. The below summary provides an overview of some of the key environmental as well as genetic factors that are related to the topic of this dissertation and that have been found to impact the development of CRC.

\section{The impact of diet and lifestyle on CRC risk}

The important role that diet and lifestyle play not only on one's overall health but on the chance of developing CRC has been found in a number of studies. Furthermore, it should be noted that chronic inflammation has been shown to be a significant etiological factor in the development of colorectal adenocarcinoma [13]. It has been shown in both males and females that a link exists between consuming a high inflammatory diet, i.e. a diet rich in animal fat, alcohol et cetera and low in fruits and vegetables, and developing CRC [3]. Moreover, results from the same study also supported the benefits of an anti-inflammatory diet, which include plant-based foods which are a rich source of phytochemicals, over a proinflammatory diet, which includes fried foods, highly processed foods etc., as a means of decreasing the risk of developing CRC [3]. Additionally, coffee, which contains a variety of bioactive compounds including polyphenols, melanoidins, diterpenes, is believed to also lower the risk of developing CRC [14]. Epidemiological studies comparing coffee drinkers vs. non-drinkers showed a $26 \%$ lower odds of developing CRC [14]. There also appeared to be a dose- 
dependent trend for both colon and rectal cancers. It was also noted that decaffeinated as well as boiled coffee showed an inverse association (OR, 0.82; 95\% Cl: 0.68-0.99; $\mathrm{P}=0.04)$ and $(\mathrm{OR}, 0.82 ; 95 \% \mathrm{Cl}: 0.71-0.94 ; \mathrm{P}=0.004)$, respectively [14]. In addition to coffee, it has also been shown that regular green tea consumption (at least three times per week for a minimum of 6 months), which is a rich source of antioxidant tea polyphenols, reduces the risk of CRC $(\mathrm{HR}=0.54,95 \% \mathrm{Cl}: 0.34-0.86)$. Furthermore, it was noted that as green tea consumption was increased risk of $C R C$ decreased $\left(P_{\text {trend }}=0.01\right)$ [15].

In addition to dietary influences, another important factor that must be considered is the long term use of medications such as statins, aspirin and non-aspirin nonsteroidal anti-inflammatory drugs which are also believed to lower the risk of developing CRC. For example statins which are believed to inhibit 3-hydroxy3methylglutaryl coenzyme A (HMG-CoA) reductase which is overexpressed in colorectal cancer cells were associated with a $47 \%$ relative reduction in risk of CRC [16]. Whereas, NSAIDS, which are believed to act by inhibiting cyclooxygenase-2, which has been shown to be upregulated in CRC, was shown to yield an adjusted relative risk of 0.5 (95\% confidence interval of $0.4-0.7)$ [17].

Boundless insight into the complex exchange between the individual and their environment and their associated risk of developing $\mathrm{CRC}$ has been gained by the sheer depth of epidemiological studies conducted to date [18-22]. However, the above findings should be interpreted with caution due to the dangers of inferring causation from association. Nevertheless, the above studies provide a promising 
foundation from which the topic of my dissertation, i.e. the use of Anthos as both a chemopreventive and chemotherapeutic agent, has been constructed.

\section{Role of carcinogens}

A variety of environmental carcinogens such as PAHs, PCBs and PCDDs [18] have been linked to the development of CRC. One specific class of carcinogens that are particularly ubiquitous and relevant though are polycyclic aromatic hydrocarbons (PAHs). The PAH, B[a]P, is a byproduct of incomplete combustion of organic materials and is commonly found in charbroiled meat, vehicle exhaust, coal tar, and tobacco smoke. In addition to these more well-known sources of exposure, $\mathrm{B}[\mathrm{a}] \mathrm{P}$ has been found to contaminate a wide array of foods including cooked meat (4 $\mathrm{ng} \mathrm{B}[\mathrm{a}] \mathrm{P} / \mathrm{g}$, i.e., $4 \mathrm{ppb}$ of cooked meat), as well as kale and collard greens $(0.5 \mathrm{ng} \mathrm{B}[\mathrm{a}] \mathrm{P} / \mathrm{g})$ [19], with the daily estimated oral intake of $\mathrm{B}[\mathrm{a}] \mathrm{P}$ of 40.1 to $60 \mathrm{ng}$ for most individuals in a study comprised of residents of the metropolitan area of Washington, DC, USA [19]. It should be noted that the range of estimated $B[a] P$ intake for this study went up to $140-160 \mathrm{ng} / \mathrm{day}$ [19]. The classical mechanism by which $\mathrm{B}[\mathrm{a}] \mathrm{P}$ causes carcinogenesis is by intercalating into DNA and ultimately covalently binding of its electrophilic metabolites with guanine bases. The most active metabolite of $\mathrm{B}[a] \mathrm{P}$, benzo[a]pyrene-7,8diolepoxide (BPDE) is classified as a group 1 carcinogen by the International Agency for Research on Cancer (IARC) and is the result of the bioactivation of $\mathrm{B}[\mathrm{a}] \mathrm{P}$ by enzymes including the cytochrome P450 (CYP) 1A1 and/or $1 \mathrm{~B} 1$ and microsomal epoxide hydrolase $(\mathrm{mEH})(1,4,5)$. It should be noted that various enzymes including members of the glutathione s-transferase (GST), uridine 5'- 
diphospho-glucuronosyltransferase (UGT) and sulfotransferase (SULT) families are involved in the detoxification of the intermediates along this bioactivation pathway. Intercalation of DNA acts to distort the structure of DNA disrupting the copying of DNA, which in turn causes mutations. BPDE has also been found to target p53 thereby altering the tumor suppression of cells which may ultimately lead to cancer [23].

\section{Role of bacterial dysbiosis}

Although bacteria have long been associated with a variety of diseases such as diphtheria, tetanus, tuberculosis, typhoid fever and botulism, to name a few, only recently has the role of bacteria in carcinogenesis been elucidated. In fact, bacteria such as Heliobacter pylori and Enterotoxigenic Bacteroides fragilis (ETBF) have been linked to a variety of cancers including gastric and colon cancer, respectively. One particular toxin producing bacteria, ETBF has recently come to the forefront in its role in colon cancer. Although the gram negative $B$. fragilis bacteria make up a minority when compared to the prevalence of other bacterial community members, they cause the majority of anaerobe-associated invasive disease cases [24]. A particularly toxic member of the $B$. fragilis family is ETBF. This bacteria secretes the enterotoxin $b$. fragilis toxin (BFT) which is a 20 $\mathrm{kDa}$ zinc-dependent metalloprotease toxin which indirectly stimulates the cleavage of E-cadherin thus leading to increased colonic permeability [25]. Additionally, it leads to activation of $\beta$-catenin, which further leads to enhanced proliferation of cancerous CECs [26]. ETBF induces Stat3/Th17 inflammation which further fuels colon tumorigenesis [27]. Interestingly, the BFT toxin, of which 
three similarly acting isotypes, BFT-1, BFT-2, and BFT-3, have been identified, is structurally similar to other bacterial toxins including the tetanus and diphtheria toxins [24]. Recent work from the Sears lab shows that patients with the inherited disease, familial adenomatous polyposis (FAP), which is caused by as APC mutation, are more prone to having patchy bacterial biofilms that were composed primarily of Escherichia coli (colibactin producing) and ETBF [28]. Thus, given the clinical relevance and pathogenicity, ETBF bacteria is an ideal candidate for further research.

\section{Outline of mutations linked with development of CRC}

In addition to the extrinsic influences discussed above, another key participant in the dialogue of colorectal carcinogenesis is genetics. Some of the key genes that have been found to influence the development of CRC include TP53, KRAS/BRAF [29, 30], EGFR/PTEN [31-33], SMAD7 [34], MLH1 [35], MUTYH [36] and the adenomatous polyposis coli (APC) gene [37-40].

Both sporadic and hereditary cases of CRC have been attributed to mutations in the adenomatous polyposis coli (APC) gene. It has been noted that $34-70 \%$ of sporadic colon cancer cases feature inactivating mutations in APC particularly in the mutation cluster region, with genetic aberrations such as deletions, insertions, point and missense mutations detected in the mutation cluster region in $72 \%$ of the patients studied [37]. It has also been shown that individuals with APC gene mutations possess a significantly increased risk for CRC (pooled OR of 1.42 and $95 \% \mathrm{Cl}$ of $1.16-1.74$ with a $\mathrm{P}$ value of 0.00085 [ [38]. Furthermore, it should be noted that APC mutations appear to be more likely to occur with TP53 
mutations (OR $3.513,95 \% \mathrm{Cl}$ of $1.212-10.184)$ and KRAS mutations (OR 1.995, $95 \% \mathrm{Cl}$ of $0.607-6.554)$ [40].

The important role that the above mutations, especially APC, play in the development of $\mathrm{CRC}$ has been historically well documented. Moving forward, the characterization or "fingerprinting" of an individual's specific mutations will be key in developing personalized therapeutic regimens for each patient.

\section{Pathways of genomic instability that can lead to CRC}

It is commonly accepted knowledge that three main molecular pathways of genomic instability are involved in the development of CRC and include: the chromosomal instability pathway $(\mathrm{CIN})$, the mismatch repair-deficient or microsatellite instability pathway (MSI), and the CpG island methylator phenotype (CIMP) pathway.

The CIN pathway, which was originally elucidated by Fearon and Vogelstein in 1990 , is believed to be responsible for most cases of CRC and is observed in 65$70 \%$ of sporadic CRC's [41]. These cases feature characteristic loss of heterozygosity (LOH), sub-chromosomal genomic amplifications and widespread aneuploidy. In Fearon and Vogelstein's seminal 1990 article, they proposed a multi-step model for colorectal carcinogenesis [42] (Figure 2). The initiating event in adenoma formation is the inactivation of the Adenomatous Polyposis Coli (APC) tumor suppressor gene which is followed by activating mutations of KRAS. Additional mutations in TGF- $\beta$ [43], PIK3CA [44], and TP53 [45] pathways then drive the subsequent malignant transformation. It should be noted that although 
APC mutations serve as the initiating event in human and mouse models, mutational activation of KRAS is unable to initiate cancer in vivo and only when combined with mutant APC will mutant KRAS promote tumor progression, thus supporting Fearon's multi-step model [46].

The MSI pathway accounts for approximately $10-20 \%$ of all CRCs [47]. Although the MSI pathway was originally elucidated for individuals with familial Lynch syndrome, most CRCs with MSI are sporadic. In fact, only $3 \%$ of all CRCs come from Lynch syndrome families [48]. MSI tumors caused by Lynch syndrome are associated with germline mutations in DNA mismatch repair (MMR) genes and often have KRAS mutations, but never have BRAF mutations.

Lynch syndrome tumors also typically occur in younger individuals and typically have a better prognosis than non-MSI tumors [49]. Approximately $50 \%$ of sporadic cases of MSI have BRAF mutations and are associated with CIMP, unlike Lynch syndrome tumors. As can be expected, sporadic cases of MSIassociated CRCs typically occur in older individuals[48].

The CpG island methylator (CIMP) is observed in approximately $30 \%$ of CRC cases [50]. As its name implies, the CIMP pathway promotes epigenetic instability and enables sporadic cancers to methylate the promoter regions of key tumor suppressor genes thereby effectively inactivating their expression [51]. In fact it is present in all tumors with MLH1 aberrant methylation [52]. CIMP positive tumors typically exhibit specific clinicopathological features such as proximal location and tend to be more common in older women, are often MSI and poorly differentiated. They also tend to have a distinct molecular profile which features 
low TP53 and high BRAF mutation rates [53]. Furthermore, cases of colorectal cancer that develop via the CIMP pathway tend to develop from sessile serrated adenomas rather than adenomatous polyps [54].

\section{Current standard of care}

As with most cancers current treatment options for individuals diagnosed with CRC include one or more of the following options: surgery, radiation therapy, non-targeted chemotherapy, or targeted therapy.

According to the American Cancer Society, drugs currently approved by the U.S. FDA for the treatment of CRC include: capecitabine, fluorouracil (5-FU), irinotecan, oxaliplatin, and trifluridine/tipiracil. One or more of these chemotherapeutic drugs are most often given over the course of treatment with more common treatment regimens with these drugs including: 5-FU, 5-FU with a vitamin called leucovorin which improves the efficacy of 5-FU [55], capecitabine, 5-FU with leucovorin and oxaliplatin (FOLFOX), 5-FU with leucovorin and irinotecan (FOLFIRI), capecitabine with either irinotecan (XELIRI or CAPIRI) or oxaliplatin (XELOX or CAPEOX), and irinotecan alone. 


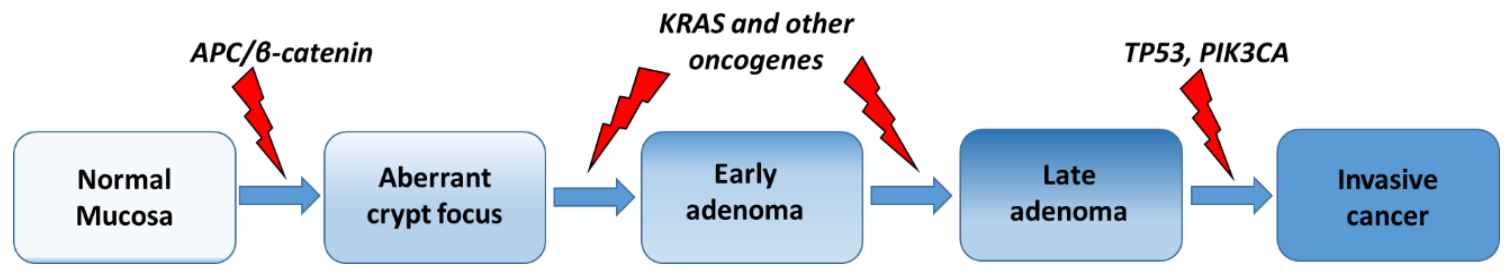

Figure 2: Adenoma-carcinoma sequence in the development of colorectal cancer 
A variety of side effects are associated with taking the standard chemotherapeutic drugs including: nausea, vomiting, fatigue, diarrhea, suppression of bone marrow, immunosuppression, and liver damage [56, 57]. Given the current limitations and these side effects associated with the current standard of care, additional treatment options are warranted.

In addition to non-targeted chemotherapeutic treatment options, targeted therapies are also common, including: bevacizumab, regorafenib, ramucirumab and ziv-aflibercept, which are antiangiogenesis therapies, and cetuximab and panitumumab which are anti- EGFR therapies [58]. Although, one of the key benefits of targeted therapy is the reduction in off-target side-effects, they are also accompanied with side-effects including gastrointestinal perforation, arterial thromboses which include strokes and myocardial infractions [59].

In addition to the above therapies, within the past year the FDA has approved use of the immune checkpoint, PD-1, inhibitor, pembrolizumab for the treatment of CRC cases that have mismatch repair deficient (dMMR) and microsatellite instability high (MSI-H) abnormalities. However, like the previously mentioned therapies, pembrolizumab treatment has been associated with immune related adverse effects such as inflammation of the lungs, colon, kidney, thyroid and pituitary gland [60]. Therefore, there remains significant room for the development of cost-effective, non-toxic prevention and treatment options.

Treatment options for individuals diagnosed with colon cancer are typically dependent upon their specific stage. Additional factors such as age and other health problems are also considered for the optimal treatment plan [61]. In most 
cases, when the cancer has not spread to distant sites, surgery is typically the first main treatment. For more advanced-stage cancers, an adjuvant chemotherapy may also be recommended. Adjuvant chemotherapy may be recommended as early as stage II, but is more typically recommended beginning in stage III [61]. For the more advanced stage IV cases of colon cancer where the disease has spread to distant organs and tissues, a key component of the treatment is chemotherapy since in most cases surgery will not cure these individuals. Since the early 2000's, 5 new drugs have been approved for the treatment of stage IV colon cancer. These drugs fall into one of the three following classes: multi-kinase inhibitors, anti-angiogenic (Bevacizumab, Zivaflibercept), or EGFR blockers (Cetuximab, panitumumab) [62].

Key limitations in the current standard of care include, but are not limited to the following areas: off-target side effects, the current gap that exists in adjuvant therapy for stage I and especially stage II cancers of the colon, and limitations in treatment options for older individuals. Given these limitations, research investigating alternative preventative treatment options is of great importance.

\section{Familial Adenomatous Polyposis (FAP)}

The majority of CRC cases originate from previously benign adenomatous polyps. This process of transformation from benign polyp to malignancy typically takes decades to occur, with approximately $85 \%$ of colorectal cases occurring after the age of 55 years, according to data acquired by the U.S. preventive services task force. A small subset of CRC cases stem from familial syndromes such as Lynch syndrome, FAP, attenuated FAP (AFAP), MUTYH-associated 
polyposis (MAP) and rare syndromes such as hyperplastic polyposis, PeutzJeghers syndrome (PJS) and juvenile polyposis syndrome [63, 64].

FAP is an autosomal dominant pre-cancerous colorectal condition with an incidence at birth of around 1/8,300 [65]. FAP occurs with equal frequency in both males and females and accounts for less than $1 \%$ of colorectal cases [65]. The disease may begin as early as the teenage years, with colon cancer developing on average by the age of 39 years old. Furthermore, approximately $7 \%$ of individuals with FAP will develop CRC by the time they reach 21 years old and $95 \%$ of FAP sufferers will develop CRC by the time they reach the age of 50 [66]. FAP is caused by mutation(s) in the APC gene. The APC gene, which is located on chromosome $5 q 21$ is a tumor suppressor gene that encodes for a 312 $\mathrm{kDa}$ protein with 2843 amino acids. It is expressed in a variety of fetal and adult tissues including colorectal epithelium as well as mammary [67].

Mutation(s) in the APC gene, $60 \%$ of which are nonsense mutations, have been shown to accelerate the initiation of the adenoma-carcinoma sequence since the inactivation of APC serves as the initial step in the development of CRC in FAP [65]. The APC protein serves as a scaffolding protein which affects cell adhesion and migration. The mammalian form of APC contains multiple binding domains including an oligomerization domain, an armadillo repeat domain, a repeat domain consisting of between $15-20$ residues which is important for binding to $\beta$ catenin, SAMP repeats which are important for axin binding, a basic domain that is responsible for microtubule binding, and finally $\mathrm{C}$-terminal domains, which bind the proteins, EB1 and HDLG [68]. 
APC interacts with a variety of other key proteins such as the Wnt/Wg pathway members, $\beta$-catenin and axin, the Rac guanine-nucleotide-exchange factor (GEF) Asef1, the cytoskeletal regulators EB1 and IQGAP1, as well as microtubules [69]. Mutations in APC lead to the accumulation of $\beta$-catenin in the cytoplasm where it binds and activates the T-cell factor (TCF) and lymphoid enhancer factor (LEF) families of transcription factors altering the expression of genes that encode for the metalloprotease matrilysin, ephrins, cyclin D1 and the proto-oncogene c-myc which in turn lead to changes in cellular proliferation, differentiation and migration [70].

If left untreated, individuals with FAP will develop CRC by their forties [71]. As discussed above, APC plays a key role in the multi-step model of colorectal cancer proposed by Fearon and Vogelstein (Figure 1). The disease is characterized by the development of hundreds to thousands of adenomatous colon polyps. Currently, the only approved primary modality of treatment for FAP is prophylactic cancer-preventive colorectal surgery. Given the lack of noninvasive treatment options and the role of FAP in CRC development, additional treatment options are of great need.

\section{Anthos: a promising treatment for FAP and colorectal cancer}

Several plant-derived compounds such as quinine, codeine, paclitaxel, apomorphine, have been an invaluable source of medicines for humans throughout history. One particular family of compounds that has sparked recent interest is flavonoids and members such as anthocyanins. 


\section{Flavonoids}

One particular class of relevance is the flavonoids which is one of the largest groups of secondary metabolites with well over 6000 molecules [72]. Compounds classified as flavonoids, which all share a common core 15-carbon skeleton, can be divided into 6 major classes: flavanols, flavonols, flavonones, flavones, isoflavones and anthocyanins. Flavonoids are commonly synthesized by plants in order to fulfil a variety of protective functions ranging from chromatic attractants for pollinators, deterrents for pests, protectants against UV-B, as well as antimicrobial agents [73].

\section{Anthocyanins and anthocyanidins}

Found in dark-colored vegetables, fruits, grains and flowers, anthocyanins, which comes from the Greek words Anthos for flower and kyanos for blue, provide the characteristic red, purple and blue hues to eggplant, blueberry, black rice and black sesame seeds. Over 600 structurally distinct anthocyanins have been identified in nature $[74,75]$. It should be noted that anthocyanins are known as anthocyanidins when non-glycosylated. Of the seventeen anthocyanidins found in nature, only six, including the glycosylated forms of cyanidin (Cy), delphinidin $(\mathrm{Dp})$, petunidin $(\mathrm{Pt})$, peonidin $(\mathrm{Pn})$, pelargonidin $(\mathrm{Pg})$ and malvidin $(\mathrm{Mv})$, are widely distributed [76]. The above anthocyanidins are typically bonded to glucose, galactose, arabinose, rhamnose, and xylose in either a mono-, di- or trisaccharide form. An additional layer of complexity is provided by the fact that the sugar moieties can also be acylated by various aromatic or aliphatic acids. The various combinations/permutations of anthocyanidins and their pendant sugars 
vary from plant to plant. Importantly, it should be highlighted that the active component of the compounds is the central anthocyanidin moiety.

The core structure of anthocyanidins (Figure 3 ) is composed of an aromatic ring A that is bonded to a heterocyclic ring $C$ containing oxygen that is bound via carbon to a third aromatic ring B. The substituted flavonoids contain a flavylium cation, which due to its conjugated double bonds, absorbs light with a characteristic peak range between $500-550 \mathrm{~nm}$. The specific hue(s) associated with each anthocyanin depend upon the degree of hydroxylation and type/number of substitutions. A total of 17 anthocyanidins have been isolated to date [77].

The specific anthocyanin fingerprint varies between each type of fruit, vegetable, grain or flower. For example, fruits such as red currants and elderberry contain primarily cyanidin, whereas blueberry, bilberry and jamun (Indian black plum) contain 5 anthocyanidins - cyanidin, delphinidin, petunidin, peonidin and malvidin, all in their glycosylated forms. Bilberry in particular contains a native mixture of delphinidin, cyanidin, malvidin, peonidin, and petunidin in a relative ratio of 33:28:16:16:7 [78]. As noted above, in addition to variations in the core anthocyanidin structure, variations in the pendant sugar moieties also varies from plant to plant. For example, even though red currant and blackcurrant are closely related, red currant contains mainly mono- and diglycosides, whereas black currant contains primarily rutinosides and only a minor glycoside component [79]. 


\section{Role of anthocyanins within plants}

Within plants, anthocyanins accumulate in vacuoles of a variety of cells in both vegetative as well as reproductive organs. Most of the 17 anthocyanidins isolated to date are found only in the reproductive structures of the plants whereas only six anthocyanidins (cyanidin, peonidin, delphinidin, petunidin, malvidin and pelargonidin) have been found in the vegetative organs [80].

Furthermore, anthocyanins are synthesized in the branch of the flavonoid biosynthetic pathway that is involved in the synthesis of isoflavonoids and flavonols [77]. Anthocyanins are thought to play a variety of different functions within plants ranging from as antioxidants, sunscreens, and even as metalchelating agents and in delaying foliar senescence under conditions of mineral imbalance or macronutrient deficiency [77]. Given their beneficial roles in plants, one can easily hypothesize that benefits may be gained from their consumption by humans.

\section{Anthocyanin absorption, stability, and digestion}

Absorption of anthocyanidins and anthocyanins by gut epithelial cells occurs via different mechanisms due to the structural differences. Due to the hydrophilicity added by the sugar residues on anthocyanins they must utilize facilitated transport mechanisms whereas (the sugar-free) anthocyanidins are more hydrophobic and are able to be passively absorbed by gut epithelial cells [81]. In fact, the hydrophobicity of a compound, expressed in terms of its partition coefficient (log octanol/water) is often used as a predictive means to determine the ability of a compound to passively diffuse across a biological membrane. 
Interestingly, it has been found that nonenzymatic deglycosylation does not occur in the human body for polyphenols such as quercetin [82]. Additionally, only polyphenols with attached glucose, arabinose or xylose and not rhamnose moieties can serve as potential substrates for human $\beta$-glucosidases. Therefore, rhamnose moieties can only be cleaved once they reach the colon where they can serve as a substrate for bacterial $\alpha$-rhamosidases [81]. This limitation may have important ramifications for the activity of rhamnose-containing anthocyanins in diseases of the small intestine or in diseases where digestive transport time is expedited such as inflammatory bowel disease (IBD).

Another important topic that must be addressed in the discussion of anthocyanin metabolism is the role of deconjugation and reconjugation reactions. Although anthocyanins do not appear to undergo as extensive of metabolism as other flavonoids, it will be discussed for completion since it occurs and is a variable that must be considered [83]. Following hydrolysis, the free aglycones undergo metabolic processing in the form of methylation, sulfation, glucuronidation or a combination of the above reactions $[84,85]$. Based on the structural differences between anthocyanins versus anthocyanidins, the impact that conjugation has on the biological properties of the circulating metabolites is evident. 


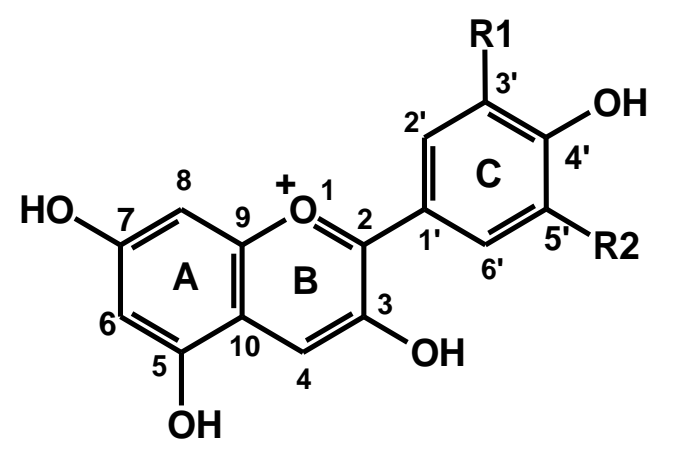

\begin{tabular}{l|l|l} 
& $\mathbf{R}_{\mathbf{1}}$ & $\mathbf{R}_{\mathbf{2}}$ \\
\hline Delphinidin & $-\mathrm{OH}$ & $-\mathrm{OH}$ \\
\hline Cyanidin & $-\mathrm{OH}$ & $-\mathrm{H}$ \\
\hline Peonidin & $-\mathrm{OCH}_{3}$ & $-\mathrm{H}$ \\
\hline Petunidin & $-\mathrm{OH}$ & $-\mathrm{OCH}_{3}$ \\
\hline Malvidin & $-\mathrm{OCH}_{3}$ & $-\mathrm{OCH}_{3}$ \\
\hline
\end{tabular}

Figure 3: Structures for the five anthocyanidins (the active moiety of anthocyanins) contained in blueberry and bilberry 
Overall, one should understand that a competition between the uptake of anthocyanidins and the excretion of more hydrophilic anthocyanins as well as their conjugated counterparts exists. Therefore, one could posit that by administering anthocyanidins either in their pure form or by nano-delivery systems to enhance oral bioavailability, one would enhance their therapeutic potential in the clinical setting.

\section{Data from In vitro work}

A variety of in vitro studies have been conducted in order to investigate the impact that anthocyanins derived from various sources have on colon cancer cell lines. Berry extracts containing anthocyanins isolated from cowberry, strawberry, bilberry, and blueberry have been shown to inhibit the proliferation of the colon cancer cell line HCT116 [86]. Furthermore, anthocyanin rich extracts derived from fruits including bilberry (Vaccinium myrtillus L.), grape (Vitis vinifera), and chokeberry (Aronia meloncarpa E.) have been shown to inhibit the growth of the colon cancer cell line HT-29 cells but not the non-tumorigenic NCM460 colon cells [87].

A variety of potential mechanisms have been suggested in the literature. For instance, anthocyanins isolated from the grape, Vitis coignetiae Pulliat were shown to induce caspase-dependent apoptotic cell death, activate p38-MAPK and suppress Akt in HCT-116 colon cancer cells [88]. Moreover, pelargonidin, found in a variety of berries such as strawberry and raspberry, was shown to inhibit proliferation of the colon cancer cell line HT-29 and induce apoptosis and G2/M cell cycle arrest by the intrinsic apoptotic pathway [89]. Additionally, 
delphinidin, found in fruits like blueberry, has also been shown to induce apoptosis and G2/M cell cycle arrest in HCT116 colon cancer cells [90]. The mechanism by which delphinidin led to G2/M arrest and apoptosis in HCT116 cells is believed to be due to suppression of the NF-KB pathway [90]. Anthocyanins and anthocyanidins have also been shown to induce significant oxidative DNA-strand cleavage in the presence of dithiotheritol (DTT), but not DMSO, which serves as a free radical scavenger, at physiological $\mathrm{pH}$. It was further hypothesized that at $\mathrm{pH} 7.5$, the quinones formed from anthocyanins and anthocyanidins autoxidize to yield hydrogen peroxide [91]. $\mathrm{Fe}^{3+}$ is believed to increase anthocyanin-induced cleavage since when it is reduced it is able to stimulate the production of hydroxyl radicals from hydrogen peroxide. The resulting hydroxyl radical, rather than hydrogen peroxide, is believed to induce the cleavage of DNA [91]. Only at high concentrations were anthocyanins also shown to inhibit topoisomerase relaxation [91].

\section{Data from in vivo studies}

The beneficial effects of anthocyanins shown in vitro have also been shown to exist in vivo. For instance, the anthocyanin-rich soybean fraction containing cyanidin-3-glucoside, delphinidin-3-glucoside and petunidin-3-glucoside, was

shown to significantly decrease the number of intestinal tumors in $\mathrm{Apc}^{\mathrm{Min} /+}$ mice fed a $0.5 \%$ anthocyanin-rich extract compared to control animals [92]. Furthermore, mucosal expression of cytosolic phospholipase $A_{2}$ and cyclooxygenase-2, which are both involved in inflammatory response, were significantly decreased in mice treated with $0.5 \%$ anthocyanin-rich extract [92]. 
Additionally, an in vivo study in $\mathrm{Apc}^{\mathrm{Min} /+}$ mice using $0.3 \%$ oenocyanin, which contains the 3-glucosides of delphinidin, cyanidin, petunidin, peonidin, and malvidin extracted from grape pomace, showed a reduction in adenoma burden and reduction in proliferation index in colonic adenomatous crypts [93]. A reduction in the expression of Akt, which is involved in a variety of cellular processes including glucose metabolism, apoptosis, cellular proliferation, transcription and migration, was also observed in $0.3 \%$ oenocyanin-treated mice [93]. Interestingly, oenocyanin anthocyanins and their metabolites were not found in the plasma but rather only in the urine and intestine [93]. Moreover, in a mouse model of colitis, dextran sulfate sodium (DSS) induced histological damage and weight loss were significantly improved in mice administered a red raspberry anthocyanin-rich fraction [94]. In the same study, anti-inflammatory properties were shown in vivo with overall reductions in the expression of cyclooxygenase-2 (COX-2), interleukin-1 beta (IL-1ß), IL-6 and inducible nitric oxide (iNOS) in red raspberry anthocyanin-rich fraction treated RAW264.7 cells [94].

A study assessing the impact of dietary cyanidin-3-glucoside and an anthocyanin mixture from bilberry on the development of adenomas in an $\mathrm{Apc}^{\mathrm{Min} /+}$ mouse model for FAP [95] showed that ingestion of cyanidin-3-glucoside or a bilberry derived anthocyanin mixture led to a reduction in adenoma load in a dosedependent fashion. It should be noted that although significant reductions in adenoma numbers were seen for cyanidin-3-glucoside $(p<0.001)$ and Mirtoselect ${ }^{\circledR}$ bilberry anthocyanins $(p<0.05)$, the doses that were used were $0.3 \%$ of the diet which is equivalent to $450 \mathrm{mg} / \mathrm{kg} /$ day or $2.6 \mathrm{~g} / 80 \mathrm{~kg}$ human dosage, 
when the authors extrapolated using the dose/surface area comparison between species [96]. When the Mirtoselect ${ }^{\circledR}$ native bilberry mixture was extrapolated back to the amount of fresh berries that would need to be consumed, it was concluded that an individual would need to consume $\sim 740 \mathrm{~g}$ of fresh bilberry a day [95]. Given the large nature of the dose used in the study, which is further complicated due to the presence of high sugar content (about $60 \%$ in blueberry), it is clear that one would need to lower the dose by identifying the active principles as well as eliminate large sugar content in order to be a clinically-viable option.

\section{Data from clinical trials}

A limited number of clinical trials have been undertaken to assess the impact that anthocyanins, taken in the form of either berry powder or as an anthocyanin-rich standardized bilberry extract, has on CRC. The first study was a clinical trial assessing whether an anthocyanin-rich standardized bilberry extract (Mirtocyan ${ }^{\circledR}$ ) administered to patients $(1.3,2.8$ or $5.6 \mathrm{~g}$, containing $0.5-2.0 \mathrm{~g}$ anthocyanins) daily for 7 days before surgery would induce pharmacodynamics changes that would be in agreement with chemopreventive efficacy [97]. Results from the study showed that a $7 \%$ decrease in proliferation was observed in tumor tissues taken from patients when compared to their pre-intervention values. Another clinical trial investigating the impact of freeze-dried blackberry powder (60 g/day, for 1 to 9 weeks) in colorectal cancer patients showed beneficial changes in GMCSF and IL-8, markers for proliferation and apoptosis respectively [98]. 
A clinical trial assessing the impact of black raspberry powder on biomarkers of colorectal tumor development showed that the methylation patterns of three Wnt inhibitors including WIF1, SFRP2, SFRP5 and the developmental regulator PAX6a were protectively modulated in normal and in colorectal tumor tissues in patients receiving the black raspberry powder $(60 \mathrm{~g} / \mathrm{d})$ for an average of 4 weeks. Furthermore, the protective modulation of methylation was associated with decreased expression of the enzyme that transfers methyl groups to the cytosine nucleotides in genomic DNA, DNA (Cytosine-5-)-Methyltransferase 1 (DNMT1) [99]. Interestingly, the authors also noted that black raspberry powder decreased $\beta$-catenin as well as protectively modified Ki-67, TUNEL, CD105, as well as DNMT1 in colorectal tissues from the 20 patients. CD105 and DNMT1 were also found to be modulated in adjacent normal tissues. The impact on $\beta$-catenin and E-cadherin expression was also shown to be dose dependent, with the high dose ( 4 weeks) black raspberry powder treatment yielding greater impact than the low dose ( 2 weeks) black raspberry powder treatment [99].

In addition to studies on CRC, anthocyanin-rich bilberry extract has also been shown to reduce the amount of pro-inflammatory cytokines including TNF- $\alpha$ and IFN- $y$ in colon biopsies from patients with ulcerative colitis (UC). Furthermore, serum levels from UC patients that were successfully treated with anthocyaninrich bilberry extract had enhanced levels of immunoregulatory IL-10 and the Th17 cell specific cytokine IL-22 and reduced levels of TNF- $\alpha$ and IFN-y [100]. In another clinical trial testing the impact of a standardized anthocyanin-rich bilberry extract on UC disease activity showed that after 6 weeks of treatment, $63.4 \%$ of 
patients achieved remission and $90.9 \%$ of patients showed a response. It was also noted that fecal calprotectin levels were also decreased during the treatment, which further suggests that intestinal inflammation was reduced [101].

It should be noted that all of the anthocyanin-mediated colorectal cancer clinical trials published to date have been conducted using anthocyanins rather than anthocyanidins. Although the bioavailability of anthocyanins is very low in the plasma $(<1 \%)$, greater bioavailability has been found in colonic tissues [102]. This suggests that they may have greater potential since they can directly interact with the colon tissue. Additionally, one can posit that additional benefits in uptake, if administered in the aglycone form (i.e., as anthocyanidins) or by utilizing a nano drug delivery vehicle such as bovine milk-derived exosomes (i.e. ExoAnthos).

\section{Exosomes}

\section{Size, history, sources}

Exosomes are defined as membrane-bound vesicles that range in size from 30$100 \mathrm{~nm}$, in diameter, feature a density in sucrose gradients of $1.13-1.19 \mathrm{~g} / \mathrm{ml}$, are highly enriched with tetraspanin molecules and are of endocytic origin [103]. Exosomes were discovered in 1983 by Pan and Johnstone. In 1989, Pan and Johnstone named the vesicles exosomes [104]. Since their identification, exosomes have been isolated from essentially all biological fluids such as saliva, peripheral blood, cerebrospinal fluid (CSF), urine, sweat, malignant effusions and milk [105]. 


\section{Exosome formation}

Exosomes are initially formed via a process of endocytosis, with the internalization of the cell membrane to yield endosomes (Figure 4). Following the formation of endosomes, many small vesicles are formed inside the endosome by invagination of parts of the endosomal, multi-vesicular body (MVB), membrane. The MVBs then fuse with the cell membrane thereby releasing the intraluminal vesicles into the extracellular space. Following their release, these vesicles are referred to as exosomes.

\section{Exosome composition}

Exosomal membranes are primarily composed of lipids and proteins and are enriched with lipid rafts [106]. The lipid composition of exosomes is distinctively rich in cholesterol, ceramide, sphingomyelin, and phosphatidylserine [107]. However, exosomes contain minimal to no lysobisphospatidic acid (LBPA), which distinguishes them from MVBs' intraluminal vesicles (ILVs) [107]. Furthermore, the fatty acids in exosomes tend to be saturated or monounsaturated, which in addition to the high concentration of cholesterol is believed to lead to the lateral segregation of these lipids into exosomes during their formation [108]. Results from proteomic studies suggest that exosomes contain specific proteins from endosomes, plasma membrane, and cytosol [109]. Examples of proteins from the endosome include Alix, Tsg101, Rab GTPase, SNAREs Annexin and Flotillin. 


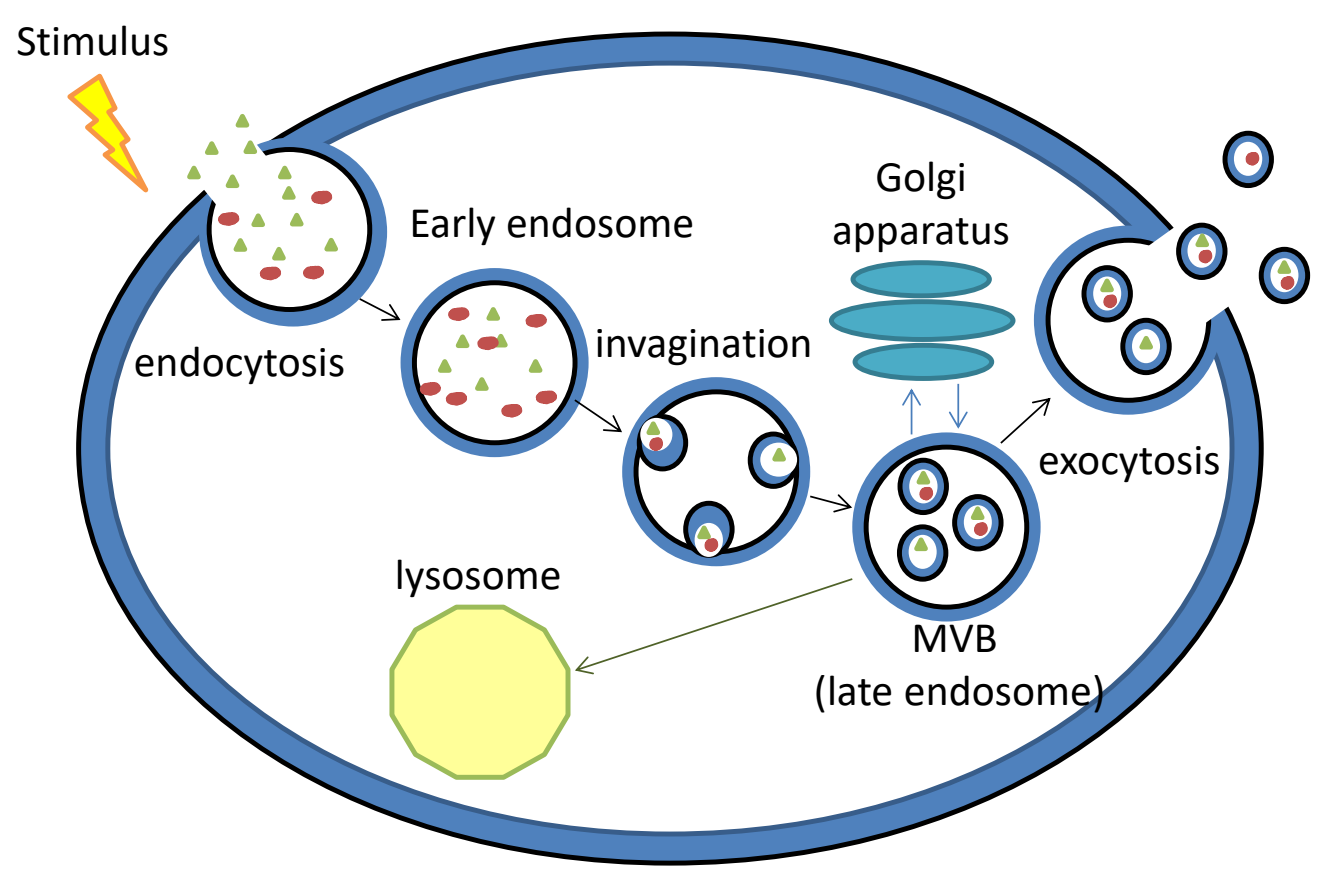

Figure 4: Classic pathway of exosome formation. After endocytosis, endosomes are formed via the internalization of the cell membrane (sidedness noted in the diagram as blue for the initial internal face and black for the initial external face). A process of invagination of the endosome yields smaller bodies inside the MVB, which can proceed to processing via the golgi apparatus, be released via exocytosis yielding exosomes or be degraded by the lysosome. 
Proteins originally associated with the plasma membrane include tetraspanins, such as CD63, CD81 and CD37. It should be noted that exosomes contain very few proteins from other intracellular organelles such as the nucleus, mitochondria and Golgi [107].

\section{Exosome cargo}

In addition to proteins, exosomes have been found to carry as cargo within their lumen, that include mRNA, miRNA, small noncoding RNA species such as structural RNAs, tRNA fragments, vault RNA, small interfering RNAs, RNA transcripts that overlap with protein coding regions and repeat sequences [108]. More recent data indicate the presence of some DNA as well [110]. The RNA cargo contained within exosomes can either be taken up by neighboring cells or transported by the circulating exosomes and taken up by distant cells. Ultimately, the RNA cargo in exosomes is believed to function as a form of genetic exchange between cells [106].

\section{Exosome role in cell to cell communication}

Once called the "garbage bag" of cells, exosomes have been found to play an important role in cell to cell communication. After finding that mRNAs and miRNAs were present in exosomes derived from mast cells, it was also found that the mRNA in these exosomes were transported to recipient cells and following uptake were translated into proteins thus providing evidence for the ability of exosomes to transfer of genetic material [111]. It was noted that not all mRNAs that were abundant in the secreting cells were packaged into exosomes 
thereby suggesting that specific targeting machinery for mRNAs may exist [111]. It has also been shown that tumor-derived exosomes can both suppress the function of immune cells by promoting the differentiation of regulatory $T$ lymphocytes or by inducing apoptosis of activated cytotoxic $T$ cells [112]. Exosomes can also induce immune responses by transferring tumor antigens to dendritic cells and to present tumor antigens [113]. Interestingly, it has been found that exosomes can fuse especially well with the plasma membranes under acidic conditions mimicking those of the cancer cell microenvironment [114].

\section{Exosomes as therapeutics}

Special interest in the application of exosomes for diagnostic as well as drug delivery purposes has recently taken root within the scientific community. A key area of research within our laboratory is in utilizing exosomes as a drug delivery vehicle. Prior to the work conducted in this laboratory the primary source of exosomes for drug delivery purposes was from cell culture media and serum. In order to overcome the obvious limitations posed by these sources such as quantity, safety, cost and translatability, our laboratory pioneered the use of exosomes derived from bovine milk $[115,116]$. Not only does bovine milk provide a scalable source of exosomes, it also exhibits cross-species tolerance. Furthermore, no adverse immune or inflammatory responses have been found [117].

Exosomes derived from raw bovine milk can effectively deliver a variety of both hydrophilic as well as lipophilic compounds such as curcumin, withaferin A (WFA), paclitaxel, celastrol and doxorubicin. Moreover, tumor targeting of the 
exosomes with folic acid was shown to increase biological efficacy. Exosomal formulations containing WFA, celastrol, or curcumin have shown increased efficacy over drug alone in both in vitro and in vivo against lung and breast cancer $[115,116]$. Prior to the body of studies contained within this dissertation no experiments were undertaken with colon cancer. In addition to the role of the exosomes as a drug delivery vehicle, they are also believed to deliver an additional "payload" of their own. For instance, our laboratory has identified the presence of immune-related miRNAs such as miR-146a, $-155,-181 \mathrm{a}$ and -223 in bovine milk derived exosomes [115].

\section{Overall Goals of Doctoral Research Project}

The overarching goal of my doctoral work has been to investigate the use of bilberry- derived Anthos as both a chemopreventive and chemotherapeutic agent in bacterially-induced CRC and to study how Anthos modulate the influence of the environmental carcinogen $\mathrm{B}[\mathrm{a}] \mathrm{P}$ on the balance between phase I and II enzymes. In order to accomplish this goal, my research project was divided into three specific aims, the interwoven nature of which is highlighted in each of the subsequent chapters:

Aim 1: Determine anti-proliferative efficacy of optimal mixture of Anthos against colon cancer and normal cells in vitro.

Aim 2: Determine efficacy of Anthos against colorectal cancer using a bacteriadriven $\mathrm{Apc}^{\mathrm{Min} /+}$ ETBF mouse model. 
Aim 3: Determine the mechanistic influence of Anthos on ETBF and B[a]P in CRC.

This dissertation has been written with the goal of submitting the findings for publication in peer reviewed journals. Thus, chapters two to four are intended to be able to exist independently, with the introductory and summary chapters aiming at forming the foundation for the subsequent chapters with the summary chapter tying together all of the mechanistic findings. 
CHAPTER 2:

NOVEL INSIGHT INTO THE PREVENTION AND TREATMENT OF COLORECTAL CANCER AND FAMILIAL ADENOMATOUS POLYPOSIS BY ANTHOCYANIDINS

\section{Introduction}

\section{CRC}

Colorectal cancer (CRC) is the third leading type of cancer diagnosed and third leading cause of cancer-related deaths within the United States each year. According to the American Cancer Society, an estimated 140,250 individuals will be diagnosed and 50,630 will die from CRC in the U.S. in 2018 alone. Although the overall incidence of developing CRC has been decreasing since the mid to late 1980s for individuals over 55 years old, a recent study has uncovered a disquieting increase in the CRC incidence for individuals below 55 years old [12]. Although much progress has been made in combating the disease due to advancements made in early detection of CRC, clinically-effective chemopreventive measures are still of great need.

\section{Familial adenomatous polyposis (FAP)}

The majority of CRC cases originate from previously benign adenomatous polyps. This process of transformation from benign polyp to malignancy typically 
takes decades to occur, with approximately $85 \%$ of CRC cases occurring after the age of 55 years, according to data acquired by the U.S. preventive services task force. A small subset of CRC cases stem from familial syndromes. One such precondition for colorectal cancer is FAP. FAP is an autosomal dominant precancerous colorectal condition with an incidence at birth of around 1/8,300 [65] that is caused by mutation(s) in the adenomatous polyposis coli (APC) gene. FAP occurs with equal frequency in both males and females [65]. The disease may begin as early as the teenage years, with colon cancer developing on average by the age of 39 years. Furthermore, approximately $7 \%$ of individuals with FAP will develop CRC by the time they reach 21 years and $95 \%$ of FAP sufferers will develop CRC by the time they reach the age of 50 [66]. The only currently approved standard of care for the treatment of FAP is surgical resection of the colon [118]. Even after removal of the colon, individuals with FAP are still at risk for developing adenomas at other sites [118]. Given the limited treatment options for FAP and need for clinically- effective chemopreventive measures for $\mathrm{CRC}$, there is a need for additional research for better treatment and prevention methods.

\section{Previous work in chemoprevention of FAP and CRC}

A wide variety of compounds have been considered in both pre-clinical and clinical populations for the prevention of CRC and/or FAP. Clinical trials conducted with chemopreventive agents for CRC include non-steroidal antiinflammatory drugs such as aspirin [119] and cyclo-oxygenase-2 (COX-2) inhibitors, calcium [120], vitamin D [121], folic acid [122], and dietary phenolics 
such as resveratrol [123], curcumin [124], green tea extract [125], soy isoflavones [126], black raspberry powder [99], pomegranate extract [127] and bilberry extract [97]. However, the application of these interventions either were deemed ineffective or required large dosages $[128,129]$. Therefore, identification of plant bioactives present in the human environment for improved clinical translation is of utmost importance.

\section{Anthocyanins/Anthocyanidins}

Throughout history, plants have served as an invaluable source of medicines including but not limited to paclitaxel, quinine, and digoxin to name a few. One particular class of compounds, the anthocyanins, have recently been of great interest in the scientific and medical community due to their reported therapeutic activities including anti-inflammatory [94], chemopreventive and chemotherapeutic [86, 130], obesity control, cardiovascular disease prevention, and alleviation of the symptoms of diabetes [131]. Anthocyanins, which come from the Greek words Anthos for flower and kyanos for blue, are ubiquitous plant pigments that provide the characteristic red, purple and blue hues to vegetables (eggplant, purple cabbage), fruits (berries), grains (black rice, purple corn) and flowers (hibiscus). In fact, over 600 anthocyanins have been identified at the current time. Anthocyanins feature an aryl-substituted chroman ring system. Differences between each specific anthocyanin is dependent upon functionalization group(s) at the 3 and 5 prime locations and the composition of the pendant sugars. Furthermore, the active moiety of anthocyanins, 
anthocyanidins are devoid of the sugar residues but feature the same key central ring structure and various permutations of hydroxyl, hydrogen and methoxy functional groups (Chapter 1, Figure 3).

Studies from our own laboratory as well as others have shown therapeutic activity of anthocyanins against a variety of cancers including but not limited to breast, lung, esophageal, skin and CRC [130,132]. One such study by Cooke et al. [95] showed that ingestion of cyanidin-3-glucoside or a bilberry-derived anthocyanin mixture showed a modest reduction in adenoma in CRC in a dosedependent fashion. This, however, required large doses (450 mg/kg/day) which would translate to $2.6 \mathrm{~g} / 80 \mathrm{~kg}$ person. When the native bilberry extract (Mirtoselect) was extrapolated to the amount of fresh berries that would need to be consumed, it was concluded that an individual would need to consume $\sim 740 \mathrm{~g}$ of fresh bilberry a day. Given the large nature of the dose used in the study, which is further complicated by the presence of high sugar content (about $60 \%$ ), it is clear that one would need to lower the dose by identifying the active principles for clinical translatability. Use of berry extracts can reduce doses and elimination of the sugars would overcome these concerns. Previous work from our group has shown that anthocyanidins yield greater potency in vitro and in vivo against lung cancer $[78,133]$. The goal of the series of experiments contained within this chapter was to elucidate whether Anthos would be an efficacious treatment in preventing and treating FAP and a bacterially-induced model of CRC. 


\section{Materials and methods}

\section{Anthocyanins and anthocyanidins}

The anthocyanin, delphinidin 3,5-diglucoside was a kind gift from Dr. Inder P. Singh of the National Institute of Pharmaceutical Education and Research (S. A. S. Nagar, India). The individual anthocyanidins, cyanidin, delphindin, petunidin and malvidin were purchased from Chromadex (Irvine, CA). The native mixture of anthocyanidins (or Anthos) isolated from standardized bilberry extract, with purity of $>80 \%$ was generously provided by $3 \mathrm{P}$ Biotechnologies, Inc. (Louisville, KY).

\section{Isolation of bilberry-derived Anthos}

The native bilberry Anthos was further enriched using C18 Sep-Pak cartridges (Waters, Milford, MA). Anthos were eluted in acidified $(0.1 \% \mathrm{HCl})$ ethanol. The enriched extract was then dried using a Savant SC210A Speed-Vac (ThermoFisher Scientific, Waltham, MA) and stored under argon at $-20^{\circ} \mathrm{C}$. Purity was verified using HPLC-PDA-UV. Briefly, $15 \mu \mathrm{l}$ samples were analyzed using a Shimadzu Premier C18 reverse-phase column (250x4.6 mm i.d., $5 \mu \mathrm{m})$. Mobile phase A was composed of water: formic acid: acetonitrile (87:10:3) and mobile phase B was composed of water: formic acid: acetonitrile (40:10:50). The flow rate was $0.6 \mathrm{ml} / \mathrm{min}$ and the gradient condition was $0-5 \min 5 \% \mathrm{~B} ; 5-15 \min 15 \%$ B; $15-20$ min $25 \%$ B; $20-30$ min 35\% B; $30-40$ min $45 \%$ B; $40-45 \min 100 \%$ B; 45-50 min 5\% B. Detection of Anthos was at $520 \mathrm{~nm}$ by PDA-UV and total Anthos concentration was calculated using a standard curve. The reference 
compounds were purchased from Chromadex (Irvine, CA) and Cayman Chemical Company (Ann Arbor, MI).

\section{Cells, culture conditions and treatments}

The APC wild-type HCT 116 (ATCC® CCL-247 ${ }^{\mathrm{TM}}$ ) colon cancer cell line and CCD-18Co colon (ATCC $\AA$ CRL-1459 ${ }^{\mathrm{TM}}$ ) normal cells were acquired from American Type Culture Collection (Manassas, VA). The APC mutant colon cancer cell line HT-29 (ATCCB HTB-38D ${ }^{\mathrm{TM}}$ ) was a kind gift from Dr. Nobuyuki Matoba (University of Louisville). HCT-116 and HT-29 cells were maintained in McCoy's 5A medium (Gibco, Grand Island, NY) supplemented with 10\% FBS, $100 \mathrm{U} / \mathrm{ml}$ penicillin and $100 \mu \mathrm{g} / \mathrm{ml}$ streptomycin in a humidified atmosphere containing $5 \% \mathrm{CO}_{2}$ at $37^{\circ} \mathrm{C}$. CCD-18Co cells were maintained in MeMa (Gibco, Grand Island, NY) supplemented with 20\% FBS, $100 \mathrm{U} / \mathrm{ml}$ penicillin and 100 $\mu \mathrm{g} / \mathrm{ml}$ streptomycin in a humidified atmosphere containing $5 \% \mathrm{CO}_{2}$ at $37^{\circ} \mathrm{C}$.

\section{Measurement of cell viability}

The cytotoxicity of individual anthocyanins and anthocyanidins and the Anthos in colon cancer and normal cell lines was assessed by enzymatic reduction of the tetrazolium dye MTT. Briefly, $3.0 \times 10^{3}$ cells/well were grown in a 96-well tissue culture plates and were then exposed to varying concentrations of test agents or vehicle for $24 \mathrm{~h}$ after seeding. After $72 \mathrm{~h}$ treatment, cells were incubated with 5 $\mathrm{mg} / \mathrm{ml}$ MTT reagent for $2 \mathrm{~h}$. The resulting formazan crystals were then solubilized in dimethyl sulfoxide and spectrophotometrically measured at $570 \mathrm{~nm}$ (Bio-rad, 
Philadelphia, PA). $\mathrm{IC}_{50}$ values were then determined using Calcysyn software version 2.1 (Biosoft, Cambridge, England).

\section{Western-blot analysis}

For western-blot analysis, $50 \mu \mathrm{g}$ of protein from in vitro and in vivo tissue lysates was resolved using gel electrophoresis and electrotransferred to polyvinylidene difluoride membranes by semi-dry transfer (Biorad Trans-blot SD, Hercules, CA). Blots were blocked with $4 \%$ dry powder milk or BSA for $1 \mathrm{~h}$ and then incubated with primary antibodies for p-EGFR (Y845), p-EGFR (Y1068), p-EGFR (Y1173), total EGFR, p-Src (Y418), total Src, pSTAT3 (Y705), total STAT3, pSTAT5 (Y694/Y699), total STAT5, COX-2, Cyclin D1, and Cyclin D2 which were all acquired from Santa Cruz Biotech (Santa Cruz, CA) at $4^{\circ} \mathrm{C}$ overnight and secondary antibodies (Santa Cruz Biotech, Santa Cruz, CA). conjugated to peroxidase for $1 \mathrm{~h}$ at room temperature. Blots were then developed with an ECL detection system. Densitometric analysis was then performed using ImageJ 1.x software [134].

\section{Animal model for FAP and CRC}

$\mathrm{Apc}^{\mathrm{Min} /+}$ mice are a well-established and accepted model for studying FAP and CRC. The mice characteristically exhibit a germline nonsense mutation at codon 850 of the APC gene that causes the spontaneous development of polyps, which predominantly occur in the small intestine by the age of $10-12$ weeks. 


\section{ETBF}

Enterotoxigenic Bacteriodes fragilis (ETBF) exists asymptomatically in $12.4 \%$ of individuals overall and in $27 \%$ of individuals with diarrhea symptoms [135]. Furthermore, presence of ETBF in the gut is a well-known global cause of diarrheal disease that is accompanied by colitis in both humans and animals. The pathogenicity associated with ETBF is due to the secretion of a $20 \mathrm{kDa}$ zincdependent metalloprotease toxin, $B$. fragilis toxin (BFT), which binds to colonic epithelial cells and leads to the cleavage of the tumor suppressor protein, Ecadherin, and the secretion of interleukin-8 [136]. Overall, this process leads to the stimulation of proliferation and migration of human colon cancer cells [137]. BFT has also been shown to induce pro-inflammatory cytokine secretion by further activating the NFKB pathway [137].

\section{In vivo $\mathrm{FAP}$ and $\mathrm{CRC}$ studies}

Animal experiments were performed in agreement with an approved protocol by the Institutional Animal Care and Use Committee at the University of Louisville. Breeding colonies were established by Dr. Nejat K. Egilmez's lab [138] at the University of Louisville using C57BL/6J Min/+ $\left(\mathrm{Apc}^{\mathrm{Min} /+}\right)$ mice that were originally attained from Jackson Laboratories (Bar Harbour, ME). Mice were genotyped for the APC mutation using PCR according to the protocol established by Jackson Laboratories. Mice were fed a standard chow diet. Animals received diet and water ad libitum and were maintained on a standard light/dark cycle for the duration of the study. For the FAP study, treatments began when animals were 
8-9 weeks old. Male $(n=4)$ and female $(n=4) A c^{\mathrm{Min} /+}$ mice were administered by oral gavage $40 \mathrm{mg} / \mathrm{kg}$ Anthos or vehicle control. Animals were treated 3 times per week for 5 weeks. Animals were euthanized at 13-14 weeks. Following fixation of intestines using formalin, intestinal polyps were counted using a microscope (Leica EZ4, Wetzlar, Germany) by four different experienced individuals after blinding the samples.

Two CRC studies were performed to test the impact of the Anthos on tumor number. At 5-6 weeks of age, animals were administered antibiotic. Four days later the animals were administered ETBF to promote tumorigenesis and one week following ETBF inoculation, animals began their respective treatment regimen. In the first study male $\mathrm{Apc}^{\mathrm{Min} /+}$ mice were administered by gavage 20 $\mathrm{mg} / \mathrm{kg}$ Anthos or vehicle control. Animals were treated 5 times a week for 4 weeks. Animals were culled in the fed state at 12 weeks and colon tumors were counted. For the second study female $\mathrm{Apc}^{\mathrm{Min} /+}$ mice were administered by gavage $40 \mathrm{mg} / \mathrm{kg}$ Anthos or vehicle control. Animals were treated 3 times a week for 4 weeks. Animals were culled in the fed state at 11-12 weeks and colon tumors were counted.

\section{Data analysis}

Statistical analysis was performed using Graph Pad Prism statistical software version 4.03 (La Jolla, CA) and RStudio software version 1.0.153 (Boston, MA) Lattice package [139, 140]. Test for normality, F-test for equal variance, and 
appropriate t-test were used for both animal studies and for analysis of western blot results. $\mathrm{IC}_{50}$ values were determined using CalcuSyn software version 2.1 (Biosoft, Cambridge, England). Heat maps were constructed using RStudio software version 1.0.153 (Boston, MA) gplot package [140, 141].

\section{$\underline{\text { Results }}$}

\section{Anti-proliferative effects of Anthos on colon cancer cells but not normal colon cells}

Previous work within our laboratory has shown that anthocyanidins are more potent than their respective anthocyanins [133]. However, prior to pursuing work with Anthos, we first compared antiproliferative activity of anthocyanidins and anthocyanins by using two individual anthocyanins (anthocyanins delphinidin 3,5diglucoside, cyanidin 3,5-diglucoside and cyanidin 3-glucoside) with their respective anthocyanidins (delphinidin and cyanidin) in HCT-116 colon cancer cells. As shown in Figure 6, a clear benefit is derived for antiproliferative activity from the cleavage of the sugar moiety from the anthocyanin parent compound. This is best demonstrated by the reduction in the $\mathrm{IC}_{50}$ shown for delphinidin which yielded $>6$-fold reduction when the sugar moiety was absent. A reduction was also observed when comparing cyanidin versus cyanidin 3-glucoside, and cyanidin 3,5-diglucoside, with 2 - and nearly 5 -fold reductions, respectively (Figure 6). 


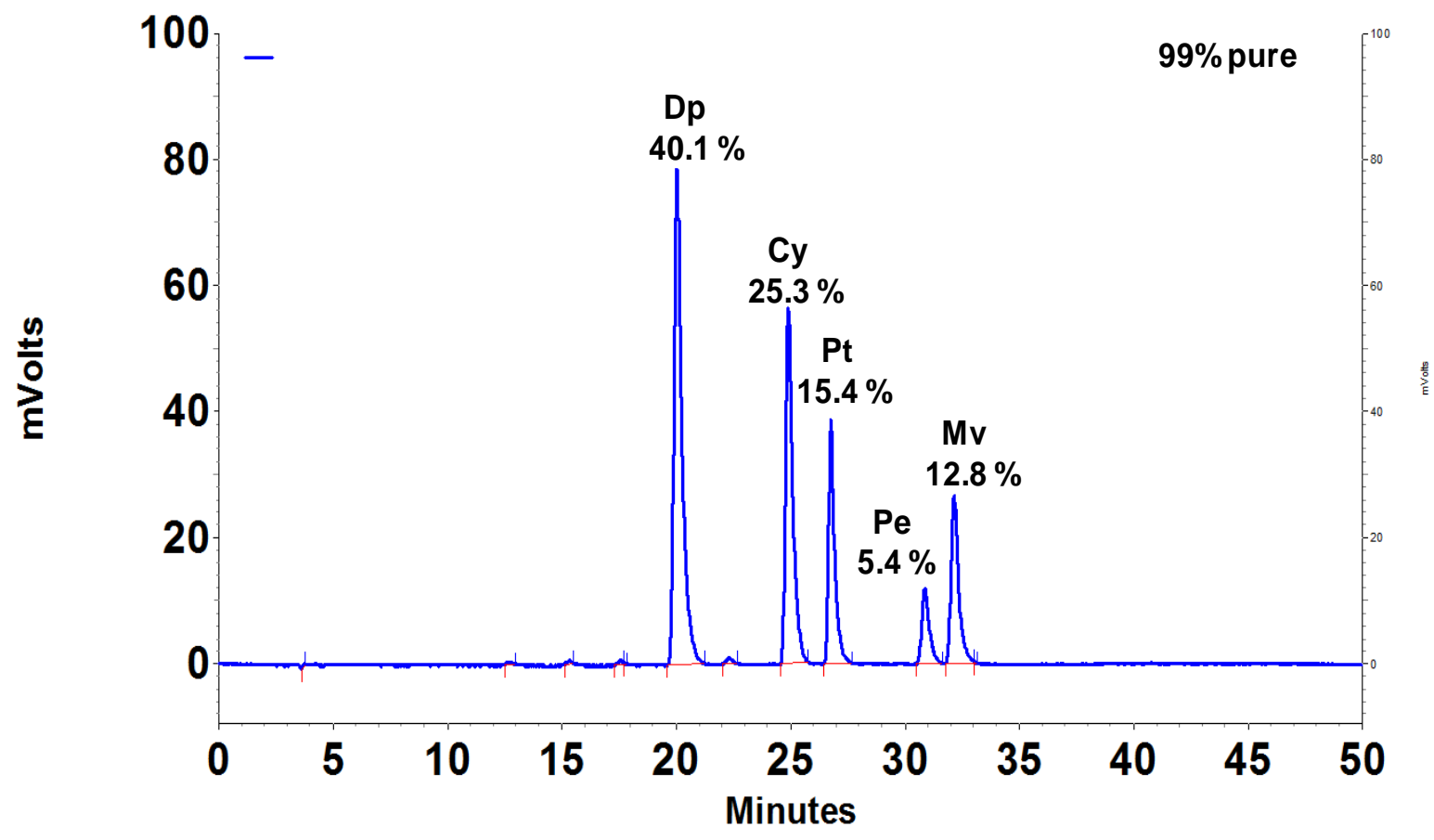

Figure 5. Representative chromatogram of C18-enriched bilberry-derived Anthos: HPLC profile of bilberry-derived anthocyanidin native mixture including delphinidin (Dp), cyanidin (Cy), petunidin (Pt), peonidin (Pe), and malvidin (Mv). Purity was verified to be $>96 \%$ for Anthos based on HPLC analysis. 

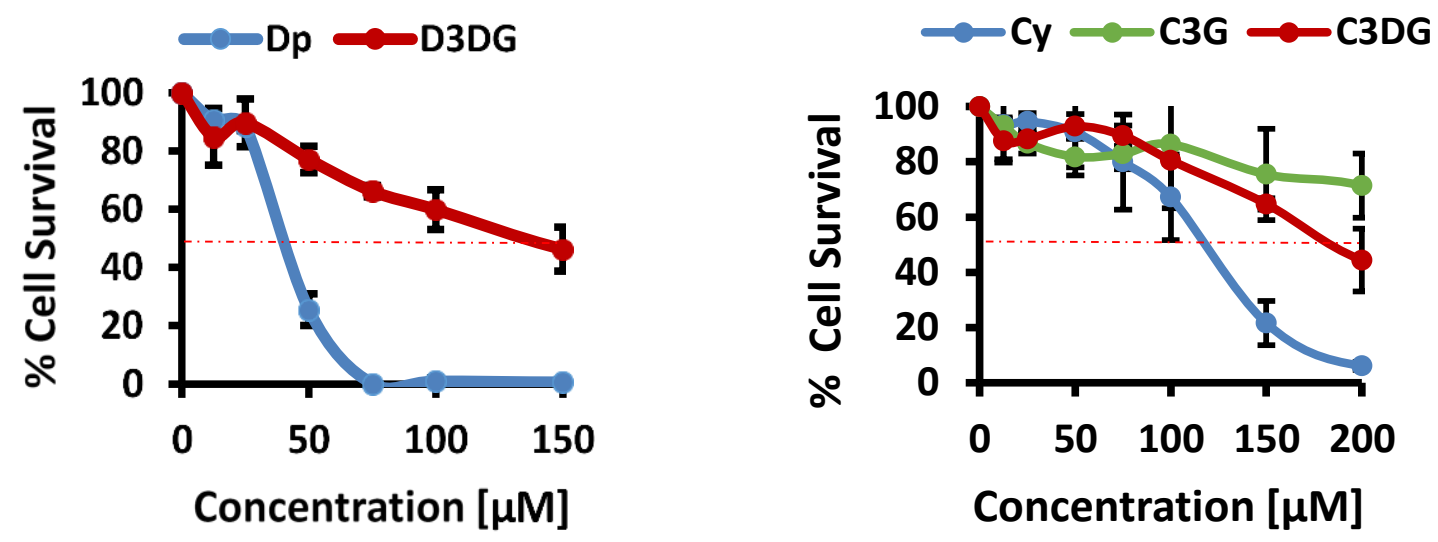

Figure 6. Antiproliferative activity of select anthocyanidins and their anthocyanin counterparts against colon cancer cell line HCT-116: Colon cancer HCT 116 cells were treated with various concentrations of the individual anthocyanidins, cyanidin (Cy) or delphinidin (Dp) and their anthocyanin counterparts cyanidin 3-glucoside (C3G), cyanidin 3,5-diglucoside (C3DG) or delphinidin 3,5-diglucoside (D3DG), respectively for $72 \mathrm{~h}$ and the effect on cell growth inhibition was assessed using an MTT assay. Data represent average \pm $\operatorname{SD}(n=3)$. 


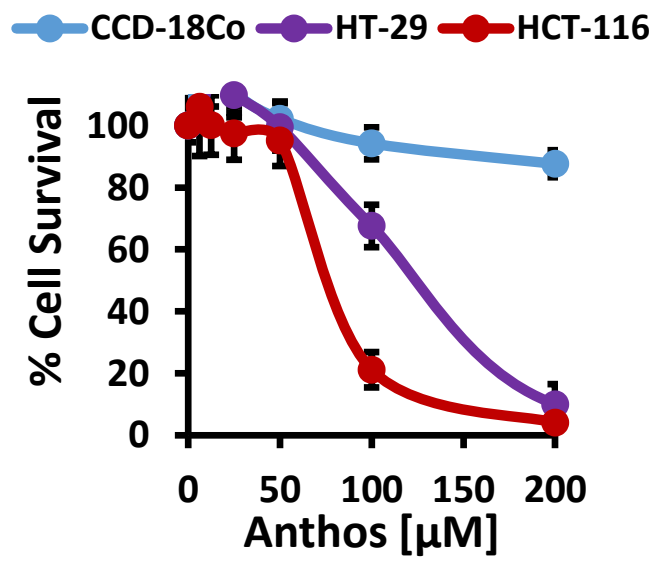

\begin{tabular}{|c|c|c|}
\hline Cell line & $\begin{array}{c}\mathrm{IC}_{50} \text { Anthos } \\
(\mu \mathrm{M})\end{array}$ & SI Anthos \\
\hline CCD-18Co & 1050 & - \\
\hline HCT116 & 75 & 14 \\
\hline HT-29 & 124 & 9 \\
\hline
\end{tabular}

Figure 7. Antiproliferative activity of Anthos against colon normal and cancer cells in vitro: Colon normal cells, CCD-18Co and colon cancer cells, HCT-116 and HT-29 were treated with various concentrations of Anthos for $72 \mathrm{~h}$ and the effect on cell growth inhibition was assessed using an MTT assay. Data represent average \pm SEM $(n=4)$. 
Once anthocyanidins were confirmed to yield greater antiproliferative properties than their parent anthocyanins, we assessed the antiproliferative properties of a native mixture of Anthos found in bilberries and whether this native mixture showed any in vitro toxicity toward normal colon CCD-18Co cells (Figure 7). Tabulated selectivity index (SI) values (Table 1) of 14 and 9 clearly show that Anthos selectively targets colon cancer cells (HCT-116 and HT-29) over the normal cells (CCD-18Co). It should also be highlighted that these values are well above the recommended minimal $\mathrm{SI}$ value of 3 that is commonly used to determine whether a drug selectively targets cancer cells over normal cells.

\section{Impact of Anthos on polyp development in $\mathrm{Apc}^{\mathrm{Min} /+}$ mice FAP model}

A wide variety of plant-derived compounds, including but not limited to compounds such as curcumin, epigallocatechin gallate and anthocyanins have been shown to have chemopreventive properties [129]. However, large doses have been a hallmark prerequisite for their efficacy in vivo [129]. Therefore, in order to test whether Anthos is both efficacious and translatable for the required dose to elicit such properties, we investigated the effect of the Anthos on intestinal polyp number utilizing $\mathrm{Apc}^{\mathrm{Min} /+}$ mice. Both male and female mice $(\mathrm{n}=4$ per group) were treated with $40 \mathrm{mg} / \mathrm{kg}$ Anthos three times per week (approximating $17 \mathrm{mg} / \mathrm{kg} / \mathrm{day}$ ) for four weeks by oral gavage. Results from the

study (Figure 8a) showed a greater than 2-fold overall reduction in intestinal polyps in Anthos versus vehicle control. The overall results in both male and female mice treated with the Anthos elicited polyp reductions that were 


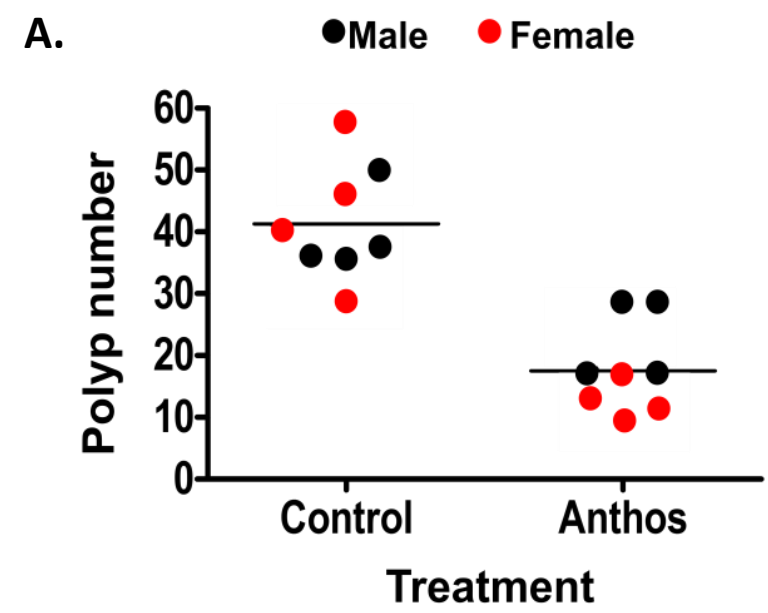

B.

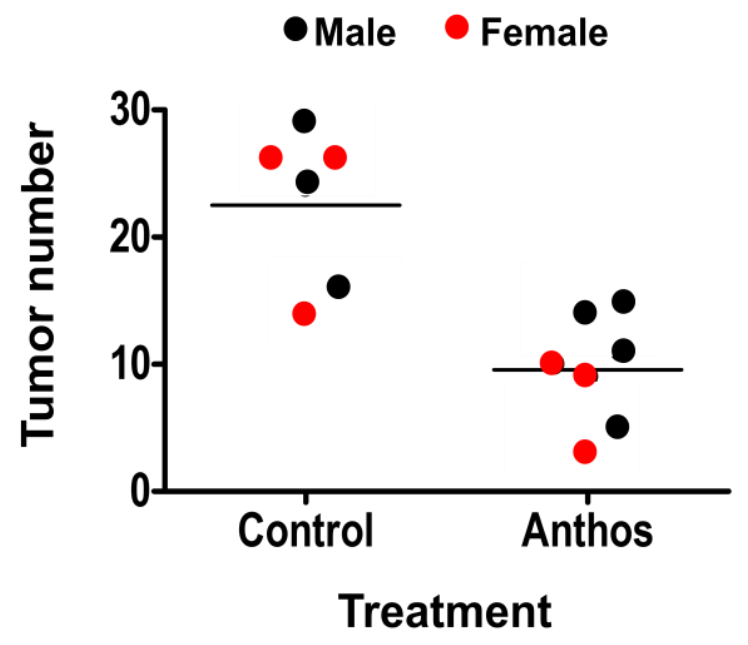

C.

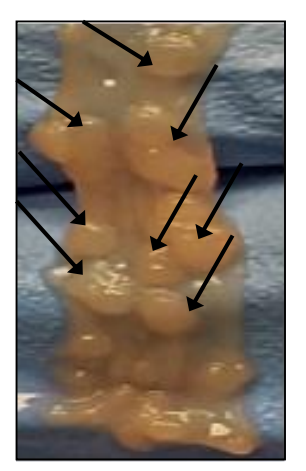

Control

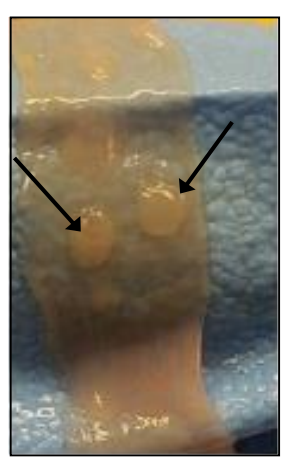

Anthos

Figure 8. Anti-polyp and anti-tumor activities of Anthos against intestinal polyps and colon tumors: a) $\mathrm{Apc}^{\mathrm{Min} /+}$ mice were treated via oral gavage with Anthos three times a week (40 mg/kg bw) or vehicle control. Data represent the distribution of animal polyp counts by gender, with the average noted. Male 
Anthos versus control $P=0.02$ and female Anthos versus control $P=0.004$. b) $\mathrm{Apc}^{\mathrm{Min} /+}$ mice inoculated with ETBF were treated via oral gavage with Anthos three times per week (40 mg/kg bw), five times per week (20 mg/kg bw), or vehicle control. Data represent the distribution of animal tumor counts by gender, with the average noted. Male and female Anthos versus control $P<0.001$. c) Representative images of colons taken from control versus Anthos-treated animals, with arrows highlighting some of the tumors. 
statistically significant ( $P=0.02$ and $P=0.004$, respectively). Interestingly, when the data was stratified by sex, it was noted that the effect of Anthos treatments was somewhat enhanced in female mice which showed 3.1 fold reductions in polyp numbers $(P=0.004)$ as compared to the 1.8 fold reduction $(P=0.02)$ found within males.

\section{Impact of Anthos on colon tumor development in ETBF Apc ${ }^{\mathrm{Min} /+}$ mice CRC} model

Previous work within our group has shown that Anthos decreases tumor burden in lung and ovarian cancer models [142] [78]. However, no work has been reported to assess the impact of bilberry-derived Anthos against either FAP, colorectal cancer or bacterially-induced cancers. Therefore, following the completion of in vitro testing of Anthos in both APC wild-type and APC mutant cell lines, we investigated whether Anthos treatment would impact colon tumor in a bacterially-driven $\mathrm{Apc}^{\mathrm{Min} /+}$ colorectal cancer mouse model. In these studies, mice which were treated with Anthos at either $20 \mathrm{mg} / \mathrm{kg}$ Anthos 5 times per week (i.e. $14 \mathrm{mg} / \mathrm{kg} /$ day) or $40 \mathrm{mg} / \mathrm{kg} 3$ times per week (i.e. $17 \mathrm{mg} / \mathrm{kg} /$ day) for 4 weeks by oral gavage. Results show an average reduction of 2.6 fold in the number of colorectal tumors in Anthos-treated mice compared with control (Figure 8b and 8c). This reduction was highly statistically significant $(P<0.001)$. 


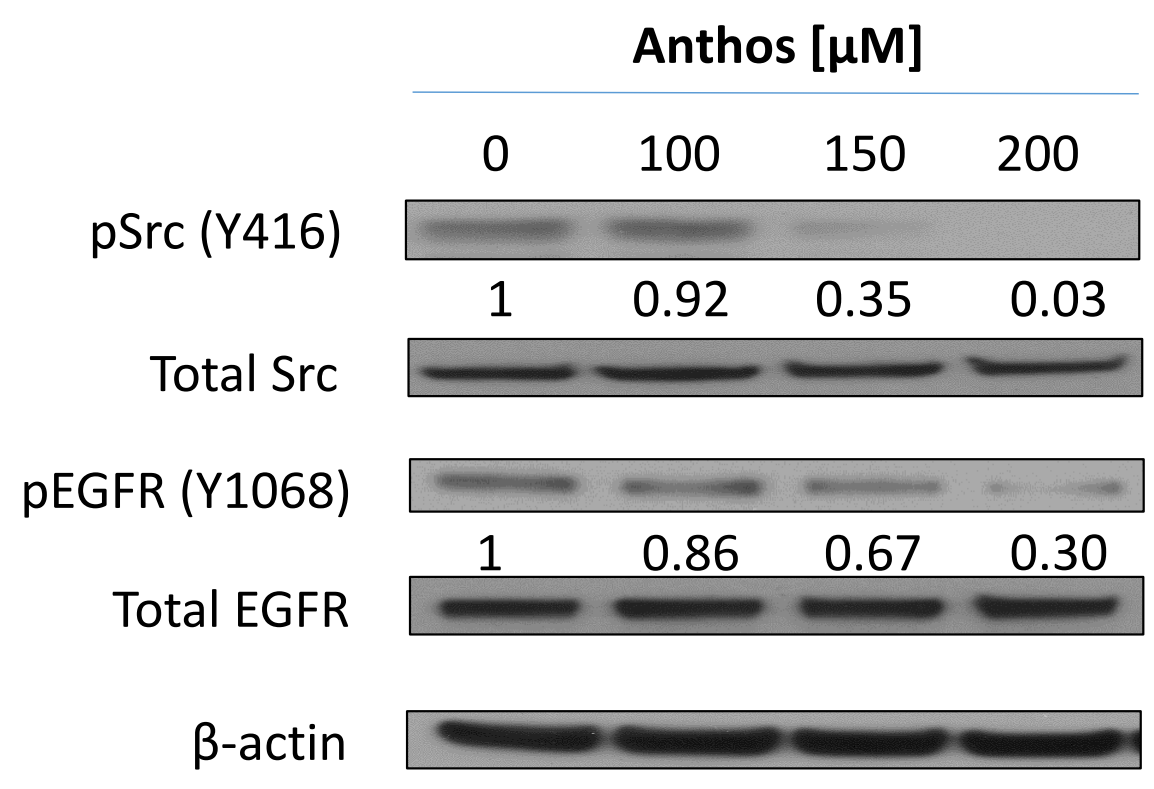

Figure 9. Changes in Src and EGFR phosphorylation after treatment with Anthos: Colon cancer cell line HT-29 was treated with various concentrations of Anthos overnight and the effect on phosphorylation of Src (Y418) and EGFR (Y1068) was assessed using western blot analysis and compared to total EGFR and $\beta$-actin loading control. Densitometry values listed are the ratio of each dose to the vehicle control for the phospho-protein/total protein, both corrected for $\beta$ actin loading control. 


\section{Insights into Anthos mechanism of action}

To understand Anthos mechanism of action we focused on the Src and EGFRrelated pathways. Our preliminary in vitro work with lysates prepared from Anthos-treated APC mutant HT-29 cells showed that Anthos treatment led to a dose-dependent decrease in the phosphorylation status of EGFR (Y1068) and Src (Y418) (Figure 9). With these promising in vitro findings we next sought to determine the influence of Anthos treatment on the Src and EGFR-related pathways in an ETBF-treated $\mathrm{Apc}^{\mathrm{Min} /+}$ mouse model which has been reported to have increased EGFR activity and c-Src expression in the adenoma and intestinal enterocyte tissue. Results from our analysis (Figure 9) of the phosphorylation status of the tyrosine kinase, Src at position Y418 showed a significant decrease in phosphorylation in Anthos-treated animals compared to control animals. The phosphorylation status of transphosphorylation site Y845 of the receptor tyrosine kinase (RTK), EGFR, a site noted for its activation by Src was also significantly decreased in adjacent normal and colon tumor samples taken from Anthos-treated animals over control animals. The transcription factor, STAT5a/b, which is immediately downstream of EGFR Y845, also showed a significant decrease in phosphorylation at tyrosine positions 694 and 699 in both adjacent normal and tumor tissue samples from Anthos treated animals. The ultimate expression of the downstream targets cyclin D1, cyclin D2 and COX-2 were also significantly downregulated in the Anthos group for both adjacent normal and tumor tissue samples (Figures 10-12). Interestingly, significant differences in the phosphorylation status of the autophosphorylation sites of 
Adjacent normal
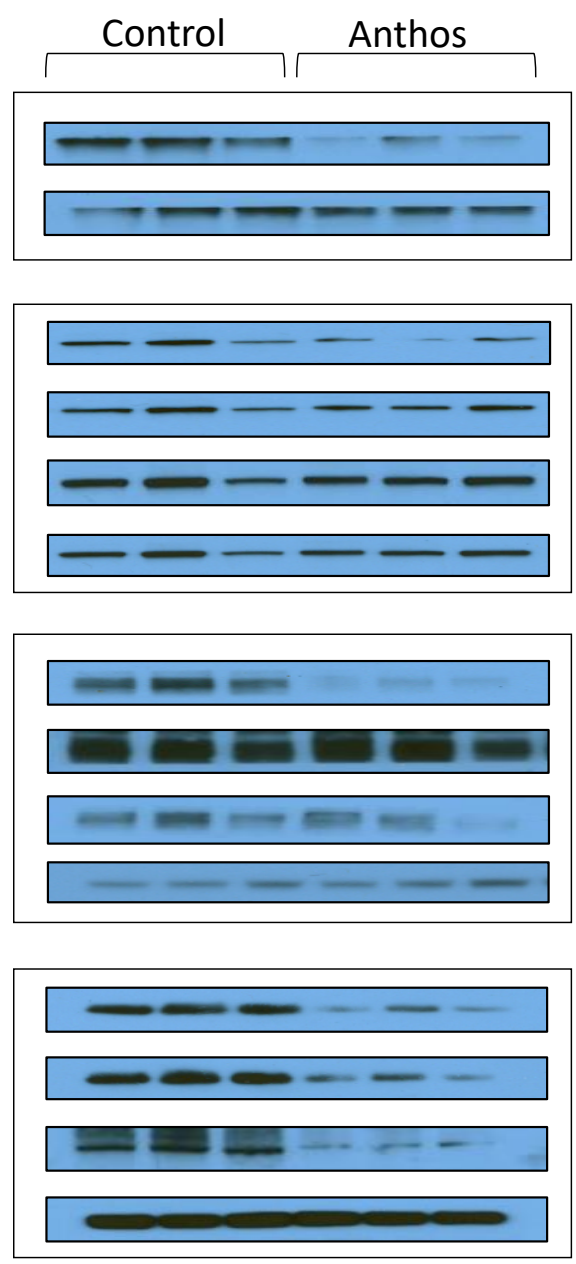

Tumor
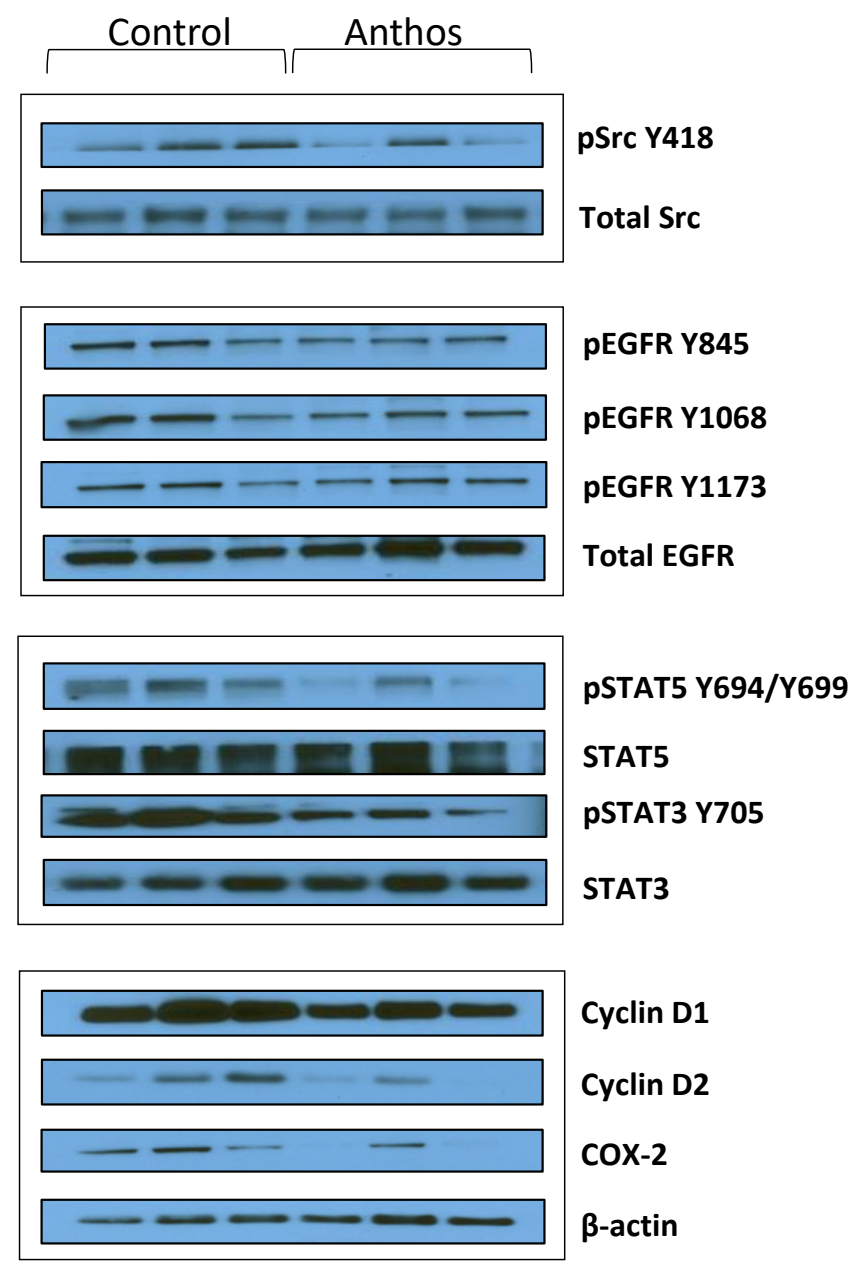

Figure 10. In vivo changes in Src and EGFR pathways following treatment with Anthos: changes in the phosphorylation and expression of Src and EGFR along with corresponding downstream pathway targets including STAT3, STAT5, cyclin D1, cyclin D2, and COX-2 in a) adjacent normal and b) tumor colon tissue taken from ETBF-inoculated $\mathrm{Apc}^{\mathrm{Min} /+}$ mice after treatment with Anthos or vehicle control as assessed using western blot analysis with $\beta$-actin loading control. Quantitative data summarized in Table 1. 


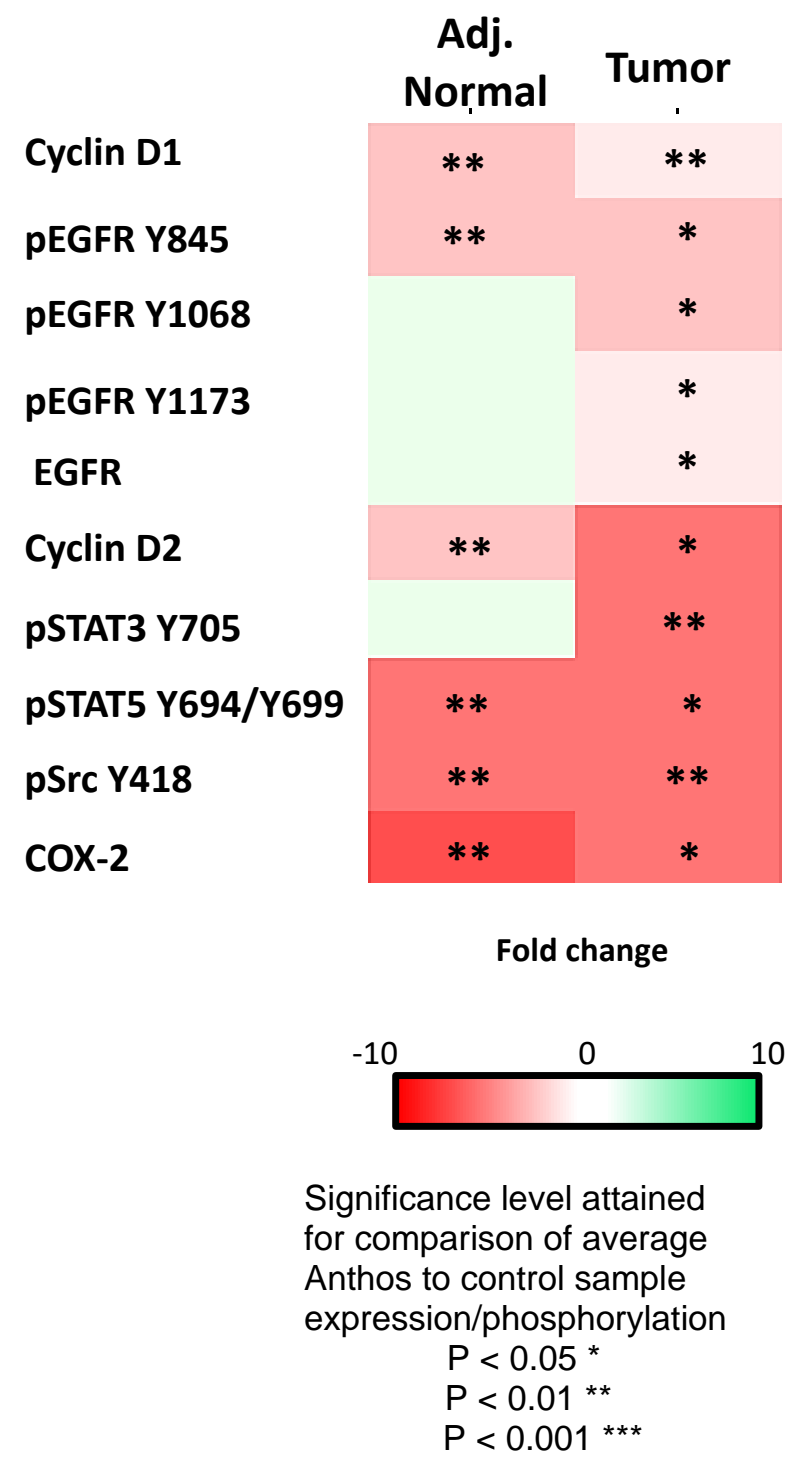

Figure 11. Representative heatmap for in vivo changes in Src and EGFR pathways following treatment with Anthos: representative heat map legend depicts the average fold change and significance level in either phosphorylation status or expression levels between Anthos treated and vehicle control animals within either adjacent normal $(n=3)$ or tumor colon tissue $(n=3)$. 
Table 1. Raw densitometry values calculated for Src/EGFR pathway western blot analysis

\begin{tabular}{|c|c|c|c|c|c|c|}
\hline \multirow{2}{*}{ Target } & \multicolumn{3}{|c|}{ Adjacent Normal } & \multicolumn{3}{c|}{ Tumor } \\
\cline { 2 - 7 } & Control & Anthos & Fold change & Control & Anthos & Fold change \\
\hline Src & 6883 & 7922 & 1 & 9558 & 8474 & -1 \\
\hline Src (Y418) & 20688 & 4816 & -4 & 20516 & 6259 & -3 \\
\hline EGFR & 18661 & 20531 & 1 & 18388 & 20599 & 1 \\
\hline pEGFR (Y845) & 18141 & 5950 & -3 & 17230 & 8659 & -2 \\
\hline pEGFR (Y1068) & 19771 & 17469 & -1 & 25990 & 12493 & -2 \\
\hline pEGFR (Y1173) & 21496 & 20029 & -1 & 16854 & 10731 & -2 \\
\hline pSTAT3 & 15938 & 11067 & -1 & 25354 & 5274 & -5 \\
\hline STAT3 & 5088 & 7000 & 1 & 10291 & 8208 & -1 \\
\hline pSTAT5 & 19969 & 3436 & -6 & 25057 & 6779 & -4 \\
\hline Cyclin D1 & 22846 & 7075 & -3 & 27696 & 14658 & -2 \\
\hline Cyclin D2 & 21036 & 6480 & -3 & 15580 & 3503 & -4 \\
\hline COX-2 & 19808 & 2687 & -7 & 18224 & 4358 & -4 \\
\hline
\end{tabular}




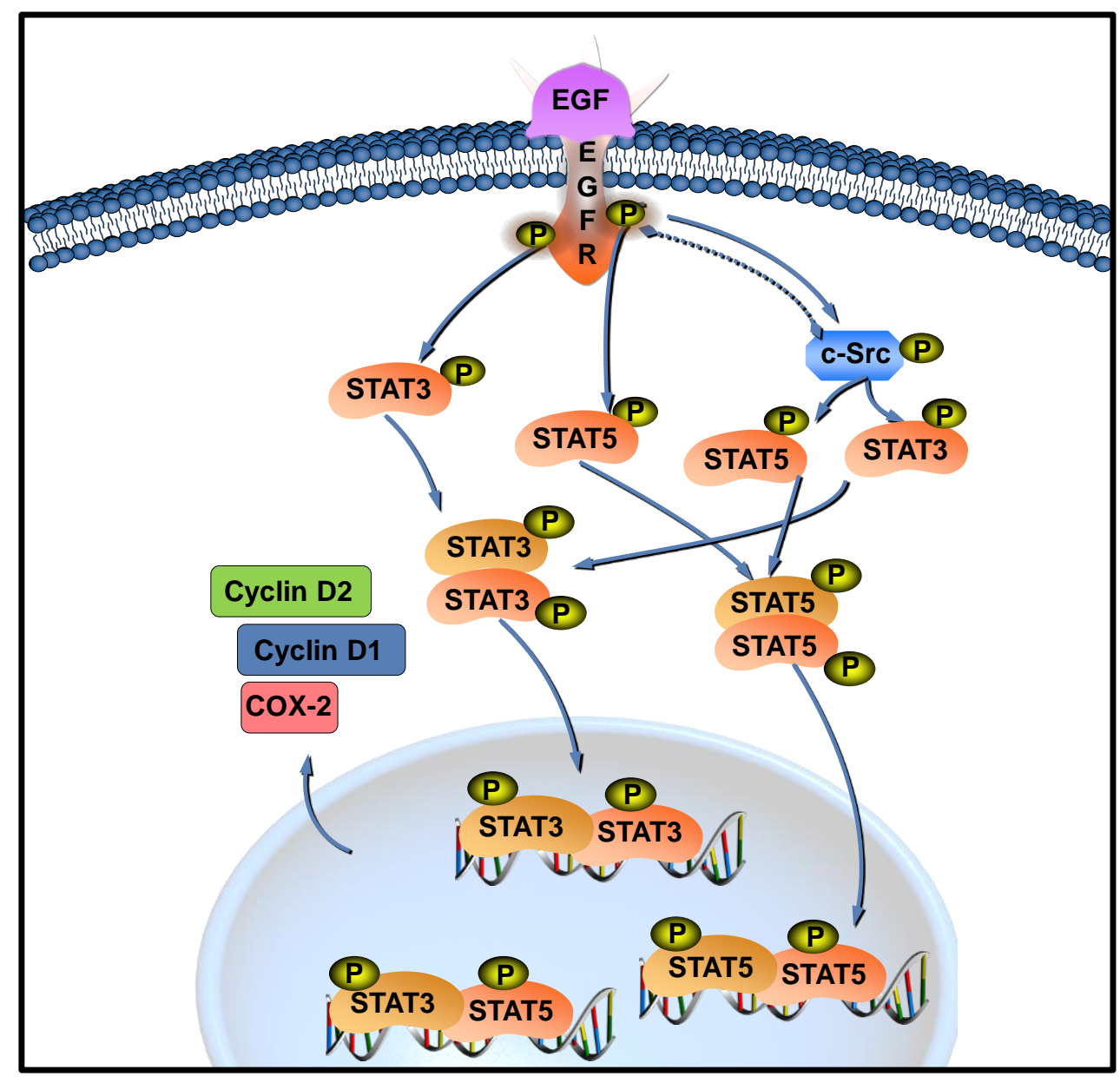

Figure 12. Pathway involving modulation of Src and EGFR phosphorylation status and downstream targets: the pathway deduced is based upon results from western blot analysis for adjacent normal and tumor tissue samples and is a modified version of the Qiagen EGFR signaling pathway. 
EGFR Y1068 and Y1173 were noted in the tumor but not the adjacent normal colon tissue of Anthos treated compared to control animals. This difference between adjacent normal and tumor tissue was also noted in the phosphorylation status of the immediate downstream protein STAT3, with a significant decrease in the phosphorylation at Y705 observed only in the tumor tissue and not the adjacent normal tissue in Anthos treated animals. A significant decrease in the levels of total EGFR, which was factored into the determination of phosphorylation status, was also seen in only the tumor samples of Anthos treated mice.

\section{Discussion}

Although much progress has been achieved in the treatment of CRC, it remains the third leading cause of cancer-related death in the U.S. Furthermore, FAP remains an orphan disease with the only viable treatment option being surgical resection of the colon. Even after this drastic procedure, individuals with FAP are still at a greater risk for developing cancers of the small intestine. With the above factors in mind, the development of alternative prevention and treatment strategies for combating both FAP and CRC are of great importance and represent an unmet need.

One particular class of plant bioactive compounds that have been studied for their health-promoting properties is that of the anthocyanins. This family of colored pigments is particularly promising given its long history as a dietary constituent for many humans. However, anthocyanins, when taken in their native 
form are often plagued by issues with their limited bioavailability and stability, which effectively limit their translation into a clinically-viable option due to the large doses that would need to be consumed in order to elicit therapeutic efficacy. With this in mind, the focus of this study has been to utilize the Anthos, which we have previously shown to be more active than the native anthocyanin counterpart in A549 lung cancer cells [133]. This relationship is also valid in colon cancer with delphinidin, and cyanidin yielding greater potency than their 3monoglucoside and 3,5-diglucoside anthocyanin counterparts. These results are not surprising considering that anthocyanidins are somewhat more lipophilic due to their higher partition coefficients than their anthocyanin counterparts. For example, the anthocyanin, cyanidin 3,5-O-diglucoside has an octanol to water (Kow) partition coefficient of 0.21 as opposed to its anthocyanidin counterpart, cyanidin which has a partition coefficient of 10.07 [143]. These higher partition coefficients enable anthocyanidins to be taken up passively by cells whereas anthocyanins are known to require active transport and thus may be limited in their transport efficiency/limitation [81].

Anthos were not only more potent than their anthocyanin counterparts, but exhibited selectively toxicity towards colon cancer cells over the normal colon cells with SI values that were well above the recommended cutoff for lack of toxicity. We had previously shown that Anthos were non-toxic in wild-type mice [144]. Furthermore, work with anthocyanins derived from blueberry, black currant as well as elderberry showed no toxicity when administered to rats (20 
$\mathrm{mg} / \mathrm{kg} /$ day), mice ( $25 \mathrm{mg} / \mathrm{kg} /$ day), or guinea pigs or rats ( $>3 \mathrm{~g} /$ day for 15 days or 90 days) [145].

Given the lack of more favorable/less invasive treatment options for FAP and the severity and ultimate lethality of the disease at early ages, there is a great need for developing alternative preventative treatment options. With this unmet need, the potential application of Anthos to the prevention of FAP is a key possibility based on our in vivo findings indicating significant decreases in intestinal polyp number with Anthos intervention in $\mathrm{Apc}^{\mathrm{Min} /+}$ mouse model. In addition to the promising results attained with the chemoprevention in the FAP study, Anthos exhibited significant anti-tumor activity against ETBF induced colorectal tumors in the $\mathrm{Apc}^{\mathrm{Min} /+}$ mouse model.

ETBF, a subtype of $B$. fragilis that secrets the metalloprotease enterotoxin $B$. Fragilis toxin (BFT), is associated with diarrheal disease in both humans and animals as well as active inflammatory bowel disease. A study looking at the prevalence of ETBF found that $26.8 \%$ of individuals with diarrhea and $12.4 \%$ of individuals without diarrhea had stool samples that were positive for the presence of ETBF [135]. Furthermore, a recent study looking at the bacterial composition in individuals with FAP compared to normal controls found that the colonic mucosa of individuals with FAP were highly enriched with genes for the toxin, $\mathrm{BFT}$, that is secreted by the ETBF bacteria [28]. In view of our encouraging findings, it is presumable that use of Anthos in human inflammatory bowel 
conditions and colorectal tumors associated with ETBF and FAP could be of great benefit.

We have previously shown that Anthos favorably modulate targets such as $\beta$ catenin, cyclin D1, cyclin B1, pERK, VEGF proteins, c-myc and MMP9 in lung cancer H1299 cells [25]. Furthermore, anthocyanin substitution patterns were found to lead to different cellular signaling cascades effects [36]. For instance, only malvidin which features methoxy groups at the $3^{\prime}$ and $5^{\prime}$ positions of the Bring lead to inhibition of cAMP-specific phosphodiesterases (PDEs) whereas, cyanidin and delphinidin were shown to inhibit EGFR in human vulva carcinoma A431 cells [36]. In this report, we deduced mechanistic work utilizing APC mutant colon cancer cells and adjacent normal and colon tumor tissue samples from ETBF Apc ${ }^{\mathrm{Min} /+}$ mouse model treated with Anthos. It was previously shown that APC deficiency was associated with an increase in EGFR activity and c-Src expression in $\mathrm{Apc}^{\mathrm{Min} /+}$ mouse adenomas and intestinal enterocytes [146]. Furthermore, In addition to increasing the secretion of chloride and permeability of intestinal epithelial cells, BFT also activates STAT3 and TH17 responses and leads to increased COX-2 [147]. However, no work had been conducted to assess the impact of Anthos or anthocyanins on Src in any cancer model or Anthos on EGFR in a colorectal cancer model. In vitro work using the APC mutant HT-29 colon cancer cells showed a clear dose-dependent decrease in the phosphorylation status of Src (Y418) and EGFR (Y1068). Results from our survey of the Src and EGFR-related pathways show a significant decrease in Src 
phosphorylation (Y418), EGFR phosphorylation (Y845) and the downstream mediator STAT5a/b (Y694 and Y699), both in adjacent normal and colon tumor tissue taken from ETBF treated $\mathrm{Apc}^{\mathrm{Min} /+}$ mice that received Anthos treatment. Furthermore, phosphorylation status of EGFR at Y1068 and Y1173 along with the downstream mediator STAT3 (Y705) was shown to decrease in tumor tissue in Anthos treated mice. Key regulators of cell cycle progression and inflammation, including Cyclin D1, Cyclin D2 and COX-2, were shown to be favorably modulated by Anthos, with the most dramatic reductions in expression observed in tumor tissue samples. Overexpression of COX-2 has been noted in colon tumor tissue and plays a role in the pathogenesis of FAP, ETBF and ultimately colon cancer [148, 149]. Overall, these findings provide novel mechanistic insight into the method by which Anthos achieve both chemopreventive and therapeutic effects against a highly relevant bacteriallydriven colon cancer model.

In summary, our data provide compelling evidence for the use of a bilberry Anthos against FAP and CRC. Anthos were shown to yield greater potency and efficacy than their counterpart anthocyanins and no toxicity toward normal colon cells in vitro. Furthermore, in vivo anti-polyp and anti-tumor efficacy along with corresponding mechanistic insight suggest that Anthos lead to a potent decrease in the phosphorylation status of Src, EGFR, STAT5, STAT3 and expression of key markers for proliferation and inflammation including cyclin D1, cyclin D2, and 
COX-2. Overall, results from this study offer an exciting possibility for potential treatments with berry Anthos for FAP and CRC in the future.

\section{Abbreviations}

CRC: colorectal cancer, Anthos: native anthocyanidins mixture from billberry, FAP: familial adenomatous polyposis, APC: adenomatous polyposis coli, COX-2: cyclo-oxygenase-2, Cy: cyanidin, Dp: delphinidin, Pt: petunidin, Mv: malvidin, Pe: peonidin, HPLC: high performance liquid chromatography, PDA: photodiode array detector, UV: ultraviolet, ETBF: enterotoxigenic Bacteriodes fragilis, BFT: B. fragilis toxin. 
CHAPTER 3:

THE ROLE OF ANTHOCYANIDINS IN RE-EQUILIBRATING METABOLIC SHIFTS INDUCED BY DYSBIOSIS OF THE GUT MICROBIOME AND THE UBIQUITOUS ENVIRONMENTAL CARCINOGEN, BENZO[a]PYRENE

\section{Colorectal cancer (CRC)}

Although much progress has been made in the diagnosis and treatment of cancer over the last thirty years, CRC remains as a looming threat on the horizon. For instance, although the incidence of $\mathrm{CRC}$ has been trending downward since the mid to late 1980s for individuals 55 years or older, a recent study has found a rather disconcerting uptick in the CRC incidence for individuals below 55 years old [12]. According to the CDC, the third most common form of cancer in the US is CRC. Furthermore, CRC is the third leading form attributed to cancer-related deaths each year. In fact, according to the American Cancer Society, it is estimated that 140,250 individuals will be diagnosed and 50,630 will die from CRC in the US in 2018 alone. Epidemiological studies suggest that lifestyles with diets rich in fat, smoking, and alcohol consumption as well as exposure to environmental pollutants and dysbiosis of gut microbiota increase the risk of developing $\mathrm{CRC}[3,150]$. Much progress has been made in combating the disease due to advancements made in early detection of CRC. However, options for chemoprevention from environmental insult and an understanding of 
how such treatments could alter the dialogue between one's microbiome and environmental toxins have yet to be achieved.

\section{Factors in the development of CRC}

\section{Environmental factors in CRC}

Environmental factors such as air pollution, cigarette smoke, and dietary contamination have been epidemiologically and mechanistically linked to an increased risk of CRC [151-153]. One particular class of environmental pollutants that is especially pervasive is polycyclic aromatic hydrocarbons (PAHs). One of the most ubiquitous members of this family is benzo[a]pyrene $(\mathrm{B}[\mathrm{a}] \mathrm{P})$ which is found in cigarette smoke, as a contaminant in many foods, car exhaust fumes, wood burning and coal tar. In order initiate the carcinogenic process, $\mathrm{B}[\mathrm{a}] \mathrm{P}$ undergoes bioactivation by enzymes, such as the cytochrome P450 (CYP) 1A1 and/or 1B1 and microsomal epoxide hydrolase $(\mathrm{mEH})(1,4,5)$, resulting in the formation of the ultimate carcinogen benzo[a]pyrene-7,8-diol-9,10-epoxide (BPDE). Various enzymes including members of the glutathione s-transferase (GST), uridine 5'-diphospho-glucuronosyltransferase (UGT) and sulfotransferase (SULT) families are involved in the detoxification of the intermediates along this bioactivation pathway (Figure 13). The metabolites of $\mathrm{B}[\mathrm{a}] \mathrm{P}$ are classified as group 1 carcinogens by the International Agency for Research on Cancer (IARC). BPDE intercalates DNA and ultimately covalently binds with guanine bases. This acts to distort the structure of DNA disrupting the copying of DNA, which in turn causes 


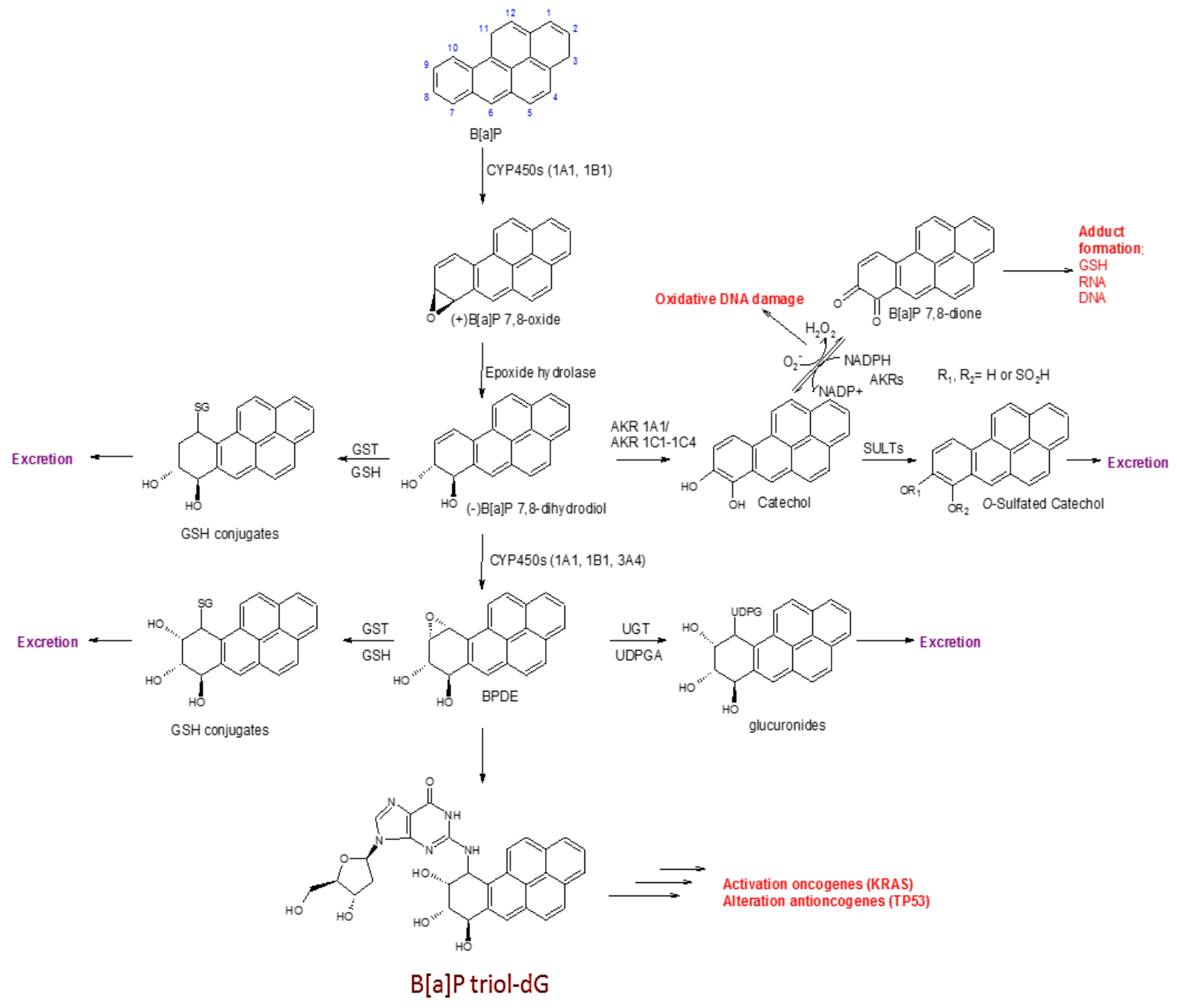

Figure 13. Metabolism of benzo[a]pyrene to carcinogenic BPDE vs. detoxification pathways 
mutations. BPDE has also been found to target p53 thereby altering the tumor suppression of cells which may ultimately lead to cancer [23]. Interestingly, research that measures all of the exposures of an individual in a lifetime and how

those exposures relate to health referred to as "exposome" has found that exposure to $B[a] P$ leads to the enhanced susceptibility of macrophage membranes to bacterial infection and ultimately may lead to immunosuppression [154]. Although one of the first carcinogens to be studied, $B[a] P$ remains a continued threat due to its widespread presence in the environment. Given the continued relevance of $\mathrm{B}[\mathrm{a}] \mathrm{P}$, work looking at how this and its metabolites interact with endogenous factors such as the gut microbiome is of high importance in addressing the management of several diseases including colon cancer.

\section{Bacterial carcinogens}

In addition to traditional carcinogens such as $\mathrm{B}[a] \mathrm{P}$, recent research has begun to uncover the importance of the gut microbiome in the development of CRC. Research has shown that imbalances in the intestinal microbiota lead to both an increase in inflammatory conditions as well as an increased production of carcinogenic metabolites, which may ultimately lead to neoplasia. Several bacteria have been associated with increased risk of developing CRC including: S. gallolyticus, H. pylori, virulent forms of Escherichia coli (E. coli), Fusobacterium nucleatum ( $F$. nucleatum), Salmonella enterica (S. enterica) and enterotoxigenic $B$. fragilis [155]. Furthermore, a bidirectional dialogue has been 
found to exist between the gut microbiome and environmental chemicals, with bacteria metabolizing the pollutants contributing to host toxicity and the contaminants altering the composition of gut microbiota [150]. This dynamic interaction between the host microbiome and environmental carcinogens is becoming ever more prevalent and relevant in the modern era. Furthermore, little is known regarding the impact that gut bacteria such as ETBF has on the expression of phase $\mathrm{I} / \mathrm{Il}$ enzymes. With this in mind, identifying a chemopreventive method to combat this omnipresent insult is of great importance.

\section{Anthos background}

\section{Anthos role in detoxification}

Several plant bioactives have been an invaluable source of medicines for humans. The family of plant pigments, known as the anthocyanins, have been identified with a variety of health benefits including chemopreventive and therapeutic effects due to their roles as anti-inflammatory, antioxidant agents and modulators of cytochrome P450 enzymes, CYP1A1 and CYP1B1 in mammary tissue [94, 131]. Found in dark-colored vegetables, fruits, grains and flowers, anthocyanins provide the characteristic red, purple and blue hues. Anthocyanins are, in part, converted to anthocyanidins (Anthos), the aglycone moieties, and, in fact, have higher antiproliferative and anti-inflammatory activities than the anthocyanins presumably due to higher cell uptake. 
The berry bioactive, Anthos present a promising chemopreventive option for individuals to avoid developing CRC. Berries were shown to reduce the oral dysplasia and carcinoma-in-situ by approximately $50 \%$ in animals previously treated with a mixture of the cigarette smoke carcinogens, B[a]P and NNK [156]. Previous work from our laboratory against breast and lung cancer has shown that Anthos possess chemopreventive as well as therapeutic effects due to their roles as anti-inflammatory, antioxidant agents and modulators of CYP1A1 and CYP1B1 [157]. We demonstrated that intervention with the anthocyanidin, delphinidin favorably modulated the underlying mechanisms of potent PAHs [158]. It should also be noted that work with both anthocyanins and Anthos suggest that these compounds do not influence the expression or activity of key enzymes involved in drug metabolism including CYP3A4, CYP2A6, CYP2B6, or CYP2C9 [159]. Furthermore, although work has been conducted to research the impact of anthocyanins on CRC [97, 160], no research had previously been conducted to study the impact of Anthos or ETBF bacteria on alterations in phase I and II enzyme expression.

With this in mind, the aim of this series of studies was to assess how treatment with a native mixture of anthocyanidins derived from bilberry (Anthos) and an exosomally-delivered preparation of Anthos (ExoAnthos) influences proliferation and modulation of expression of key phase I and II enzymes both in vitro and in a bacterially-induced in vivo model of colorectal cancer. Furthermore, the influence of gut bacterial dysbiosis induced by ETBF on phase $\mathrm{l} / \mathrm{Il}$ enzyme expression was also investigated. 


\section{Materials and Methods}

\section{Chemicals}

$\mathrm{B}[\mathrm{a}] \mathrm{P}$ was handled carefully with all safety procedures as it is a highly carcinogenic and hazardous chemical. B[a]P (B-1760, St. Louis, MO) was purchased from Sigma-Aldrich.

\section{Isolation of bilberry-derived Anthos}

The native bilberry Anthos mixture that was generously provided by $3 \mathrm{P}$ Biotechnologies (Louisville, KY) was further enriched using C18 Sep-Pak cartridges (Waters, Milford, MA) and eluted in acidified $(0.1 \% \mathrm{HCl})$ ethanol. The enriched extract was then dried using a Savant SC210A Speed-Vac (ThermoFisher Scientific, Waltham, MA) and stored at $-20^{\circ} \mathrm{C}$. Purity was verified using HPLC-PDA-UV. Briefly, $15 \mu \mathrm{l}$ samples were analyzed using a Shimadzu Premier C18 reverse-phase column (250x4.6 mm i.d., $5 \mu \mathrm{m})$. Mobile phase A was composed of water: formic acid: acetonitrile (87:10:3) and mobile phase B was composed of water: formic acid: acetonitrile (40:10:50). The flow rate was $0.6 \mathrm{ml} / \mathrm{min}$ and the gradient condition was $0-5 \min 5 \% \mathrm{~B} ; 5-15 \min 15 \% \mathrm{~B} ; 15-20$ $\min 25 \%$ B; $20-30$ min $35 \%$ B; $30-40$ min $45 \%$ B; $40-45 \min 100 \%$ B; $45-50$ min $5 \%$ B. Detection of Anthos was at $520 \mathrm{~nm}$ by PDA-UV and total Anthos concentration was calculated using a standard curve. The reference compounds were purchased from Cayman Chemical Company (Ann Arbor, MI). The HPLC analysis of the Anthos was conducted by Mr. Jeyaprakash Jeyabalan, a Research Scientist in our laboratory. 


\section{Isolation of milk derived exosomes}

Exosomes from cow colostrum were isolated using differential centrifugation as described by Munagala et al., [115], and were generously provided by 3P Biotechnologies (Louisville, KY).

\section{Protein determination}

Protein estimation for exosomal preparations was assessed using a bicinchoninic acid (BCA) assay (Thermo Scientific, Rockford, IL). In order to determine protein concentration, diluted exosomal preparations were compared, in triplicate, to a serially diluted bovine serum albumin (BSA) standard curve.

\section{ExoAnthos drug loading}

Anthos was loaded onto exosomes by mixing Anthos (dissolved in ethanol and water) with the exosomes suspension in a 1:5 (Anthos:Exosomal protein, w/w) ratio at room temperature $\left(22^{\circ} \mathrm{C}\right)$. Unbound Anthos and any co-agulated exosomes were removed by low speed centrifugation $(10,000 \times g$ for $10 \mathrm{~min})$. The

exosomes that were loaded with Anthos were then collected by ultracentrifugation (135,000 $\mathrm{xg}$ for $1.5 \mathrm{~h}$ ). The pellet was then suspended in PBS and passed through a $0.22 \mu$ filter and stored at $-80 \stackrel{\circ}{\circ}$. The percent loading was determined using solvent extraction.

\section{Analysis of drug loading}

In order to determine the load of Anthos in the ExoAnthos formulation, the protein and Anthos concentrations were measured. Briefly, a $50 \mu \mathrm{l}$ aliquot of ExoAnthos 
formulation was mixed with $950 \mu \mathrm{l}$ of acidified ethanol $(0.1 \% \mathrm{HCl})$. The precipitated proteins were separated by centrifugation $(10,000 \times g$ for 10 minutes). The drug contained in the supernatant was then analyzed using a SpectraMax M2 spectrometer (Molecular Devices, Sunnyvale, CA). Anthos were detected at $520 \mathrm{~nm}$ and total Anthos concentration was calculated using a standard curve. Reference anthocyanidins were acquired from Chromadex (Irvine, CA). Anthos concentrations were confirmed via HPLC-PDA. The pelleted exosomal proteins were determined by the BCA method described above. The percent drug load when then calculated by dividing the amount of Anthos by exosomal proteins $x 100$ [115]. Equal loading of individual Anthos was confirmed using HPLC-DAD.

\section{Characterization of Exosomes and ExoAnthos}

The size of colostrum-derived exosomes and ExoAnthos, which were diluted in PBS to $1 \mathrm{mg} / \mathrm{ml}$, were determined using a Zetasizer (Malvern Instruments Ltd, Malvern, Worcestershire, UK). Colostrum exosome and ExoAnthos morphology were determined by atomic force microscopy (AFM). Briefly, exosomes and ExoAnthos were diluted with deionized water to $10 \mu \mathrm{g} / \mathrm{ml}$. A $2 \mu \mathrm{l}$ aliquot of each sample was then placed on a silica wafer and air dried for $30 \mathrm{~min}$. Images were captured using an Asylum MF-3D AFM (Oxford Instruments, Goleta, CA) in tapping mode using aluminum-coated silicon probes. The amplitude, topographic height and phase retraces were imaged with a fixed force $(<1 \mathrm{nN})$ at a scanning rate of $1 \mathrm{~Hz}$. Images were processed using IGOR software. The AFM 
analysis was performed by Mr. Jeyaprakash Jeyabalan, a Research Scientist in our laboratory.

\section{Cells, culture conditions and treatments}

The APC wild-type HCT 116 (ATCC® CCL-247 ${ }^{\mathrm{TM}}$ ) colon cancer cell line and CCD-18Co (ATCC ${ }^{8}$ CRL-1459 ${ }^{\mathrm{TM}}$ ) normal colon cells were acquired from American Type Culture Collection (Manassas, VA). The APC mutant colon cancer cell line HT-29 (ATCC® HTB-38D ${ }^{\mathrm{TM}}$ ) was a kind gift from Dr. Nobuyuki Matoba (University of Louisville). HCT-116 and HT-29 cells were maintained in McCoy's 5A medium (Gibco, Grand Island, NY) supplemented with 10\% FBS, $100 \mathrm{U} / \mathrm{ml}$ penicillin and $100 \mu \mathrm{g} / \mathrm{ml}$ streptomycin in a humidified atmosphere containing $5 \% \mathrm{CO}_{2}$ at $37^{\circ} \mathrm{C}$. Cells were pre-treated with Anthos $(25,50,100,200$ $\mu \mathrm{M})$ for $24 \mathrm{~h}$ and co-treated with Anthos $(25,50,100,200 \mu \mathrm{M})$ and $\mathrm{B}[\mathrm{a}] \mathrm{P}(20 \mu \mathrm{M})$ for $24 \mathrm{~h}$.

\section{Measurement of cell viability}

The cytotoxicity of bilberry Anthos in colon cancer cell lines was assessed by enzymatic reduction of the tetrazolium dye MTT. Briefly, $3.0 \times 10^{3}$ cells/well were grown in a 96-well tissue culture plates and were then exposed to varying concentrations of bilberry Anthos or vehicle control 24 hours after seeding. After $72 \mathrm{~h}$ treatment, cells were incubated with $5 \mathrm{mg} / \mathrm{ml}$ MTT reagent for $2 \mathrm{~h}$. Resulting formazan crystals were subsequently solubilized in DMSO and spectrophotometrically measured at $570 \mathrm{~nm}$ (Bio-rad, Philadelphia, PA). $\mathrm{IC}_{50}$ 
values were then determined using Calcysyn software version 2.1 (Biosoft, Cambridge, England).

\section{Western-blot analysis}

For western-blot analysis, $40 \mu \mathrm{g}$ of protein from in vitro and in vivo tissue lysates was resolved using SDS-polyacrylamide gel electrophoresis and electrotransferred to polyvinylidene difluoride membranes by semi-dry transfer (Biorad Trans-blot SD, Hercules, CA). Blots were blocked with 4\% dry powder milk or BSA for $1 \mathrm{~h}$ and then incubated with primary antibodies $\beta$-actin, UGT1A6, SULT1, GSTM1, GSTM2, CYP1A1, CYP1B1, PXR, Nrf2, AhR, AhRR, and ARNT1 which were all acquired from Santa Cruz Biotech (Santa Cruz, CA) at $4^{\circ} \mathrm{C}$ overnight and secondary antibodies (Santa Cruz Biotech, Santa Cruz, CA). conjugated to peroxidase for $1 \mathrm{~h}$ at room temperature. Blots were then developed with an ECL detection system. Densitometric analysis was then performed using ImageJ 1.x software [134].

\section{Animal model for CRC}

$\mathrm{Apc}^{\mathrm{Min} /+}$ mice are a well established and accepted model for studying FAP and CRC. The mice characteristically exhibit a germline nonsense mutation at codon 850 of the APC gene that causes the spontaneous development of polyps, which predominantly occur in the small intestine by the age of 10-12 weeks.

Enterotoxigenic Bacteriodes fragilis (ETBF) is a highly relevant model for development of CRC due to its contribution to both familial and sporadic forms of 
cancer $[28,135]$. ETBF exists asymptomatically in $12.4 \%$ of individuals overall and in $27 \%$ of individuals with diarrhea symptoms [135]. Furthermore, presence of ETBF in the gut is a well known as a global cause of diarrheal disease that is accompanied by colitis in both humans and animals. The pathogenicity associated with ETBF is due to the secretion of a $20 \mathrm{kDa}$ zinc-dependent metalloprotease toxin, B. fragilis toxin (BFT), which binds to colonic epithelial cells and leads to the cleavage of the tumor suppressor protein, E-cadherin, and the secretion of interleukin-8 [136]. Overall, this process leads to the stimulation of proliferation and migration of human colon cancer cells [137]. It should also be noted that BFT has also been shown to induce pro-inflammatory cytokine secretion by further activating the NFKB pathway [137].

\section{In vivo CRC studies}

Animal experiments were performed in agreement with an approved protocol by the Institutional Animal Care and Use Committee at the University of Louisville. Breeding colonies were established in collaboration with Dr. Nejat K. Egilmez's lab [138] at the University of Louisville using C57BL/6J Min/+ $\left(\mathrm{Apc}^{\mathrm{Min} /+}\right)$ mice that were originally attained from Jackson Laboratories (Bar Harbour, ME). Mice were genotyped for the APC mutation using PCR according to the protocol established by Jackson Laboratories. Mice were fed a standard chow diet and received water ad libitum and were maintained on a standard light/dark cycle for the duration of the study. At 5-6 weeks of age, animals were administered antibiotic. Four days later the animals were administered ETBF to promote tumorigenesis and one 
week following ETBF inoculation, animals began their respective treatment regimen. Male and female $\mathrm{Apc}^{\mathrm{Min} / \mathrm{+}}$ mice were orally administered (by gavage) an average of $8 \mathrm{mg} / \mathrm{kg} /$ day Anthos, $8 \mathrm{mg} / \mathrm{kg} /$ day ExoAnthos or vehicle control for 4 weeks. Animals were culled in the fed state at 12 weeks, colon tumors were counted and tissues were harvested.

\section{Data analysis}

Statistical analysis was performed using Graph Pad Prism statistical software version 4.03 (La Jolla, CA) and RStudio software version 1.0.153 (Boston, MA) Lattice package [139, 140]. Test for normality, F-test for equal variance, and appropriate t-test were used for both animal studies and for analysis of western blot results. One way ANOVA was used for assessing the significance of mean differences across the various treatments for western data. $I_{50}$ values were determined using CalcuSyn software version 2.1 (Biosoft, Cambridge, England). Heat maps were constructed using RStudio software version 1.0.153 (Boston, MA) gplot package $[140,141]$.

\section{Results}

\section{Anti-proliferative effects of Anthos and ExoAnthos on colon cancer cells but not normal colon cells}

Previous work from our laboratory has shown that exosomal formulation yielded enhanced therapeutic potency and efficacy for drugs such as paclitaxel, celastrol, curcumin and Anthos against lung cancer due to increased stability and 
bioavailability of these compounds $[115-117,142]$. Prior to carrying out our in vivo work, we first determined the impact of ExoAnthos, exosomal vehicle (Figure 14) and Anthos treatment on proliferation of HCT-116 and HT-29 colon cancer cell lines and CCD-18Co normal colon cells (Figure 15). Results from these studies showed a clear increase in the anti-proliferative properties of Anthos against colon cancer cells, with 4-16-fold decreases in the $\mathrm{IC}_{50}$ values of ExoAnthos as compared to the free Anthos (Figure 15). One can posit that the improved anti-proliferative effects of the ExoAnthos formulation over Anthos alone is most likely due to the increased cell uptake and stability in media of the ExoAnthos over Anthos. Part of the higher efficacy of ExoAnthos may be attributed to the intrinsic effect of the exosomes alone (Munagala et al. 2016, 2017).

In order to determine whether Anthos and ExoAnthos were selective toward colon cancer over normal colon cells in vitro we determined the selectivity index (SI) values for both HCT 116 and HT-29 colon cancer cells compared to normal colon CCD-18Co cells. The results (Table 2) showed not only that both Anthos and ExoAnthos were selective for colon cancer over normal colon cells, but that ExoAnthos enhanced this selectivity, with the greatest increase yielded in HT-29 cells which went from an SI value of 9 for Anthos to 51 for ExoAnthos. Overall, these results confirm that Anthos and ExoAnthos do not show in vitro toxicity for the normal CCD-18Co colon cells and that the cytotoxicity is specific for colon cancer cells. 


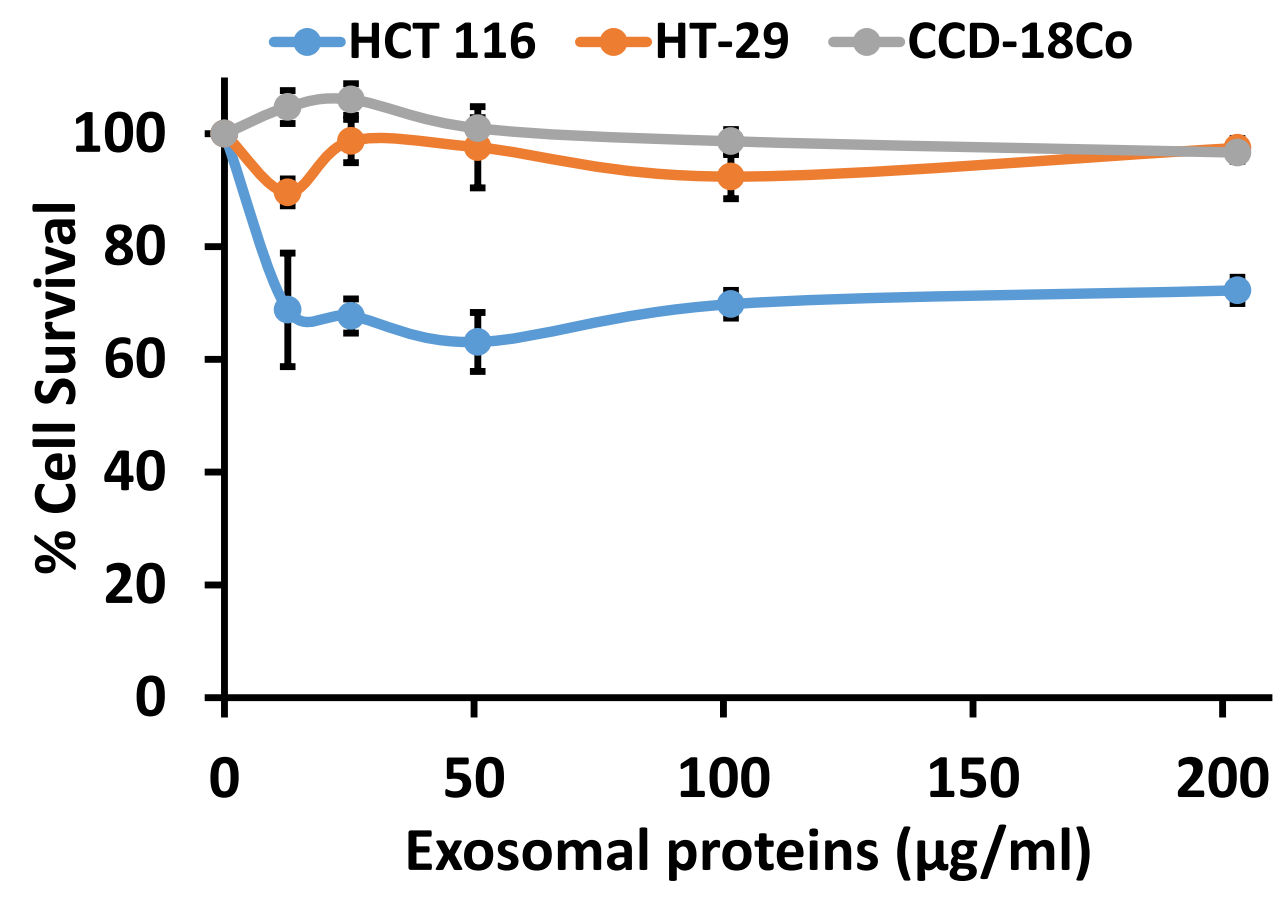

Figure 14. Antiproliferative activity of Exosomes against colon normal cells and cancer cell lines: Colon normal cells CCD-18Co and colon cancer cell lines HCT-116 and HT-29 were treated with various concentrations of milk derived exosomes for $72 \mathrm{~h}$ and the effect on cell growth inhibition was assessed using an MTT assay. Data represent average \pm SEM $(n=3)$. 

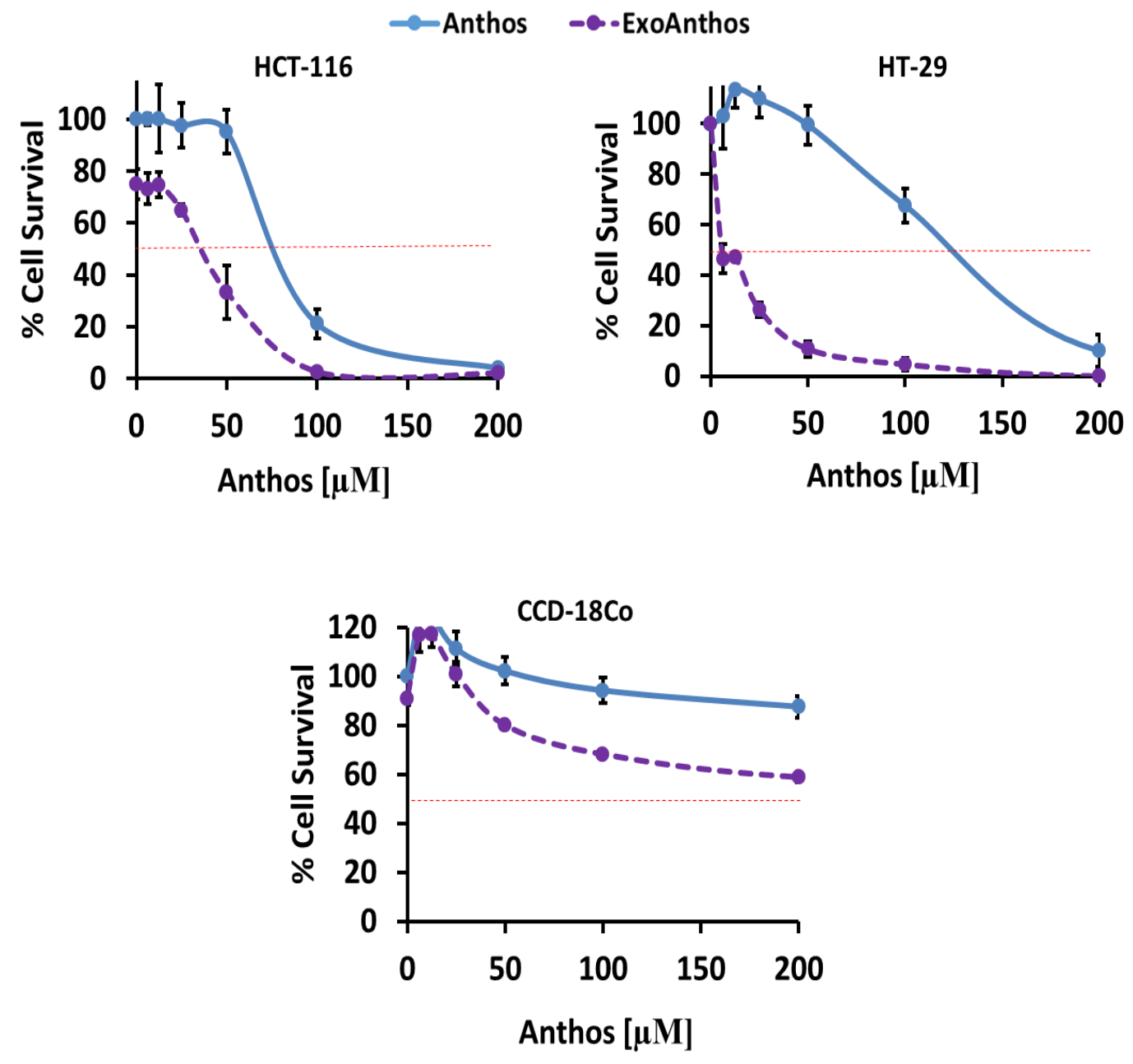

Figure 15. Antiproliferative activity of Anthos and ExoAnthos against colon normal cells and cancer cells in vitro: Colon normal cells, CCD-18Co and colon cancer cells, HCT-116 and HT-29 were treated with various concentrations of bilberry-derived Anthos or ExoAnthos for $72 \mathrm{~h}$ and the effect on cell growth inhibition was assessed using an MTT assay. Data represent average \pm SEM $(n=4)$. 
Table 2. Tabulated selectivity values for colon cancer and normal cells treated with Anthos and ExoAnthos

\begin{tabular}{|c|c|c|c|c|c|}
\hline Cell line & $\begin{array}{c}\mathrm{IC}_{50} \text { Anthos } \\
{[\mu \mathrm{M}]}\end{array}$ & SI Anthos & $\begin{array}{c}\mathrm{IC}_{50} \text { ExoAnthos } \\
{[\mu \mathrm{M}]}\end{array}$ & SI ExoAnthos & $\begin{array}{c}\text { Fold } \\
\text { difference in } \\
\mathrm{IC}_{50} \text { values }\end{array}$ \\
\hline CCD-18Co & 1050 & - & 407 & - & - \\
\hline HCT116 & 75 & 14 & 20 & 20 & 4 \\
\hline HT-29 & 124 & 9 & 8 & 51 & 16 \\
\hline
\end{tabular}




\section{Impact of Anthos treatment on tumor number in vivo}

As presented in chapter 2, Anthos led to a significant reduction in colon tumors in

an ETBF Apc ${ }^{\mathrm{min} /+}$ mouse model when given at an average dose of $16 \mathrm{mg} / \mathrm{kg}$. Given these promising results, we next sought to test our ExoAnthos formulation, which makes use of an exosomal nano delivery method to see if greater therapeutic effect could be achieved at lower doses due to enhanced stability and bioavailability of the Anthos. We have previously found that Exosomal delivery enabled us to achieve superior results for several drugs such as curcumin, celastrol and paclitaxel when given alone in vivo for breast and lung cancer [115-117, 144]. Results from the comparison of Anthos and ExoAnthos treatment showed that ExoAnthos lead to a similar reduction in colon tumor burden as Anthos alone, when given at the same dose $(P=0.30)$. When compared to control, a significant reduction in colon tumors was noted in the ExoAnthos treated animals vs. exosome vehicle control $(P=0.019)$ and Anthos treated animals vs. vehicle control $(P=0.0025)$ when given at $8 \mathrm{mg} / \mathrm{kg}$ (Figure 16). It should be noted that no significant difference was found between the tumor numbers in control vs. exosome alone treated animals $(P=0.728)$.

\section{Impact of ETBF bacteria on phase $\mathrm{I} / \mathrm{ll}$ enzyme expression in colon/liver}

Prior to this study, no work had been completed assessing the impact of ETBF bacteria on the expression of phase I or II enzymes. However, there has been growing interest in the scientific community regarding the impact of the gut 


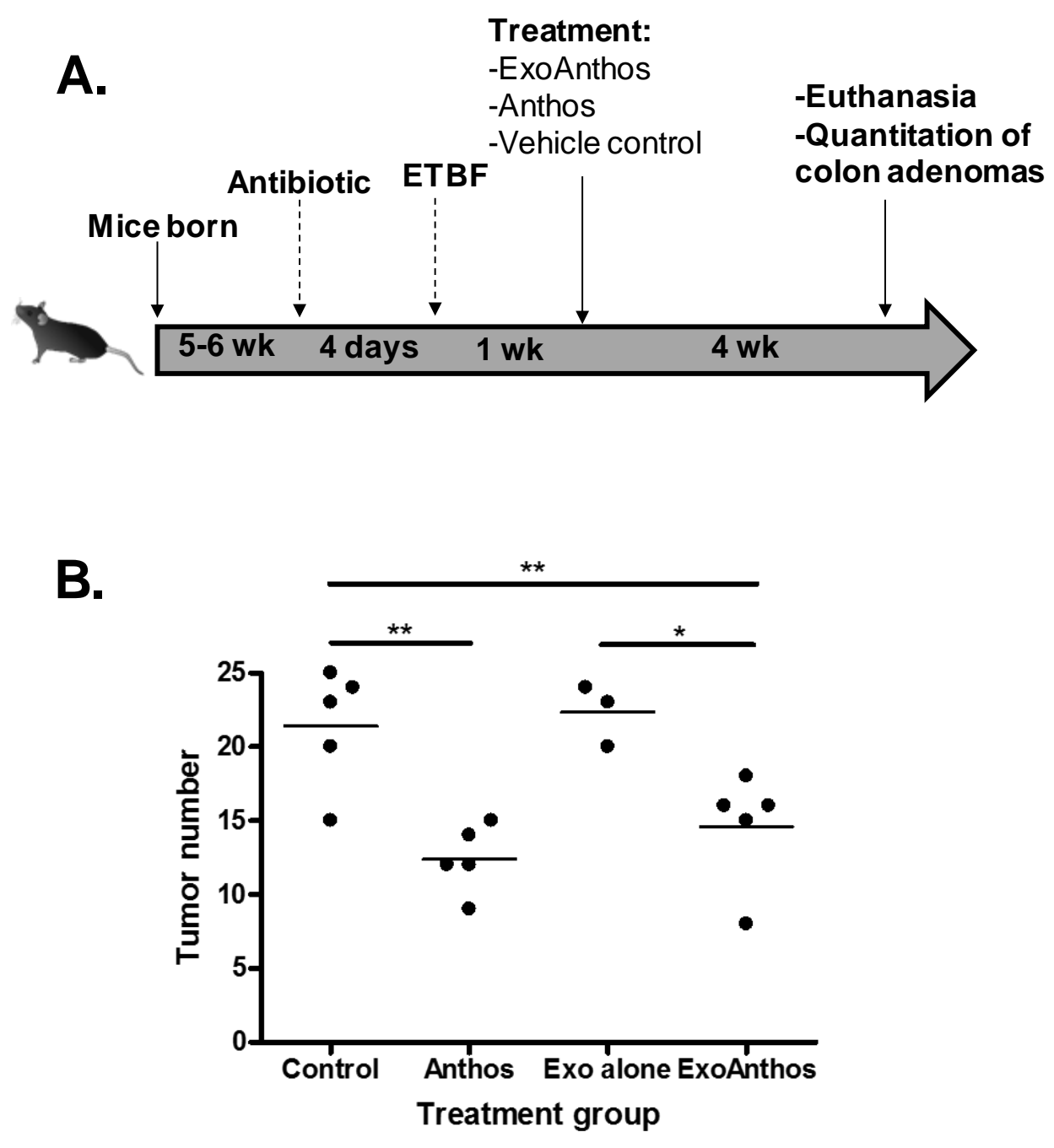

Figure 16. Anti-tumor activities of Anthos and ExoAnthos against colon tumors. (A) Study overview, $\mathrm{Apc}^{\mathrm{Min} /+}$ mice inoculated with ETBF were treated via oral gavage with Anthos or ExoAnthos three times a week (20 mg/kg bw) or vehicle controls. (B) Data represent the distribution of animal colon tumor counts, with the average noted. Anthos versus control $(P=0.0025)$, control versus exosomes alone-treated animals $(P=0.728)$, ExoAnthos treated animals versus exosomes vehicle control $(P=0.019)$, Anthos versus ExoAnthos $(P=0.30) .{ }^{* * *}$ signifies $P<0.001,{ }^{* *}$ signifies $P<0.01$, and ${ }^{*}$ signifies $P<0.05$. 
microbiome on the metabolism of drugs and environmental chemicals such as polybrominated diphenyl ethers (PBDEs) [161]. Results from our investigation of the influence of ETBF bacteria on the expression of phase I and II enzymes in $\mathrm{Apc}^{\mathrm{Min} /+}$ mice treated with the bacteria showed significant increases in the expression of the phase I enzymes CYP1A1 and CYP1B1. Additionally, a significant decrease in the expression of the phase II enzyme GSTM1 in normal colon tissue was also noted. In order to assess how ETBF bacteria influences the expression of these phase I and II enzymes we assessed the expression of AhR, AhRR, ARNT1, Nrf2, VDR and PXR in $\mathrm{Apc}^{\mathrm{Min} /+}$ mice with and without ETBF treatment. Results from this survey (Figure 17) showed that mice treated with bacteria had highly significant decreases in the expression of the AhR repressor, AhRR. No significant changes in the expression of PXR, Nrf2, and VDR expression were noted in colon tissue samples taken from the mice that received bacteria. Liver samples taken from bacteria-treated mice also featured decreased expression of the phase II enzymes UGT1A6 and GSTM1 and increased expression of AhR when compared to $\mathrm{Apc}^{\mathrm{Min} /+}$ mice that did not receive bacteria.

\section{Impact of Anthos treatment on phase $1 / 1$ ll enzyme expression in vivo: colon/liver}

In chapter 2, we showed that Anthos treatment led to decreases in the phosphorylation status of both Src (Y418) and EGFR (Y845) in both tumor and 


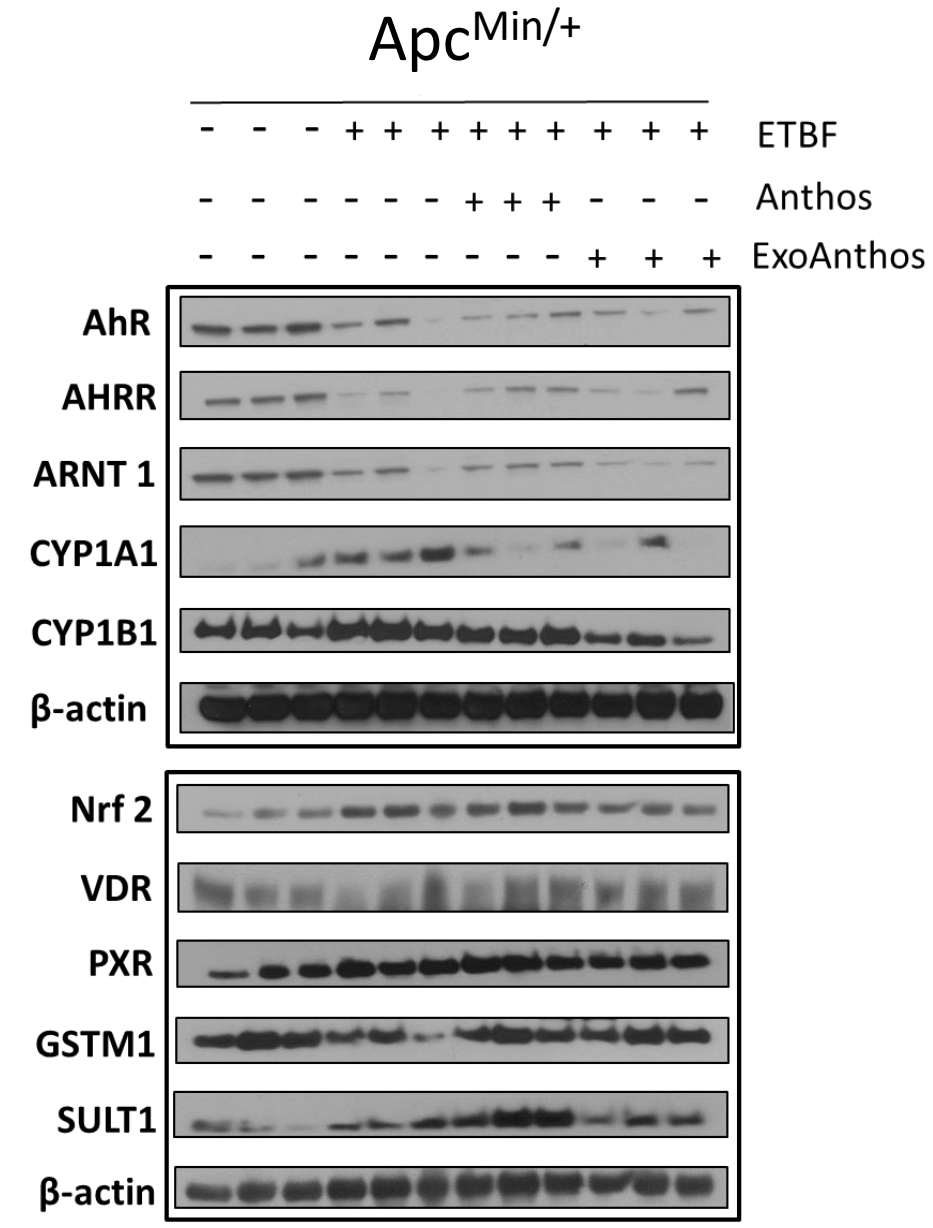

Figure 17. In vivo changes in phase I and II enzyme expression and related xenobiotic sensing nuclear receptors in normal colon tissue following treatment with ETBF bacteria, bilberry-derived Anthos or ExoAnthos: changes in the expression of key phase I and II enzymes along with key nuclear receptors including CYP1A1, CYP1B1, GSTM1, SULT1, AhR, AhRR, ARNT1, Nrf2, PXR and VDR in normal colon tissue taken from ETBF-inoculated $\mathrm{Apc}^{\mathrm{Min} /+}$ mice after treatment with bilberry-derived Anthos, ExoAnthos or vehicle control as assessed using western blot analysis compared to with $\mathrm{Apc}^{\mathrm{Min} /+}$ mice that received no bacteria and $\beta$-actin loading control. 

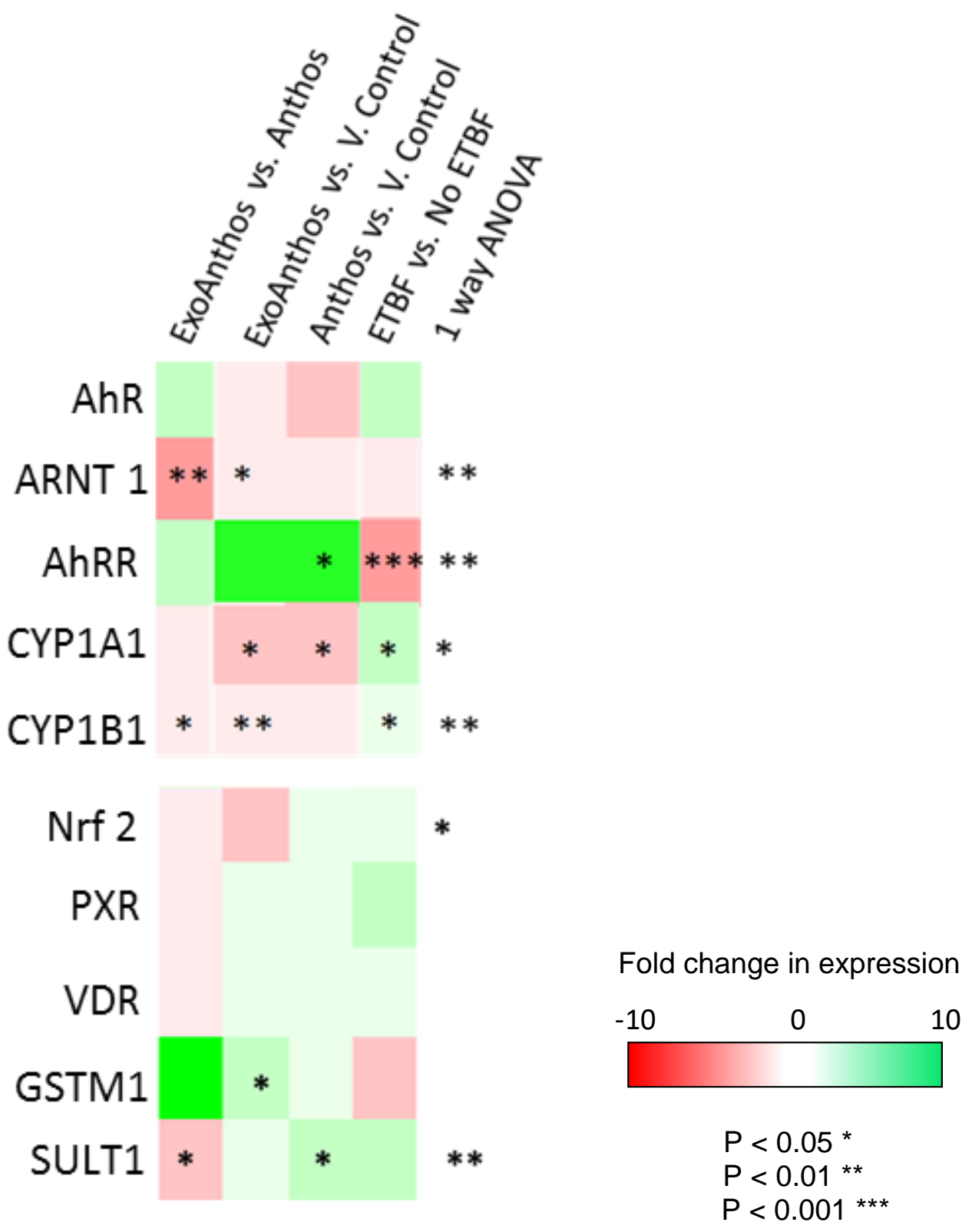

Figure 18. Representative heatmaps for In vivo changes in phase I and II enzyme expression and related xenobiotic sensing nuclear receptors. Quantitative data summarized in Table 3. 
Table 3. Raw densitometry values calculated for western blot analysis of phase I \& II enzyme expression and related xenobiotic sensing nuclear receptors in normal colon tissue

\begin{tabular}{|c|c|c|c|c|c|c|c|}
\hline \multirow{2}{*}{ Target } & \multicolumn{7}{|c|}{ Normal Colon } \\
\cline { 2 - 7 } & $\begin{array}{c}\text { ApcMin/+, } \\
\text { No ETBF }\end{array}$ & Control & $\begin{array}{c}\text { Fold } \\
\text { change } \\
\text { (ETBF } \\
\text { vs. No } \\
\text { ETBF) }\end{array}$ & Anthos & $\begin{array}{c}\text { Fold } \\
\text { change } \\
\text { (Control vs. } \\
\text { Anthos) }\end{array}$ & ExoAnthos & $\begin{array}{c}\text { Fold change } \\
\text { (Control vs. } \\
\text { ExoAnthos) }\end{array}$ \\
\hline AhR & 58410 & 29526 & -2 & 17585 & -2 & 14032 & -2 \\
\hline AhRR & 53603 & 13357 & -4 & 27057 & 2 & 21555 & 2 \\
\hline ARNT 1 & 58885 & 40294 & -1 & 34663 & -1 & 15608 & -3 \\
\hline CYP1A1 & 2726 & 9826 & 4 & 8187 & -1 & 4482 & -2 \\
\hline CYP1B1 & 3384 & 9273 & 3 & 9723 & 1 & 6887 & -1 \\
\hline Nrf2 & 37037 & 59867 & 2 & 58756 & -1 & 44028 & -1 \\
\hline VDR & 27347 & 32973 & 1 & 50719 & 2 & 45036 & 1 \\
\hline PXR & 42009 & 69029 & 2 & 72372 & 1 & 60007 & -1 \\
\hline GSTM1 & 6380 & 5734 & -1 & 11143 & 2 & 7378 & 1 \\
\hline SULT1 & 7888 & 7061 & -1 & 11076 & 2 & 10823 & 2 \\
\hline
\end{tabular}


adjacent normal tissue, and EGFR (Y1068 and Y1173) in colon tumor tissue. However, no work had been conducted to assess whether Anthos modulated phase I and II enzyme expression in colon or liver tissue in animals treated with ETBF bacteria. Results from our survey of the impact of Anthos and ExoAnthos on key phase I and II enzymes involved in the metabolism of the environmental carcinogen $\mathrm{B}[\mathrm{a}] \mathrm{P}$ as well as other carcinogens, demonstrated significant modulation of the phase I enzymes CYP1A1 and CYP1B1 and phase II enzymes GSTM1 and SULT1 by Anthos and ExoAnthos (Figures 17-18) in normal colon tissue. Our survey of nuclear transcription factors and associated proteins including AhR, AhRR, ARNT1, Nrf2, VDR and PXR found that Anthos led to increases in the expression of the AhR repressor AhRR when compared to ETBF alone treated animals. Interestingly, ExoAnthos treatment was not shown to increase expression of AhRR but rather it led to decreased expression of the aryl hydrocarbon receptor nuclear translocator, ARNT1. No significant changes were noted in the expression of PXR, Nrf2, or VDR in colon samples from Anthos or ExoAnthos treated animals. Results from the enzyme expression analysis of liver tissues taken from the same animals noted similar decreases in the expression of CYP1A1 in Anthos treated mice and increases in the phase II enzymes GSTM1 and UGT1A6 (Figures 19-20). No significant changes in expression of mEH or SULT1 were noted in liver tissue. A significant decrease in AhR was noted in ExoAnthos treated mice and a significant increase in AhRR expression was noted in Anthos treated as compared to ETBF Apc ${ }^{\mathrm{Min} /+}$ vehicle control mice. 


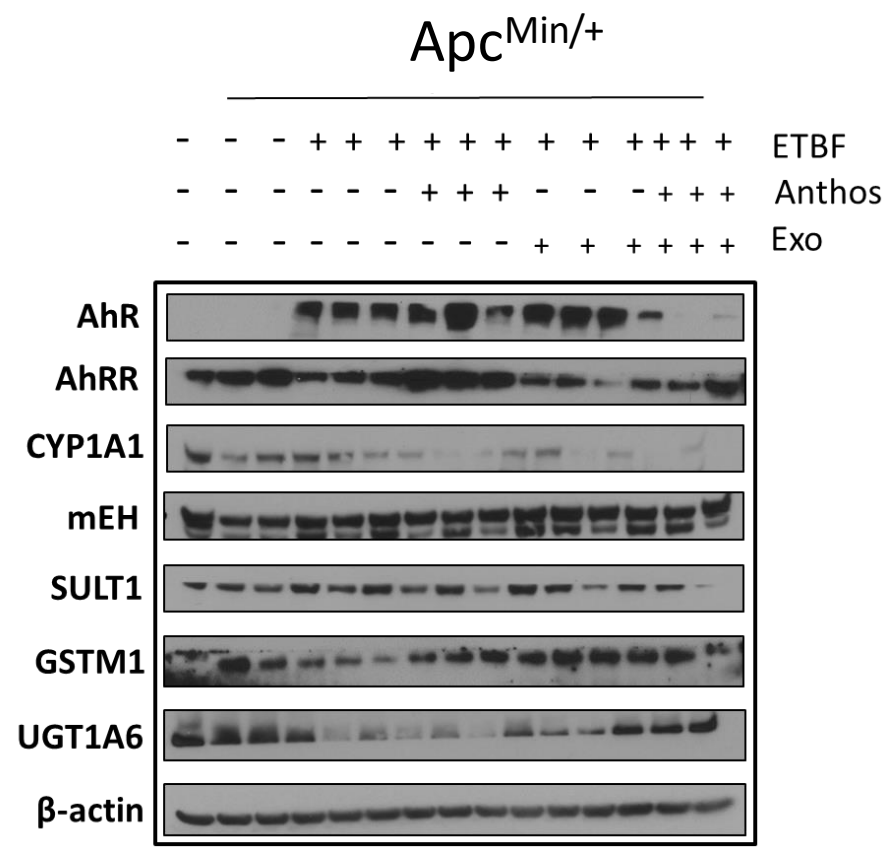

Figure 19. In vivo changes in phase I and II enzyme expression and related xenobiotic sensing nuclear receptors in liver tissue following treatment with ETBF bacteria, bilberry-derived Anthos or ExoAnthos: changes in the expression of key phase I and II enzymes along with key nuclear receptors including CYP1A1, mEH, GSTM1, SULT1, AhR, AhRR, in liver tissue taken from ETBF-inoculated $\mathrm{Apc}^{\mathrm{Min} /+}$ mice after treatment with bilberry-derived Anthos, ExoAnthos or vehicle control. Assessed using western blot analysis compared to with $\mathrm{Apc}^{\mathrm{Min} /+}$ mice that received no bacteria and $\beta$-actin loading control. 


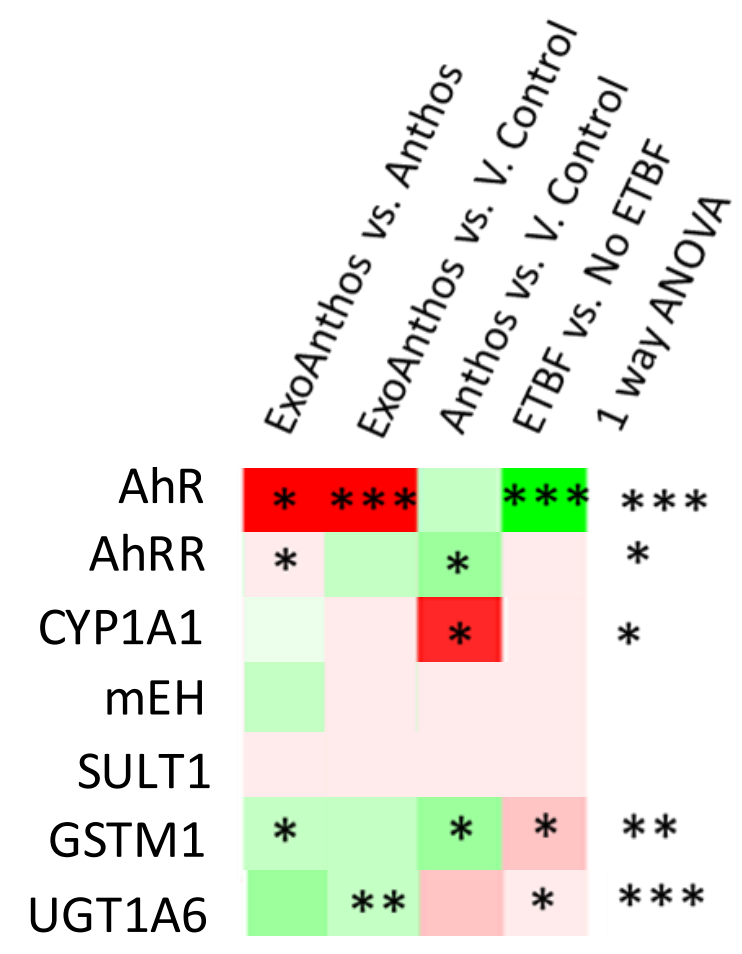

Fold change in expression

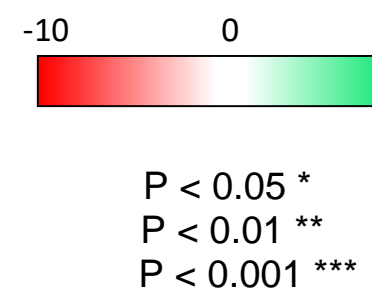

Figure 20. Representative heatmaps for In vivo changes in phase I \& II enzyme expression and related xenobiotic sensing nuclear receptors in liver tissue: heatmaps and significance levels produced from western blot data and related statistical analysis. Quantitative data summarized in Table 4. 
Table 4. Raw densitometry values calculated for western blot analysis of phase I \& II enzyme expression and related xenobiotic sensing nuclear receptors in liver tissue

\begin{tabular}{|c|c|c|c|c|c|c|c|c|c|}
\hline Target & $\begin{array}{c}\text { ApcMin/+ } \\
\text { No } \\
\text { ETBF }\end{array}$ & Control & $\begin{array}{c}\text { Fold } \\
\text { change } \\
\text { (ETBF } \\
\text { vs. No } \\
\text { ETBF) }\end{array}$ & $\begin{array}{c}\text { Exo } \\
\text { Alone }\end{array}$ & $\begin{array}{c}\text { Fold } \\
\text { change } \\
\text { (Control } \\
\text { vs. Exo) }\end{array}$ & Anthos & $\begin{array}{c}\text { Fold } \\
\text { change } \\
\text { (Control } \\
\text { vs. } \\
\text { Anthos) }\end{array}$ & $\begin{array}{c}\text { ExoAnth } \\
\text { os }\end{array}$ & $\begin{array}{c}\text { Fold } \\
\text { change } \\
\text { (Contro } \\
\text { I vs. } \\
\text { ExoAnt } \\
\text { hos) }\end{array}$ \\
\hline AhR & 943 & 34419 & 37 & 44576 & 1 & 38634 & 1 & 6464 & -7 \\
\hline AhRR & 51013 & 38000 & -1 & 26864 & -1 & 71099 & 2 & 42221 & 2 \\
\hline CYP1A1 & 40740 & 30904 & -1 & 20889 & -1 & 9504 & -3 & 9614 & -2 \\
\hline mEH & 28247 & 30977 & 1 & 37739 & 1 & 30286 & -1 & 36194 & -1 \\
\hline SULT1 & 37873 & 55699 & 1 & 46368 & -1 & 46320 & -1 & 32923 & -1 \\
\hline GSTM1 & 34244 & 11042 & -3 & 29548 & 3 & 19038 & 2 & 26604 & -1 \\
\hline UGT1A6 & 60110 & 26603 & -2 & 21889 & -1 & 13159 & -2 & 42303 & 2 \\
\hline
\end{tabular}




\section{Expression in tumor tissue}

Alterations of phase I and II enzymes have been attributed to being one of the causes for the development of chemo-resistance in cancer [162, 163]. Since modulation of phase I and II enzymes were demonstrated in normal colon and liver tissues, we sought to determine whether additional chemo-resistance could arise for individuals undergoing chemotherapy if they were taking Anthos. In order to do this, we assessed the expression of the phase II enzymes: GSTM1, SULT1, and UGT1A6 in the tumor tissue taken from Anthos treated vs. ETBF control mice. Results from this analysis (Figure 21) showed that Anthos treatment led to no significant changes in the expression of the phase II enzymes: UGT1A6, GSTM1, or SULT1 in the tumor tissue.

\section{Impact of Anthos treatment on alterations induced by $B[a] P$ treatment in} vitro

Alterations in the expression of phase I enzymes had been previously reported by our laboratory in an $\mathrm{ACl}$ rat model for breast cancer [157]. Given this background, we next sought to determine if Anthos and ExoAnthos treatment would alter this shift in phase I/II enzyme expression induced by $B[a] P$ treatment, in vitro. Results (Figure 22) showed that cells treated overnight with $\mathrm{B}[\mathrm{a}] \mathrm{P}(20$ $\mu \mathrm{M})$ featured increased expression of CYP1A1 with decreased expression of GSTM1. Furthermore, treatment with Anthos led to significant decreases in the expression of CYP1A1 while significantly increasing the phase II detoxifying enzymes GSTM1 and SULT1. Anthos treatment, at the highest doses, led to approximately 

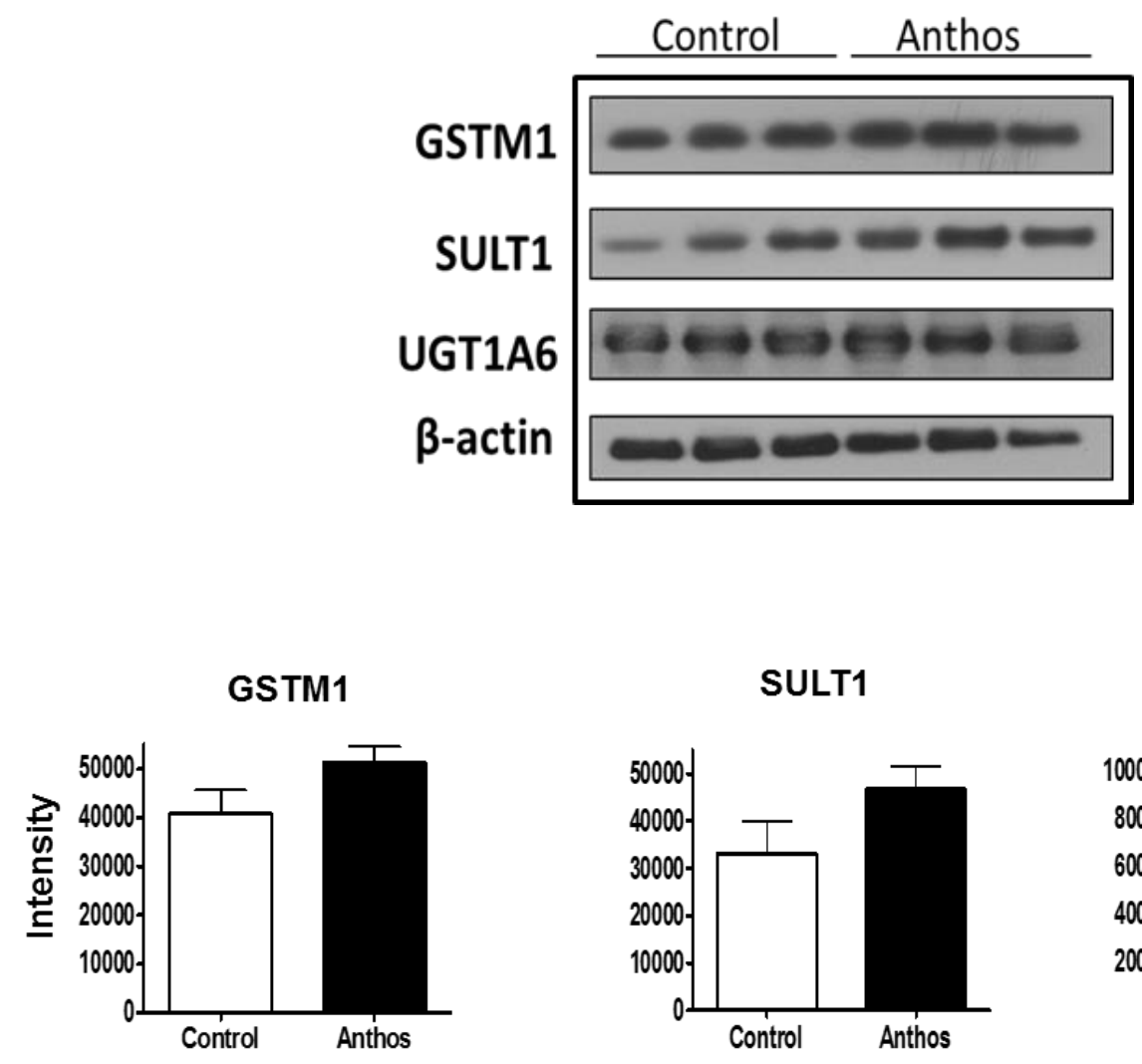

SULT1

UGT1A6
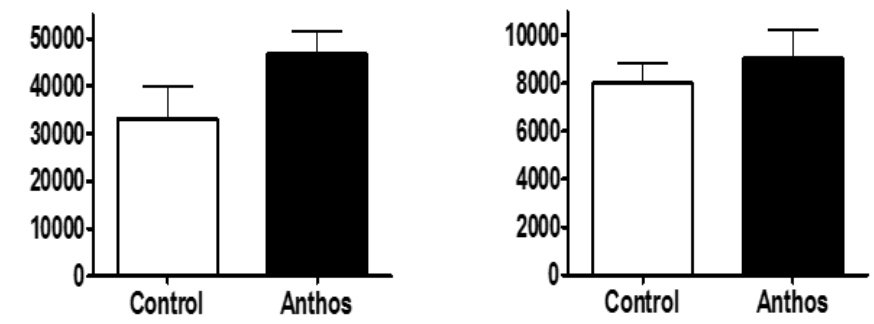

Figure 21. Impact of in vivo Anthos treatment on phase II enzyme expression in colon tumor tissue: changes in the expression of key phase II enzymes including GSTM1 ( $P>0.2)$, SULT1 $(P>0.2)$ and UGT1A6 $(P>0.5)$ taken from ETBF-inoculated $\mathrm{Apc}^{\mathrm{Min} /+}$ mice after treatment with bilberry-derived Anthos or vehicle control as assessed using western blot analysis compared to $\beta$-actin loading control. 
3-fold reductions in the expression of a key transcription factor AhR, which is involved in the expression of CYP1A1, in HCT-116 colon cancer cells. Furthermore, Anthos treatment simultaneously led to a nearly 2 -fold increase in the expression of the AhR repressor, AhRR. Anthos treatment also led to 2- to greater than 10-fold increases in the expression of a key nuclear receptor, PXR, which regulates the detoxification process by upregulating enzymes UDPGT, GSTs and SULTs when it identifies the presence of foreign potentially toxic substrates [164]. It should be noted that the expression was enhanced, although to a lesser extent than Anthos treatment, when in the presence of $B[a] P$ alone. 


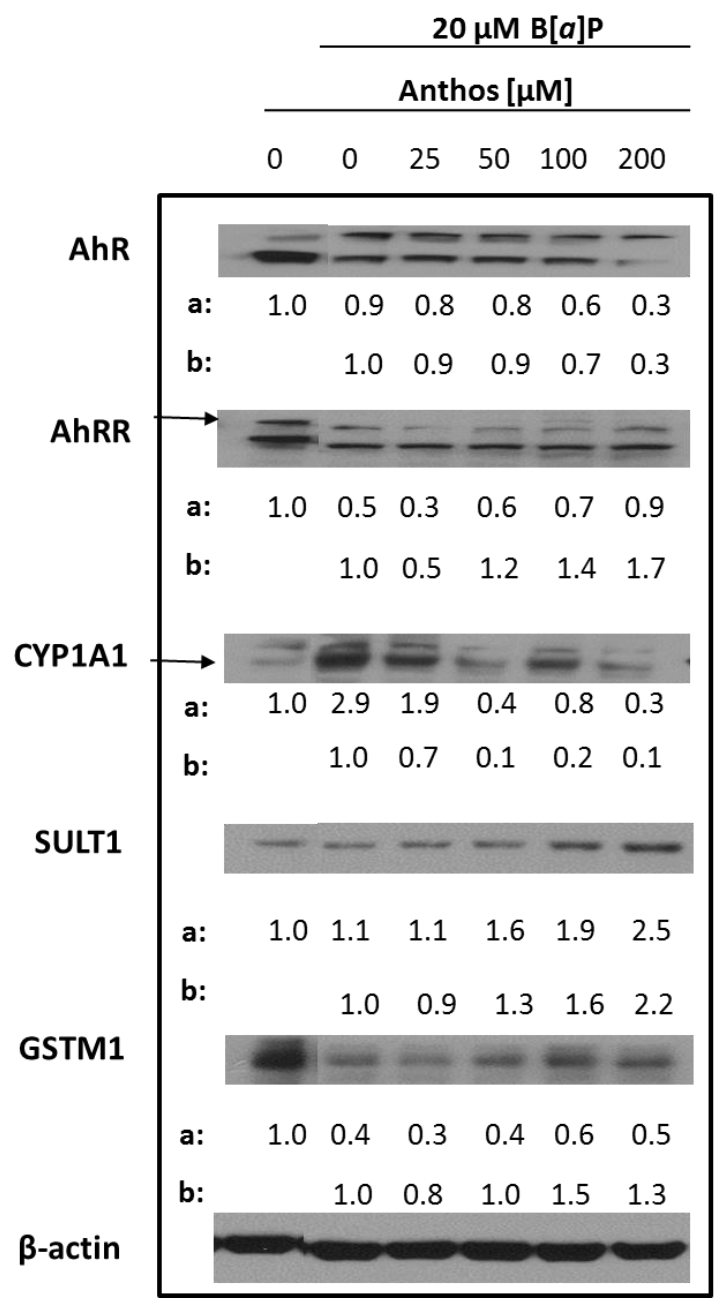

Figure 22. Changes in expression of key phase I and II enzymes and related nuclear receptors following treatment with $B[a] P$ versus $B[a] P$ and Anthos. Colon cancer cell line HCT-116 was pre-treated with various concentrations of Anthos for $24 \mathrm{~h}$ followed by co-treatment with $\mathrm{B}[\mathrm{a}] \mathrm{P}$ and Anthos for $24 \mathrm{~h}$ and the effect on expression of AhR, AhRR, CYP1A1, SULT1, and GSTM1 was assessed using western blot analysis and compared to $\beta$-actin loading control. Densitometry values listed are the ratio of each dose to the vehicle control (a) and $\mathrm{B}[\mathrm{a}] \mathrm{P}$ control (b), corrected for $\beta$-actin loading control. Treatment and controls were run on the same gels. 


\section{Discussion}

Given the recent uptick in cases of CRC diagnosed in younger individuals [12], CRC appears to be making a comeback and thus warrants research into identifying potential chemopreventive methods to combat this disease. Although much research has been conducted on the role of carcinogens such as $\mathrm{B}[a] \mathrm{P}$ on the development of colorectal cancer, little has been achieved in successful prevention of this disease in populations who are exposed to such environmental carcinogens on a daily basis. It should be noted that many studies have been conducted on a variety of plant-derived chemopreventive agents but few agents have successfully been translated, perhaps due to a generalized poor understanding of how they function mechanistically and issues pertaining to bioavailability. For instance, anthocyanins have been attributed with a variety of health-promoting benefits including chemopreventive and therapeutic effects due to their roles as anti-inflammatory and antioxidant agents [94, 131]. However, no studies investigating the impact of Anthos on the balance of phase $\mathrm{I} / \mathrm{II}$ enzymes in the colon had been reported in the literature. Furthermore, the impact of bacteria such as ETBF on this balance in the colon and liver has also yet to be elucidated. Given the lack of understanding/knowledge in these areas, we sought to uncover the impact of ETBF bacteria, Anthos and $B[a] P$ on this crucial enzymatic balance. 


\section{Bacteria and role of ETBF in modulating phase I/II enzymes}

Several recent studies have confirmed the link between dysbiosis of the gut microbiome and colorectal cancer. Several bacteria in particular have been associated with increased risk of developing CRC including: S. gallolyticus, $\mathrm{H}$. pylori, virulent forms of Escherichia coli (E. coli), Fusobacterium nucleatum ( $F$. nucleatum), Salmonella enterica (S. enterica) and enterotoxigenic B. fragilis [155]. Although much research has been conducted on the role of bacteria on inflammation and carcinogenesis [28, 147, 165],not much has been uncovered on the role of ETBF on the expression of phase I and II enzymes. Results from our assessment of the impact that ETBF has on the expression of phase I and II enzymes in $\mathrm{Apc}^{\mathrm{Min} /+}$ mice showed that treatment with the bacteria led to significant increases in the expression of the phase I enzymes CYP1A1 and CYP1B1 in normal colon tissue. Furthermore, highly significant increases in the expression of AhR and significant decreases in the expression of the phase II enzymes GSTM1 and UGT1A6 were noted in liver tissue samples taken from ETBF mice when compared to mice that did not receive the bacteria. The results gathered in this study provide an additional link between how "bad" bacteria such as ETBF can ultimately lead to the development of cancer beyond the initially elucidated inflammatory and gut barrier breakdown pathways [137, 147]. 


\section{Exo Anthos, Anthos modulate expression of phase $\mathrm{I} / \mathrm{Il}$ enzymes in normal}

but not colon tumor tissue

After elucidating how ETBF bacteria increases the expression of phase I enzymes while decreasing the expression of the phase II enzymes in normal colon and liver tissue, we next wanted to determine if Anthos or ExoAnthos treatment could alter this enzyme imbalance. We have previously reported that Anthos treatment led to reductions in the phosphorylation status of Src, EGFR, STAT3, STAT5 and expression of COX-2, Cyclin D1 and Cyclin D2 in this animal model (Chapter 2). Furthermore, previous work from our laboratory has shown that Anthos treatment led to decreased expression and activity of CYP1A1 and CYP1A2 in a breast cancer model [157]. Results from this series of studies showed that Anthos and ExoAnthos treatment significantly decreased the expression of phase I enzymes CYP1A1 and CYP1B1, while increasing the expression of the phase II enzymes GSTM1 and SULT1. Our survey of AhR, ARNT1 and AhRR expression suggest that the modulation of phase I and II enzymes could be attributable to the altered expression of AhRR and ARNT1 induced by Anthos and ExoAnthos treatment. It should be noted that AhRR is a key protein in the AhR signaling cascade that acts as a repressor of AhRdependent gene expression. Structural work shows that AhRR acts by competitively repressing AhR binding to ARNT and target DNA [166] . Furthermore, AhRR levels have been shown to decline in a variety of disease states ranging from rheumatoid arthritis [167] to lung cancer [168]. Interestingly, DNA methylation at AHRR has also been shown to be a marker for smoking and 
was correlated with future smoking morbidity and mortality [169]. As noted above, ARNT1 is also a key component of the AhR signaling cascade and functions by binding to the ligand bound form of $A h R$ and aiding in the movement of the AhR complex to the nucleus. ARNT has also been shown to be upregulated under hypoxic conditions by a HIF-1a dependent mechanism in Hep3B cells [170].

Importantly, no significant changes in the expression of the phase II enzymes UGT1A6, SULT1 or GSTM1 were noted in colon tumor tissue taken from animals treated with Anthos. Therefore, potential negative effects due to increased breakdown of chemotherapeutic drugs that may result from increased phase II enzyme expression, as evolves in many tumors, should not be a cause for concern with Anthos treatment. With this in mind, the selective increase in phase Il enzyme expression in normal tissue over tumor tissue may actually be an advantage in decreasing off target toxicity to healthy tissue for drugs such as

irinotecan [171]. However, additional studies would be needed to confirm this hypothesis.

\section{Influence of $\mathrm{B}[\mathrm{a}] \mathrm{P}$ and Anthos treatment on phase I and II enzyme expression}

Exposure to environmental pollutants is now considered to be one of the reasons behind the increasing rates of individuals with disorders ranging from obesity and type 2 diabetes to cancer $[151,153]$. Up to $90 \%$ of CRC cases are of sporadic origin and it is estimated that diet contributes to $80 \%$ of known cases of CRC. 
The role of chemicals that contaminate food and ultimately contribute to the development of CRC has been of great interest [153]. A class of compounds that is particularly relevant is PAHs. The $\mathrm{PAH}, \mathrm{B}[\mathrm{a}] \mathrm{P}$ is of special interest due to its presence in a variety of common sources of exposure ranging from charcoal cooked food to cigarette smoke and several environmental sources and its epidemiological correlation with increased risk of CRC. Similar to the alterations in phase I and II metabolism found to exist in vivo following ETBF inoculation, we found that cells treated overnight with $\mathrm{B}[\mathrm{a}] \mathrm{P}(20 \mu \mathrm{M})$ featured increased expression of the phase I enzyme CYP1A1 with decreased expression of the phase II enzyme GSTM1 as well as the AhR repressor, AhRR. These results suggest that dysbiosis of the gut microbiome and exposure to the environmental carcinogen $\mathrm{B}[\mathrm{a}] \mathrm{P}$ both lead to dysfunction of the balance between phase I and II enzymes in colon tissue. Furthermore, treatment with Anthos effectively shifted this balance in expression levels of AhRR, AhR, PXR, CYP1A1, and SULT1 to greater favor a state of detoxification in $\mathrm{B}[\mathrm{a}] \mathrm{P}$ treated cells. It should be noted that the beneficial effects attributed to consumption of Anthos in this study may vary from person to person depending upon the presence of genetic polymorphisms of these enzymes. However, since the expression of several enzymes were shown to be modulated by these compounds, individuals may still benefit overall by way of alternative enzymes.

Overarching results from this study stress the importance of integrating the gut microbiome into the study of carcinogen metabolism and carcinogenesis. With the ever omnipresent threat and buildup of carcinogens within the industrialized 
environment and the resulting inevitable daily exposure of the colon to these compounds, a more integrated approach to the prevention of cancer is needed. Future studies assessing potential synergy that may arise when dysbiosis of the gut microbiome is combined with exposure to environmental carcinogens such as $\mathrm{B}[\mathrm{a}] \mathrm{P}$ could lead to a better understanding and a potential explanation for the current upward tick in colorectal cancer cases.

\section{Abbreviations}

$\mathrm{B}[\mathrm{a}] \mathrm{P}$ : Benzo[a]pyrene, CRC: colorectal cancer, PAHs: polycyclic aromatic hydrocarbons, Anthos: native anthocyanidins mixture from billberry, CYP: cytochrome P450, mEH: microsomal epoxide hydrolase, BPDE: benzo[a]pyrene7,8-diol-9,10-epoxide, GST: glutathione s-transferase, UGT: uridine 5'diphospho-glucuronosyltransferase, SULT: sulfotransferase, AhR: aryl hydrocarbon receptor, AhRR: aryl hydrocarbon receptor repressor, PXR: pregnane X receptor, UDPGT: Glucuronosyltransferase, APC: adenomatous polyposis coli, COX-2: cyclo-oxygenase-2, Cy: cyanidin, Dp: delphinidin, Pt: petunidin, Mv: malvidin, Pe: peonidin, HPLC: high performance liquid chromatography, PDA: photodiode array detector, UV: ultraviolet, ETBF: enterotoxigenic Bacteriodes fragilis, BFT: $B$. fragilis toxin. 
CHAPTER 4:

MODULATION OF COLON INFLAMMATORY ENVIRONMENT BY BILBERRY-DERIVED

ANTHOCYANIDINS

\section{Introduction}

A recent report on the incidence of colorectal cancer (CRC) has uncovered an alarming increase in the rate in individuals under the age of 40 . Interestingly, this increase was only observed for cases in the distal colon and rectum, which had previously been declining [12]. Although the cause for this shift has yet to be identified, one particularly compelling presumption would be to link an updated version of Strachan's hygiene hypothesis [172]. This hypothesis associates the rise in atopic diseases such as food allergies, asthma, anaphylaxis, allergic rhinitis and atopic dermatitis and disorders of the immune system e.g., inflammatory bowel disease (IBD) and type 1 diabetes (T1D) in the younger generations to the increase in "hygienic" environments in the "developed" world since the 1980's. During this time, great strides have been made in the fight against infectious diseases due to increased vaccination rates, use of antibiotics and sanitation practices [173]. However, although the hygiene hypothesis makes a compelling case, additional changes such as alterations in diet, environmental carcinogens, exposure to parasites, increase in caesarean sections etc. have occurred concurrently during this time period. The updated hypothesis which now 
targets promoting a healthy microbiome while maintaining stringent standards for avoiding infectious diseases has taken on a variety of names such as microbiome depletion hypothesis, old friends hypothesis, microbial diversity hypothesis and targeted hygiene hypothesis [174].

\section{Bacteria and evasion of immune response}

Traditionally, the cause for the development of CRC has been attributed to the presence of genetic mutations. However, the role of the gut microbiome in the development of cancer, with a special emphasis on CRC, has been gaining everincreasing traction within the scientific community over the past 10 years. Specifically, findings link the dysbiosis of the gut microbiome and the resulting inflammation to genotoxicity and ultimately carcinogenesis. Various mechanisms by which these "bad" bacteria take root and evade the immune system have been hypothesized and include the ability of toxin-producing bacteria such as $H$. pylori and $B$. fragilis to evade the normal host response. This was found to be in part due to their ability to elicite a Th2 over Th1 response caused by their lipid A structural differences which fail to activate toll like receptor 4 (TLR4), but activate toll like receptor 2 (TLR2) [175]. Originally described by Mosmann et al., the T helper (Th) cell balance between Th1 and Th2 cell subtypes, Th2 dominated imbalances, which lead to increases in IL-4, IL-5 and IL-13, were found to lead to allergen sensitization. In contrast, imbalances favoring Th1 type responses, which lead to increases in IFN- $y$, were shown to suppress Th2 and ultimately lead to the inhibition of atopic immunopathology. 
A more recently identified Th subtype is Th17. Under normal functioning conditions, Th17 cells aid in defending the host against extracellular pathogens. This is accomplished by aiding in the recruitment of macrophages and neutrophils to the infected tissue. However, when dysregulated Th17 cells can lead to chronic inflammatory diseases such as multiple sclerosis, rheumatoid arthritis, psoriasis, asthma and inflammatory bowel disease [176]. Recent studies have linked the environmental factors such as diet and intestinal microbiota to this dysregulation of cytokine regulation ultimately spurring autoimmunity and disease [177]. Interestingly, studies have linked diets high in trans fatty acids with increased expression of $\mathrm{IL}-17$ and RORyt expression which are characteristic of a Th17 response and intestinal inflammation in DSS treated mice [178]. Additionally, a diet high in salt was linked with promotion of both Th17 differentiation and the induction of autoimmunity [179].

One particular strain of bacteria that has gained attention for both its relevance in clinical populations for CRC, FAP and diarrheal disease is Enterotoxigenic Bacteriodes fragilis (ETBF). ETBF bacteria are a subset of $B$. fragilis bacteria that secrete the metalloprotease toxin, $B$. fragilis toxin (BFT). This $20 \mathrm{kDa}$ zincdependent metalloprotease leads to the cleavage of E-cadherin thus increasing permeability and exposing the submucosa of the colon to bacterial antigens from the lumen. Additionally, the tumorigenesis induced by ETBF bacteria has been found to be driven by Th17 response and increased STAT3 activity.

Like many biological processes, the balance between these competing pathways is crucial in maintaining an optimal disease-free state. Excess of any one of 
these pathways under certain conditions can lead to a variety of diseases such as asthma, rheumatoid arthritis, colitis, and cancer $[180,181]$. It has also been shown that by depleting the Tregs in ETBF-colonized C57BL/6 FOXP3(DTR) mice, there was an effective shift toward enhanced colitis, but reduced tumorigenesis. This was attributed to the shift in the mucosal cytokine profile from IL17 to IFNy [182]. A variety of factors have been found to influence Th17 cell differentiation. These factors include but are not limited to the following: influence of Treg, PD-L1, AhR, COX-2/PGE2 [183].

\section{Programmed death-ligand 1 (PD-L1)}

A number of promising immunotherapeutic treatments such as ipilimumab (antiCTLA-4 therapy), pembrolizumab (anti-PD-1 therapy), nivolumab (anti-PD-1 therapy), atezolizumab (anti-PD-L1 therapy), avelumab (anti-PD-L1 therapy), and durvalumab (anti-PD-L1 therapy) have recently been developed and successfully applied to the treatment of several different types of cancer such as melanoma, Hodgkin lymphoma, urothelial carcinoma and non-small cell lung cancer. Of these immune therapies, one of the most common targets is the programmed death ligand 1 (PD-L1) and its receptor programmed death 1 (PD-1) receptor. The PD-1 receptor is expressed by $T$ lymphocytes, B, natural killer $T$, and Treg cells, where it functions as an inhibitory receptor which effectively diminishes the immune response via down-regulation and promotes self-tolerance by suppressing T cell inflammatory activity. Under normal functioning conditions this helps prevent autoimmune disease. However, this mechanism is often redirected 
by cancer cells, which highly express the PD-1 ligand, PD-L1, in order to prevent the immune system recognition and attack.

With the recent rise in use of the checkpoint inhibitors such as anti-PD1 and antiCTLA-4 therapies, side effects classified as immune-related adverse events (irAEs) ranging from pneumonitis and hepatitis to colitis have arisen [184]. More specifically, it has been found that a new inflammatory bowel disease phenotype that shares traits with both ulcerative colitis (UC) and crohn's disease (CD) can occur with use of these therapies [184]. These side effects are not surprising considering that several studies have shown that polymorphisms in these proteins are associated with autoimmune diseases such as type 1 diabetes mellitus (T1DM), systemic lupus erythematosus (SLE) and rheumatoid arthritis (RA) [185]. Interestingly, upregulation of PD-L1 has been found to restrain Th17 cell differentiation [186]. As noted previously, Th17 is one of the main drivers by which ETBF bacteria drives inflammation-induced colon carcinogenesis [138]. Therefore, although PD-L1 has a deleterious role when expressed in the tumor microenvironment, a more careful consideration of the endogenous role of PD-L1 in normal colon tissue must be addressed.

\section{Role of COX-2 in PD-L1 expression and Th17 differentiation}

Even though COX-2 was originally identified in 1989 by Needleman and coworkers, COX-2 and COX-1 have been anti-inflammatory targets from the ancient time - since $1500 \mathrm{BC}$ [187]. COX-2 is typically expressed only under conditions where prostaglandins are upregulated which occurs during inflammation and have thus been a key target for research relating to the 
treatment and prevention of diseases such as rheumatoid arthritis, osteoarthritis, and cancers of the prostate, breast, lung and colon [188]. In addition to the more traditional therapeutic applications of the COX-2/PGE2 pathway, recent research has highlighted the influence of more mechanistic role of this pathway on PD-L1 expression and Th17 development. For instance, it has also been shown that tumor cells can induce PD-L1 expression in macrophages and myeloid-derived suppressor cells by altering PGE2 metabolism in hematopoietic cells and that COX-2 inhibition could help to regulate these changes in PD-L1 expression [189]. Furthermore, COX-2 expression was also found to be positively correlated with PD-L1 expression in melanoma and inhibition of COX-2 by celecoxib effectively down-regulated PD-L1 expression in two melanoma cell lines [190]. Interestingly, COX-2 expression was found to increase expression of IL-4 and IDO but was also found to be inversely related to expression of IFN- $y$ in fibrosarcomas [191]. Thus, under circumstances of high local expression of COX-2 it is thought that this induces immunosuppression and ultimately impairs the $T$ cell effector functions which are mediated by IFN-ץ [191].

In addition to influencing PD-L1 expression, the PGE2/COX2 pathway has also been found to influence Th17 differentiation. PGE2 has been shown to promote the differentiation and pro-inflammatory functioning of Th17 cells in both mice and humans by inducing EP2 and EP4 signaling altering IFN- $\gamma$, and IL-10 production [192]. PGE2 was also found to be required for IL-17 production in the presence of APC cells [192]. With the ever-expanding mechanistic influence of 
this pathway, it appears that the "gold-standard" for inflammation and the prevention thereof still has a few mysteries that have yet to be unraveled.

With the above factors in mind, it is easy for one to grasp how complex the balance in host immune response can be. Given the deleterious role of Th17 in carcinogenesis induced by ETBF bacteria residing in the colon, identifying a method by which this imbalance in T helper cell signaling can be restored in order for the host to identify and effectively rid itself of ETBF, while limiting harm to itself, would be an ideal scenario.

\section{Modulation of gut microbiome dialogue with host}

An important factor that should be considered when addressing dysbiosis of the gut microbiome is the role that factors ranging from diet, whether an individual was breast fed [193], antibiotic usage or whether they were exposed to environmental carcinogens should be considered regarding the formation of dysbiosis. For instance, the influence of dietary intake of refined sugars has been found to increase levels of $C$. difficile and $C$. perringens which has been linked to IBD[194]. Furthermore, individuals who consumed a more plant-based agrarian diet versus Western-style diet rich in meat and fat were found to have altered microbiomes $[195,196]$. With the influence of dietary intake in mind, the potential role of particular phytochemicals on the gut microbiome and its interaction with the host is a key factor that needs to be investigated. 
Anthocyanin-rich foods have been a dietary staple seemingly since the dawn of time. Known for their characteristically-rich purple, blue and red hues that canvas/envelop a variety of fruits, vegetables, grains and flowers, anthocyanins have been attributed to providing a variety of health promoting properties such as activities including anti-inflammatory [94], chemopreventive and chemotherapeutic $[86,130]$, obesity control, cardiovascular disease prevention, and alleviation of the symptoms of diabetes [131].

As work presented in the preceding chapters has shown, Anthos works on a variety of pathways including, phosphorylation status of Src and EGFR and modulation of phase $\mathrm{I} / \mathrm{II}$ enzyme expression in and ETBF bacterially-driven model of $\mathrm{CRC}$ in $\mathrm{Apc}^{\mathrm{Min} /+}$ mice. For this series of studies, we turned our attention to the influence of Anthos on the inflammatory response to ETBF bacteria both within the tumor and adjacent normal microenvironments and the influence of Anthos dose on colon tumor number and tumor grade.

\section{Methods}

\section{Anthos Isolation and Purity}

The native bilberry Anthos mixture that was generously provided by $3 \mathrm{P}$ Biotechnologies (Louisville, KY) was further enriched using C18 Sep-Pak cartridges (Waters, Milford, MA) and eluted in acidified $(0.1 \% \mathrm{HCl})$ ethanol. The enriched extract was then dried using a Savant SC210A Speed-Vac (ThermoFisher Scientific, Waltham, MA) and stored at $-20^{\circ} \mathrm{C}$, under argon. Purity was determined by HPLC-PDA-UV. Briefly, $15 \mu \mathrm{l}$ samples were analyzed using a Shimadzu Premier C18 reverse-phase column (250x4.6 mm i.d., $5 \mu \mathrm{m}$ ). 
Mobile phase A was composed of water: formic acid: acetonitrile (87:10:3) and mobile phase B was composed of water: formic acid: acetonitrile (40:10:50). The flow rate was $0.6 \mathrm{ml} / \mathrm{min}$ and the gradient condition was $0-5 \min 5 \% \mathrm{~B} ; 5-15 \mathrm{~min}$ $15 \%$ B; $15-20 \min 25 \%$ B; $20-30$ min $35 \%$ B; $30-40 \min 45 \%$ B; $40-45 \min 100 \%$ B; $45-50$ min $5 \%$ B. Detection of Anthos was at $520 \mathrm{~nm}$ by PDA-UV and total Anthos concentration was calculated using a standard curve. The reference anthocyanidins were purchased from Cayman Chemical Company (Ann Arbor, $\mathrm{MI})$.

\section{In vivo Anthos dose escalation study}

Animal experiments were performed in agreement with an approved protocol by the Institutional Animal Care and Use Committee at the University of Louisville. Breeding colonies were established in collaboration with Dr. Nejat K. Egilmez's lab [138] at the University of Louisville using C57BL/6J Min/+ $\left(\mathrm{Apc}^{\mathrm{Min} /+}\right)$ mice that were originally attained from Jackson Laboratories (Bar Harbour, ME). Mice were genotyped for the APC mutation using PCR according to the protocol established by Jackson Laboratories. Mice were fed a standard chow diet and received water ad libitum and were maintained on a standard light/dark cycle for the duration of the study. At 5-6 weeks of age, animals were administered antibiotic. Four days later the animals were administered ETBF to promote tumorigenesis and one week following ETBF inoculation, animals began their respective treatment regimen. The dose escalation study featured three doses, namely, low (8.6 $\mathrm{mg} / \mathrm{kg} /$ day, i.e. $60 \mathrm{mg} / \mathrm{kg} / \mathrm{wk}$ ), medium (17.1 mg/kg/day, i.e. $120 \mathrm{mg} / \mathrm{kg} / \mathrm{wk}$ ) and high (34.3 mg/kg/day, i.e. $240 \mathrm{mg} / \mathrm{kg} / \mathrm{wk})$ dosages. Male and female Apc $\mathrm{Min} /+^{+}$ 
mice ( $n=5$ combined) were randomly assigned to each treatment or vehicle control group and were treated via oral gavage three times per week for 4 weeks. Animals were culled in the fed state at 12 weeks, colon tumors were counted and tissues were harvested.

\section{Pathology}

Following excision, colon tumor and mesenteric lymph tissue were immediately fixed in a solution of $10 \%$ formalin. After 48 hours, samples were transferred to a solution of $70 \%$ ethanol. Samples were embedded in paraffin and sectioned by the pathology core research laboratory, department of pathology and laboratory medicine at the University of Louisville. Slides were embedded in paraffin, sectioned and stained with H\&E and subsequently evaluated by pathologist, Dr. Mostafa Fraig. Digital slides were captured using a Leica Aperio digital pathology slide scanner (Leica Biosystems, Wetzlar, Germany).

\section{Western-blot analysis}

For western-blot analysis, $40 \mu \mathrm{g}$ of protein from in vitro and in vivo tissue lysates was by $4-12 \%$ polyacrylamimide - SDS gel electrophoresis and electrotransferred to polyvinylidene difluoride membranes by semi-dry transfer (Biorad Trans-blot SD, Hercules, CA). Blots were blocked with 4\% dry powder milk or BSA for $1 \mathrm{~h}$ and then incubated with primary antibodies $\mathrm{p} 21$, IFN- $\gamma$, TLR4, phosphor-p38, phosphoVEGF, IFN- $\beta$, VEGF and COX-2 which were all acquired from Santa Cruz Biotech (Santa Cruz, CA) and PD-L1 which was acquired from Proteintech (Rosemont, IL) at $4^{\circ} \mathrm{C}$ overnight and secondary antibodies (Santa Cruz Biotech, Santa Cruz, CA). conjugated to peroxidase for 1 
$\mathrm{h}$ at room temperature. Blots were then developed with an ECL detection system. Densitometric analysis was then performed using ImageJ 1.x software [134].

\section{Results}

\section{Effect of Anthos on tumor inhibition}

Following our previous findings, we pursued a detailed dose-escalation study to determine the influence of Anthos dosage on tumor count. Three doses were tested - low (8.6 mg/kg/day, i.e. $60 \mathrm{mg} / \mathrm{kg} / \mathrm{wk})$, medium (17.1 mg/kg/day, i.e. 120 $\mathrm{mg} / \mathrm{kg} / \mathrm{wk}$ ) and high (34.3 mg/kg/day, i.e. $240 \mathrm{mg} / \mathrm{kg} / \mathrm{wk})$. Controls included a vehicle control and an $\mathrm{Apc}^{\mathrm{Min} /+}$ mice without ETBF bacteria control. Results from this study (Figure 23) showed a clear significant dose- dependent decrease in colon tumor counts in Anthos-treated groups. Significant differences in tumor counts were noted between control and low dose animals $(P=0.003)$, control and medium dose animals $(P=0.0004)$, control and high dose animals $(P<0.0001)$. Additionally, significant inter-treatment group differences were noted for medium and high doses $(P=0.0001)$ and low and high doses $(P<0.0001)$. The difference between low and medium treatment groups was not statistically significant $(P=0.054)$. No significant difference in tumor number was noted between male and female animals within any treatment group. 


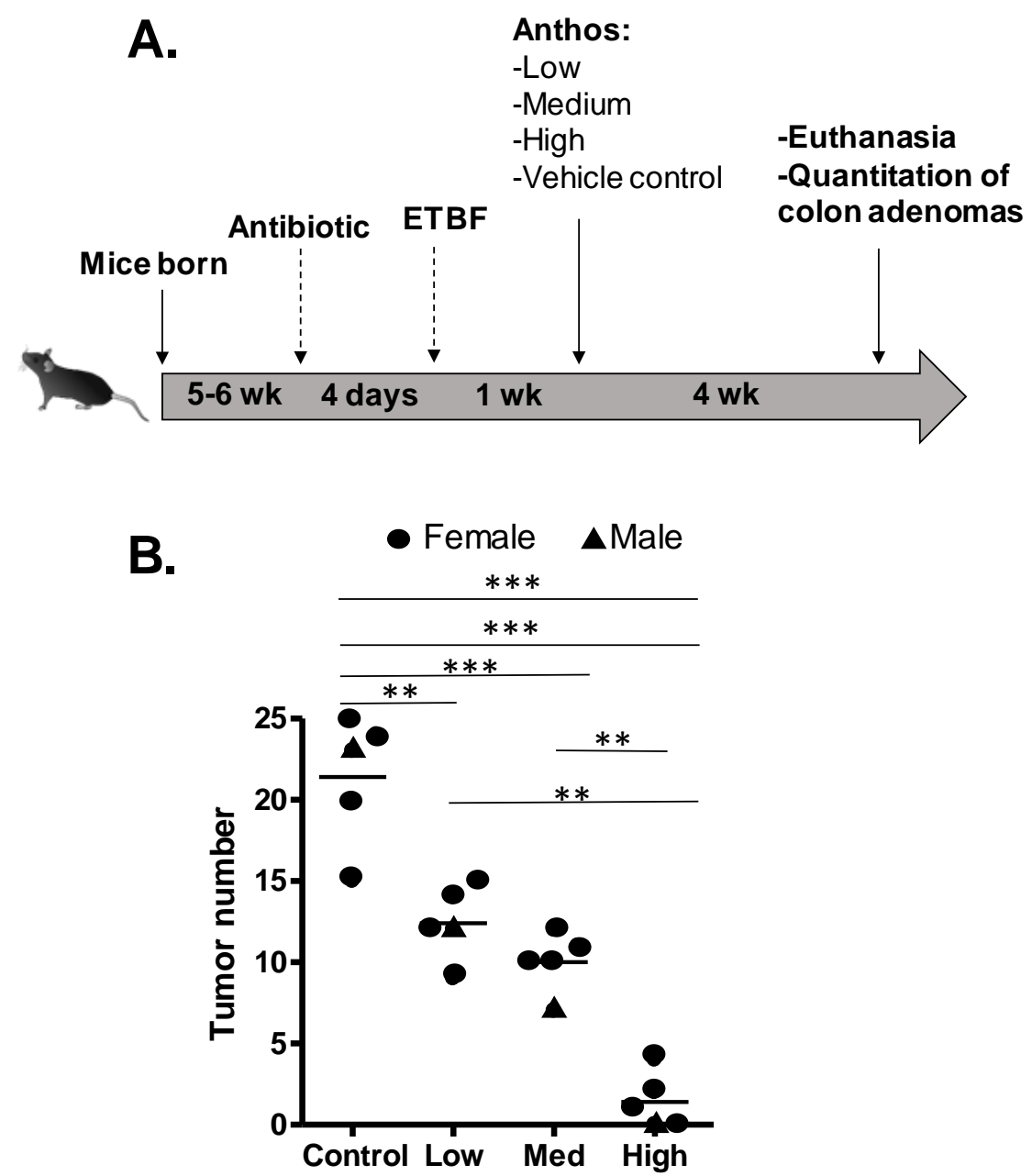

Figure 23. In vivo Anthos dose escalation study: $\mathrm{Apc}^{\mathrm{Min} /+}$ mice inoculated with ETBF were treated via oral gavage, three doses a week for 4 weeks with low (8.6 mg/kg), medium $(17.1 \mathrm{mg} / \mathrm{kg})$ and high $(34.3 \mathrm{mg} / \mathrm{kg})$ dosages or vehicle control. (A) Study overview. (B) tumor numbers. Black triangles represent males and black circles represent females. ${ }^{* *}$ signifies $P<0.001,{ }^{* *}$ signifies $P<0.01$, and * signifies $P<0.05$. 


\begin{tabular}{|c|c|c|c|c|c|}
\hline \multirow{2}{*}{$\begin{array}{c}\text { Animal } \\
\text { number }\end{array}$} & \multirow{2}{*}{ Gender } & \multicolumn{5}{|c|}{ Dosage group } \\
\cline { 3 - 6 } & & Control & Low & Medium & High \\
\hline 1 & $\mathrm{~F}$ & 20 & 9 & 10 & 4 \\
\hline 2 & $\mathrm{~F}$ & 25 & 12 & 12 & 3 \\
\hline 3 & $\mathrm{~F}$ & 24 & 15 & 10 & 2 \\
\hline 4 & $\mathrm{~F}$ & 15 & 14 & 11 & 1 \\
\hline 5 & $\mathrm{M}$ & 23 & 12 & 7 & 5 \\
\hline \multicolumn{2}{|l}{ Avg tumor number } & 21 & 12 & 10 & 3 \\
\hline
\end{tabular}

Table 5. Tumor numbers for in vivo Anthos dose escalation study 

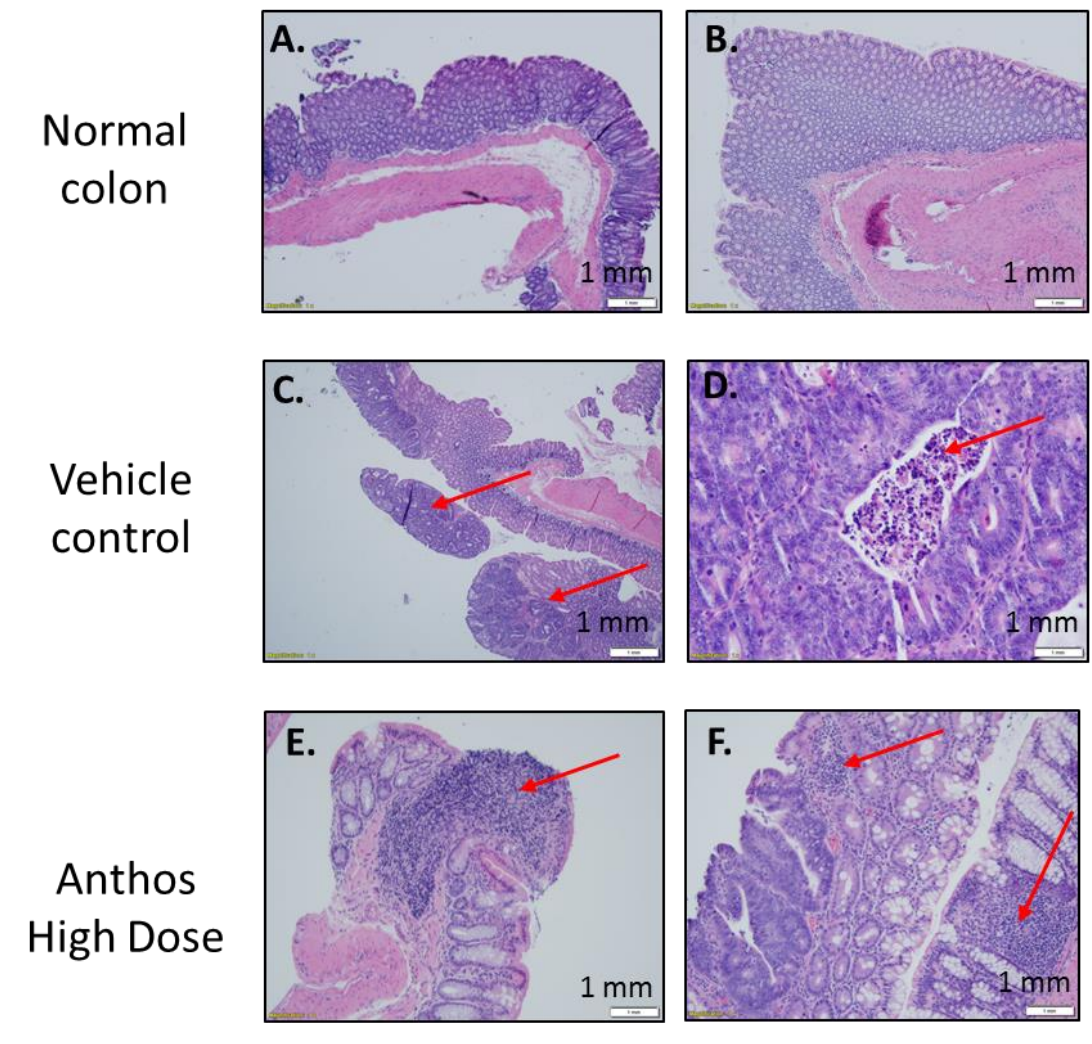

Figure 24. Anthos-treated $\mathrm{Apc}^{\mathrm{Min} /+}$ mice inoculated with ETBF featured fewer, more well differentiated tumors, with immune response:

Representative photographs of histological analysis of colon adjacent normal and tumor samples taken from Anthos-treated (4E, 4F) and vehicle control (4C, 4D) as compared to normal colon tissue (4A, 4B). Paraffin sections of colons were stained with H\&E. Arrows in 4C and 4D highlight representative tumors (4C) and necrotic patches (4D) observed in vehicle control mice. Arrows in 4E and 4F represent lymphoid aggregates adjacent to normal (4E) and tumor and normal colon tissue (4F). No lymphoid aggregates were observed in vehicle control mice. Scale bars $=1 \mathrm{~mm}$ for all figure panels. 


\section{Mechanism}

\section{In vivo look at inflammation}

Building upon the findings regarding the influence of Anthos on the phosphorylation status of Src and EGFR, STAT3, STAT5 and downstream expression of key targets cyclin D1 and cyclin D2 that were presented in the previous chapter, we next sought to determine the influence of Anthos treatment on the inflammatory tumor and adjacent normal microenvironments in the same ETBF Apc ${ }^{\mathrm{Min} /+}$ mouse model. Results from our western blot analysis (Figures 2527) of the inflammation pathways associated with CRC and immune checkpoint function showed significant increases in the expression of IFN-y $(P=0.04)$, phospho-p38 ( $P=0.03)$, and levels of TLR-4 $(P=0.01)$, and significant decreases in the expression of COX-2 $(P=0.001)$, in adjacent normal tissue. In the tumor tissue microenvironment, decreased levels of TLR-4 $(P=0.01)$ were noted along with decreased expression of COX-2 $(P=0.03)$, and increased expression of p21 $(P=0.03)$ in Anthos vehicle control animals. Interestingly, expression of the key immune checkpoint protein, PD-L1, was significantly increased in adjacent normal tissue $(P=0.01)$ of animals treated with Anthos versus vehicle control and significantly decreased in tumor tissue in these same Anthos versus control animals $(P=0.005)$. 
Adjacent Normal

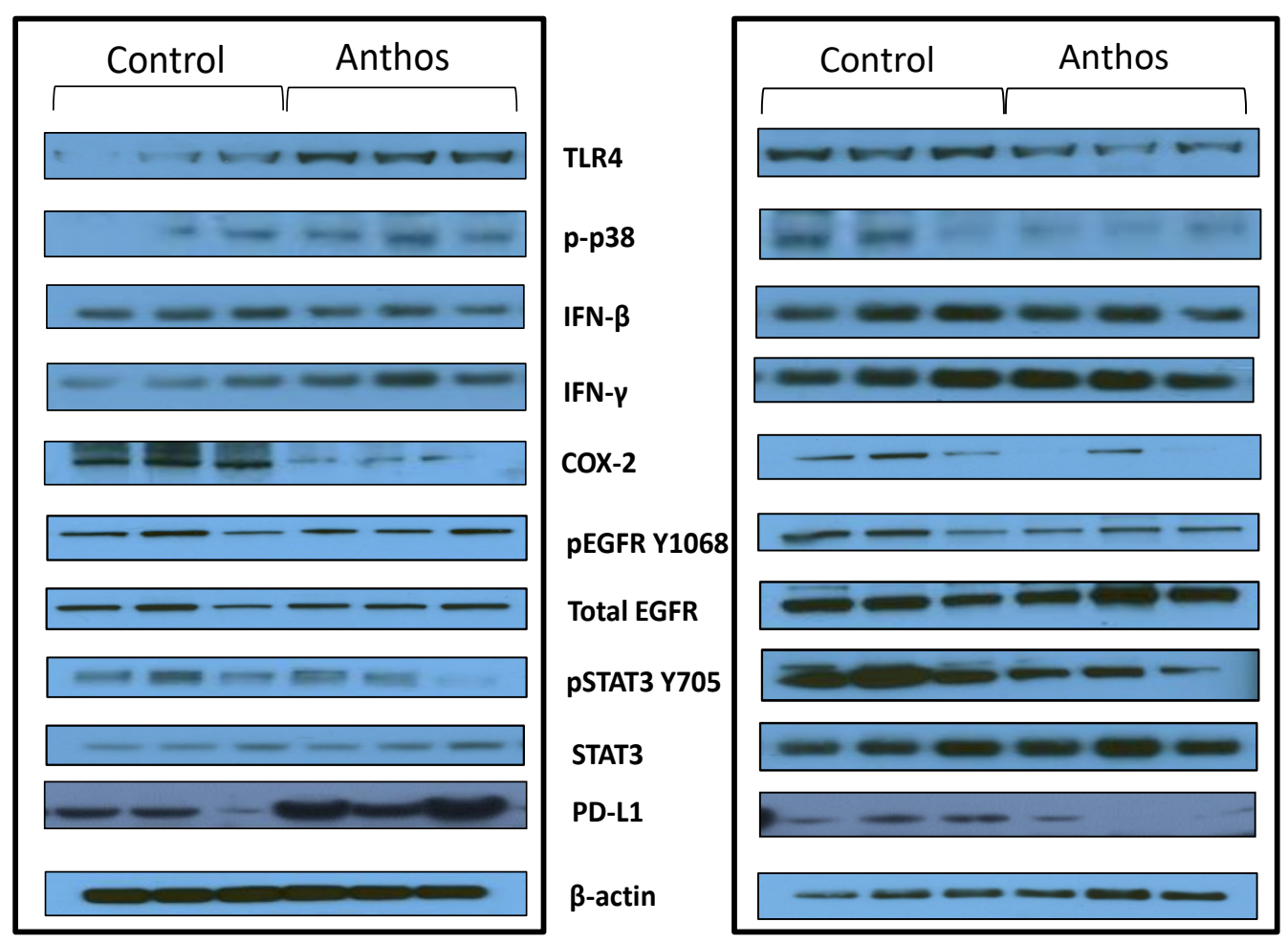

Figure 25. In vivo changes in PD-L1 related expression and upstream pathways following treatment with Anthos: changes in the phosphorylation and expression of EGFR along with corresponding downstream pathway targets including STAT3, PD-L1, COX-2 and IFN- $\gamma$ related pathways in a) adjacent normal and b) tumor colon tissue taken from ETBF-inoculated $\mathrm{Apc}^{\mathrm{Min} /+}$ mice after treatment with Anthos or vehicle control as assessed using western blot analysis with $\beta$-actin loading control. 


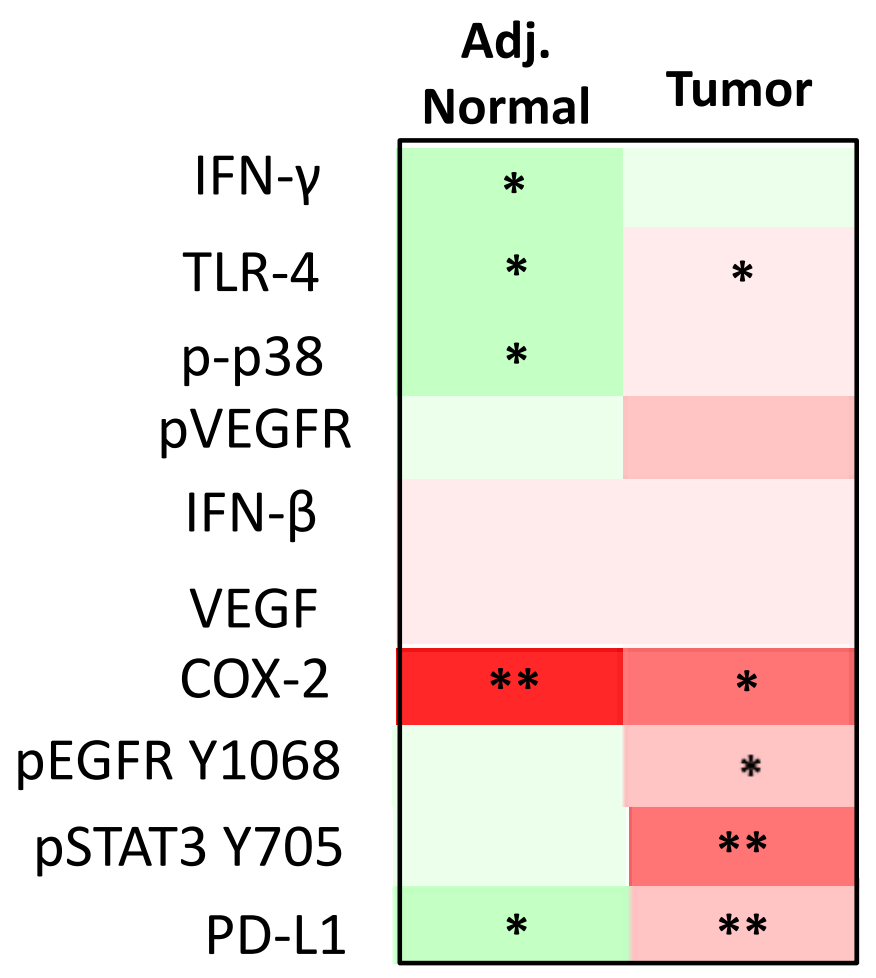

Significance level attained for comparison of average Anthos to control sample expression/phosphorylation

$$
\begin{aligned}
& \mathrm{P}<0.05^{*} \\
& \mathrm{P}<0.01^{* *} \\
& \mathrm{P}<0.001^{* * *}
\end{aligned}
$$

Fold change

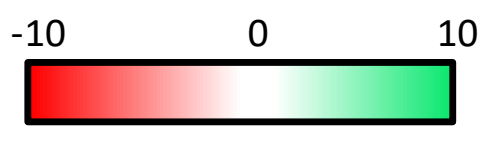

Figure 26. Representative heatmap for in vivo changes in PD-L1 related expression and upstream pathways following treatment with Anthos: representative heat map legend depicts the average fold change and significance level in either phosphorylation status or expression levels between Anthos treated and vehicle control animals within either adjacent normal $(n=3)$ or tumor colon tissue $(n=3)$. 
Table 6. Raw densitometry values calculated for PD-L1/inflammation pathway western blot analysis: values determined using ImageJ software

\begin{tabular}{|c|c|c|c|c|c|c|}
\hline \multirow{2}{*}{ Target } & \multicolumn{3}{|c|}{ Adjacent Normal } & \multicolumn{3}{c|}{ Tumor } \\
\cline { 2 - 7 } & Control & Anthos & $\begin{array}{c}\text { Fold } \\
\text { change }\end{array}$ & Control & Anthos & $\begin{array}{c}\text { Fold } \\
\text { change }\end{array}$ \\
\hline EGFR & 18661 & 20531 & 1 & 18388 & 20599 & 1 \\
\hline $\begin{array}{c}\text { pEGFR } \\
\text { (Y1068) }\end{array}$ & 19771 & 17469 & -1 & 25990 & 12493 & -2 \\
\hline pSTAT3 & 15938 & 11067 & -1 & 25354 & 5274 & -5 \\
\hline STAT3 & 5088 & 7000 & 1 & 10291 & 8208 & -1 \\
\hline COX-2 & 19808 & 2687 & -7 & 18224 & 4358 & -4 \\
\hline TLR-4 & 3739 & 10338 & 3 & 10756 & 7058 & -2 \\
\hline p-p38 & 3069 & 7907 & 3 & 9136 & 5246 & -2 \\
\hline IFN- $\beta$ & 6487 & 5833 & -1 & 10840 & 9090 & -1 \\
\hline IFN-Y & 2898 & 5816 & 2 & 9955 & 11484 & 1 \\
\hline PD-L1 & 23650 & 62231 & 3 & 20351 & 6728 & -3 \\
\hline
\end{tabular}



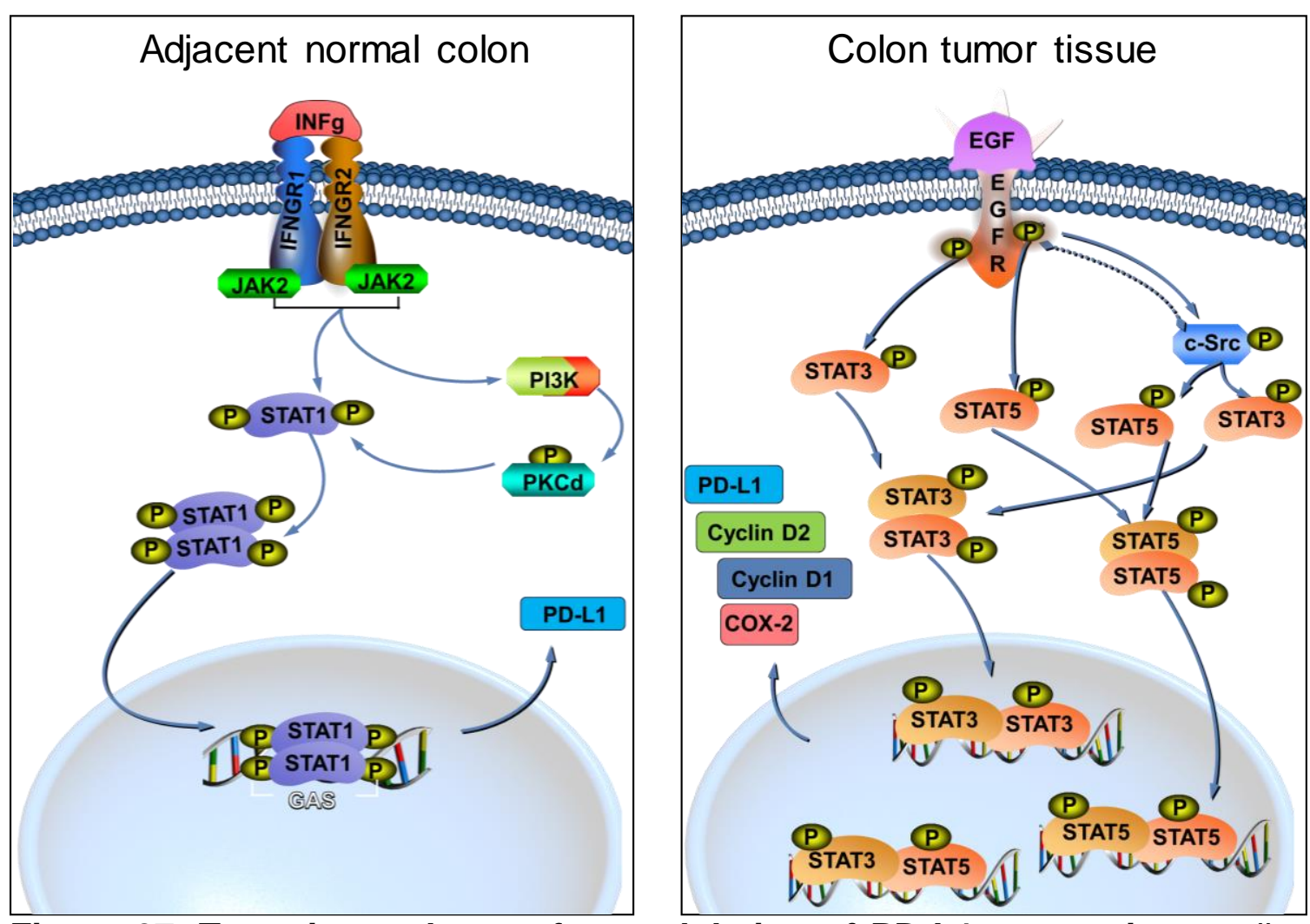

Figure 27. Tentative pathways for modulation of PD-L1 expression: outline of EGFR and IFN- $\gamma$ driven expression of PD-L1 proposed for adjacent normal and tumor tissue samples. Pathway is a modified version of the Qiagen EGFR and IFNy signaling pathways. 
A.

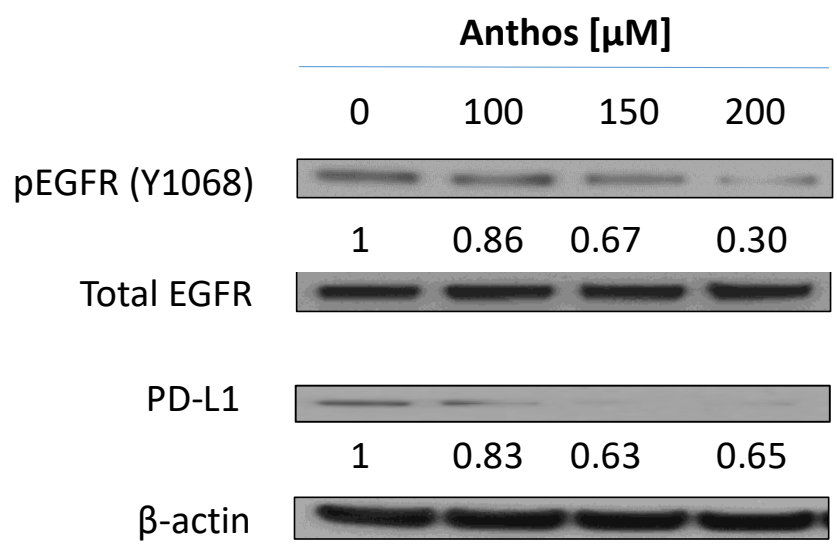

B.

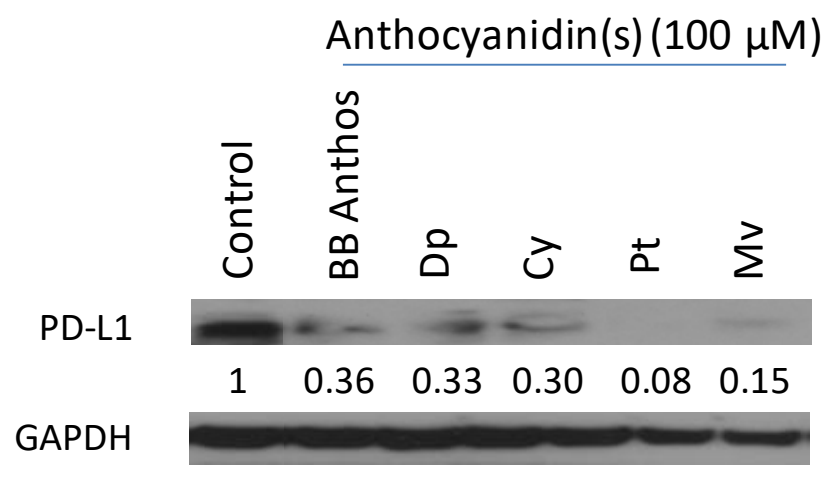

Figure 28. In vitro changes in PD-L1 expression and related EGFR phosphorylation status following Bilberry-derived Anthos and anthocyanidin treatment: a) dose dependent changes in the phosphorylation status of EGFR (Y1068) and downstream PD-L1 expression in HT-29 cells treated with varying concentrations of bilberry-derived Anthos; b) influence of indicated individual anthocyanidins $(100 \mu \mathrm{M})$ on PD-L1 expression in HT-29 colon cancer cells as assessed using western blot analysis with $\beta$-actin and GAPDH loading controls. 


\section{In vitro impact of Anthos and individual anthocyanidins on PD-L1 expression}

Following the promising findings in vivo, we wanted to confirm that Anthos treatment decreased PD-L1 expression in vitro in established human colon cancer cell lines, HT-29 and HCT-116. Results from our look at PD-L1 expression in these two colon cancer cell lines showed that Anthos treatment led to dose-dependent decreases in PD-L1 expression (Figure 28). Follow-up work assessing the influence of each individual anthocyanidin and comparing it to the native Anthos mixture showed that the most impressive reduction in PD-L1 expression was achieved by petunidin (Figure 28$)$ at the same dosage $(100 \mu \mathrm{M})$.

\section{Discussion}

In the previous chapters (Chapters 2 and 3 , we identified Anthos as a promising chemopreventive agent against colorectal tumor development in an $\mathrm{Apc}^{\mathrm{Min} /+}$ ETBF mouse CRC model. We showed that Anthos functions by decreasing the phosphorylation of Src and EGFR thereby modulating phosphorylation status or expression of key downstream players including STAT3, STAT5, COX-2, Cyclin D1 and Cyclin D2 in chapter 2. We also showed that Anthos treatment led to modulation of the metabolic detoxification pathways in chapter 3 . In this chapter we therefore sought to determine the influence of Anthos treatment on the inflammatory environment in colon adjacent normal and

tumor tissue in an $\mathrm{Apc}^{\mathrm{Min} /+}$ ETBF model. We also sought to identify the doseresponse range for Anthos treatment in vivo. 
ETBF bacteria is the prototypical bacteria posited to serve as an "alpha-bug" which is both directly pro-oncogenic and is also able to influence and remodel the colonic microbiome in such a way that ultimately further promotes its own ability to induce mucosal immune responses and changes within the colon epithelial cells ultimately resulting in colon cancer [24]. ETBF bacteria are a subclass of the $B$. fragilis bacteria that secrete the metalloprotease, $B$. fragilis toxin, which is structurally related to the tetanus and diphtheria toxins. Ultimately, BFT leads to the stimulation of E-cadherin cleavage thereby leading to increased colon permeability, inflammation and $\beta$-catenin nuclear signaling [26]. It has also been previously shown that Treg depletion leads to the mitigation of the Th17 response to ETBF bacteria in favor of a Th1 response [182]. Results from this series of studies suggests that Anthos treatment leads to increased levels of TLR4 and secreted IFN- $\gamma$ and decreased expression of COX-2 in adjacent normal colon tissue and increased expression of p21 and decreased levels of TLR-4 in colon tumor tissue. Interestingly, we also showed that PD-L1 expression was significantly upregulated in normal colon tissue and significantly decreased in colon tumor tissue samples taken from Anthos treated animals. The findings presented in chapter 2, which show that Anthos alter the phosphorylation status of EGFR and Src as well as downstream STAT3 in colon tumor tissues taken from the ETBF treated $\mathrm{Apc}^{\mathrm{Min} /+}$ mice support the decrease in PD-L1 expression noted in the same colon tumor tissue. This modulatory specificity, which in effect, differentially modulates PD-L1 levels in colon tumor over normal tissue provides an interesting application for Anthos treatment to not only the 
prevention of offsite toxicities associated with the current standard of care, but also serve as a potential modulator of PD-L1 over-expression specific to colon tumor tissue.

In the previous chapters we have shown that Anthos treatment at $120 \mathrm{mg} / \mathrm{kg} / \mathrm{wk}$ led to significant reductions in the number of colon tumors when compared to vehicle control animals. In order to gain a better understanding of the influence of Anthos dosage on tumor number we conducted a dose escalation study with Anthos in $\mathrm{Apc}^{\mathrm{Min} /+}$ mice treated with ETBF bacteria. Results from this study showed a significant dose dependent decrease in tumors for all treatment groups. Treatment at the highest dose of $240 \mathrm{mg} / \mathrm{kg} / \mathrm{wk}$ led to over an $86 \%$ reduction in colon tumor development $(\mathrm{P}<0.001)$. The appearance of tumors in all treatment groups were also much flatter and smaller in size than the tumors from vehicle control animals.

The influence of Anthos on the inflammatory environment identified in this chapter highlights the potential influence of these factors under conditions of environmental carcinogen exposure. It has been shown that polycyclic aromatic hydrocarbon (PAH) exposure induces Th17 cell differentiation and IL-17 secretion in T cells in an AhR dependent mechanism [197]. As presented in previous chapters, along with this chapter ETBF bacteria and benzo[a]pyrene $(\mathrm{B}[\mathrm{a}] \mathrm{P})$ were shown to also lead to increased levels of $\mathrm{AhR}$ expression in vivo and in vitro, respectively. With this in mind, one could extend these findings 
to posit that the recent increases in autoimmune diseases such as IBD and T1D could potentially be associated with dual environmental insults such as carcinogen exposure and microbiome dysbiosis.

The effect of the combined insult of environmental carcinogens such as PAHs and microbiome dysbiosis is still essentially unknown. Given the increasing industrialization of our environment and exposure to carcinogens such as $\mathrm{B}[\mathrm{a}] \mathrm{P}$, additional research assessing whether a synergistic carcinogenic dialogue exists between the ETBF bacteria and the ubiquitous environmental carcinogen, $\mathrm{B}[\mathrm{a}] \mathrm{P}$, is a key area of future research that is needed. Additionally, further investigation into the exact nature of Anthos upregulation of $A h R R$ is needed. Classically, AhRR expression is upregulated by AhR. Previous studies have found that different AhR ligands differentially induce distinctly different, ligand specific AhR dependent gene products [198]. AhR has also recently been identified as a player in intestinal barrier function, homeostasis, and intestinal immune cells [199]. Interestingly, it has also been shown that activation of AhR by a high affinity ligand during Th17 development led to significant increases in not only the proportion of $\mathrm{T}$ cells but also their production of cytokines [200]. This is particularly interesting considering our findings in chapter 3, which showed how Anthos treatment influences the AhR pathway, in relation to its role in altering phase $\mathrm{I} / \mathrm{Il}$ enzyme expression. 
Although it was not in the scope of this chapter, the influence of Anthos on the gut microbiome is the next area of investigation that needs to be addressed. Although our preliminary look at the influence of Anthos on the total ETBF bacteria population in vitro suggests that Anthos does not directly influence the total CFUs of the ETBF bacteria in the absence of a cellular/immunological environment (unpublished data). However, the results presented here suggest that Anthos most likely influences the immunological balance in the colon adjacent normal and tumor microenvironment and may therefore, by extension, influence the host immune response against this toxigenic bacteria. Future work assessing the microbiomes of Anthos and vehicle treated animals using fecal and mucosal biopsy samples should aid in elucidating the influence of Anthos treatment on gut microbiota. Additionally, kinetic studies assessing the modulation of $\mathrm{T}$ helper cell populations in adjacent normal and tumor tissue in relation to the microbiome composition over time would also be needed to determine how these immune and bacterial populations change as a function of time with Anthos treatment.

Overall, the findings from our survey of inflammatory environment changes brought about by Anthos treatment in $\mathrm{Apc}^{\mathrm{Min} /+}$ mice that received ETBF bacteria suggests that Anthos treatment may lead to positive chemopreventive modulation of the inflammatory environment under similar conditions thus reinforcing the benefits of berry-derived compounds on the treatment and prevention of colorectal cancer. 


\section{Abbreviations:}

CRC: colorectal cancer ,IBD: inflammatory bowel disease, Th: T helper cell, TLR: toll-like receptor, IL: interleukin, IFN: interferon, PD-L1: Programmed deathligand 1, PD-1: programmed death 1 receptor, irAEs: immune-related adverse events, T1DM: type 1 diabetes mellitus, SLE: systemic lupus erythematosus, RA: rheumatoid arthritis, CD: crohn's disease, UC: ulcerative colitis, PGE2: prostaglandin E2, EP: Prostaglandin $\mathrm{E}_{2}$ receptor, AhR: aryl hydrocarbon receptor, AhRR: : aryl hydrocarbon receptor repressor, APC: adenomatous polyposis coli, COX-2: cyclo-oxygenase-2, Cy: cyanidin, Dp: delphinidin, Pt: petunidin, Mv: malvidin, Pe: peonidin, HPLC: high performance liquid chromatography, PDA: photodiode array detector, UV: ultraviolet, ETBF: enterotoxigenic Bacteriodes fragilis, BFT: $B$. fragilis toxin, H\&E: Hematoxylin and eosin stain. 
CHAPTER 5:

SUMMARY AND FUTURE DIRECTIONS

\section{Overall goals and specific aims}

The overarching goal of my doctoral work was to determine how Anthos modulates the chemopreventive effects against bacteria- and carcinogeninduced colorectal cancer (CRC). In order to accomplish this task, the specific aims were set forth for the project and are as follows:

Aim 1: Determine anti-proliferative efficacy of optimal mixture of Anthos against colon cancer cells in vitro.

Aim 2: Determine efficacy of Anthos against colorectal cancer using a bacteriadriven $\mathrm{Apc}^{\mathrm{Min} /+}$ ETBF mouse model.

Aim 3: Determine the mechanistic influence of Anthos on ETBF and the environmental carcinogen, $\mathrm{B}[\mathrm{a}] \mathrm{P}$ in $\mathrm{CRC}$.

\section{Major findings from this dissertation}

Although much work has been conducted on the use of plant bioactives as a means of preventing and treating $\mathrm{CRC}$, major limitations in bioavailability, stability and ultimately dosage have prevented its translation to the clinic/patient population. Results from this work show that Anthos treatment leads to the 
ultimate prevention of CRC via several mechanisms including modulation of the phosphorylation state of Src and EGFR and the downstream players in these pathways, modulation of the expression of phase I and detoxifying phase II enzymes and potential harnessing of the gut's ability to protect itself from the invasion of cancer-causing ETBF bacteria/dysbiosis.

\section{Modulation of Src/EGFR}

Prior to this work, it had been shown that APC deficiency was associated with an increase in EGFR activity and c-Src expression in $\mathrm{Apc}^{\mathrm{Min} /+}$ mouse adenomas and intestinal enterocytes [146]. Furthermore, In addition to increasing the secretion of chloride and permeability of intestinal epithelial cells, BFT also activates STAT3 and TH17 responses and leads to increased COX-2 [147]. Additionally, overexpression of COX-2 has been noted in colon tumor tissue and plays a role in the pathogenesis of FAP, ETBF and ultimately colon cancer [148, 149]. However, no work had been conducted to assess the impact of Anthos or anthocyanins on Src in any cancer model or Anthos on EGFR in a CRC model. Results from our look at EGFR, Src, STAT3 and STAT5 phosphorylation status and expression of downstream proteins Cyclin D1, Cyclin D2 and COX-2 showed a significant decrease in Src phosphorylation (Y418), EGFR phosphorylation (Y845) and the downstream mediator STAT5a/b (Y694 and Y699), both in

adjacent normal and colon tumor tissue taken from ETBF treated $\mathrm{Apc}^{\mathrm{Min} /+}$ mice that received Anthos treatment. Furthermore, phosphorylation status of EGFR at Y1068 and Y1173 along with the downstream mediator STAT3 (Y705) was shown to decrease in tumor tissue in Anthos treated mice. Key regulators of cell 
cycle progression and inflammation, including Cyclin D1, Cyclin D2 and COX-2, were shown to be favorably modulated by Anthos, with the most dramatic reductions in expression observed in tumor tissue samples. These highly novel findings provide great insight into how Anthos treatment is leading to such significant decreases in colon tumor numbers in this model.

\section{Phase I/II enzyme balance}

The role of the gut microbiome in the development of $C R C$ has gained attention recently. However, little was previously known about the role of bacteria in modulating the balance between phase I and phase II enzyme expression in the liver and colon. Results from this series of studies showed that the ETBF bacteria led to significantly decreased expression of the phase II enzymes UGT1A6 and GSTM1 in in the liver while increasing the expression of the phase I enzymes CYP1A1 and CYP1B1 in the colon. Anthos and ExoAnthos treatment was also shown to decrease expression of phase I enzymes while increasing phase II enzyme expression in the colon and liver compared to ETBF treated control animals. Additionally, modulation of key transcription factor and regulators including AhR and AhRR, were shown to be induced in ETBF treated animals. Furthermore, Anthos and ExoAnthos treatment appeared to lead to positive changes in AhR, AhRR, ARNT1, and VDR expression in ETBF treated animals. Importantly, no significant differences in expression were noted for phase II enzymes in colon tumor tissue taken from Anthos versus vehicle control animals thereby alleviating potential concerns over increased degradation/excretion of chemo therapeutic drugs. An interesting and highly novel use for this finding 
would be to potentially use Anthos treatment as a way to prevent issues of offsite toxicity for chemotherapeutic drugs such as irinotecan, given the significant increases in phase II enzyme expression that were noted in normal colon tissue gathered from Anthos-treated animals.

In addition to examining the impact of Anthos treatment on the balance of phase I/II enzyme expression using a bacterially driven $\mathrm{Apc}^{\mathrm{Min} /+}$ colon cancer model, we also determined the influence of Anthos treatment on enzyme balance when cells are undergoing insult by the ubiquitous environmental carcinogen, $\mathrm{B}[\mathrm{a}] \mathrm{P}$ in vitro. Results from this series of studies showed that Anthos treatment led to a dosedependent decrease back to baseline in cells treated with the carcinogen. These results combined with the highly novel findings regarding the influence of the ETBF bacteria on the enzyme balance suggest that the combined insult by gut bacterial dysbiosis and environmental carcinogens such as $\mathrm{B}[\mathrm{a}] \mathrm{P}$ could provide the perfect toxic storm and ultimately lead to the development of CRC. Furthermore, Anthos treatment appeared to reverse these alterations both in vitro and in vivo shifting the equilibrium toward a detoxification state.

\section{Inflammation}

Given the significant role that inflammation plays in $C R C$, we sought to determine the influence of Anthos treatment on cytokine and immunoregulatory expression in colon adjacent normal and tumor tissue. Results from this work show novel and highly significant modulation of the immune checkpoint protein, PD-L1 by Anthos in vivo. Modulation of this key protein may be a result of Anthos role in 
decreasing the phosphorylation status of Src and EGFR, which ultimately was shown to alter STAT3 phosphorylation status in in vivo colon tumor tissue, a key regulator of PD-L1 transcription. In vitro work with the human colon cancer cell line, HT-29, confirmed that Anthos treatment led to a dose-dependent decrease in phosphorylation of EGFR (Y1068) and expression of PD-L1. Upregulation of PD-L1 in normal colon tissue may be induced by the increased IFN- $\gamma$ expression that was noted in this tissue. Although a more intensive study of the immune regulatory environment is needed to more conclusively assess the true influence that Anthos has on the colon immune response, the survey presented here provides a highly intriguing look at how Anthos may be working in this bacteriallydriven model of CRC. This combined with the highly significant decreases in colon tumor numbers in Anthos treated animals further confirm this beneficial influence which may be of great use in the clinical setting as both a protective as well as therapeutic treatment.

\section{Influence of Anthos dose}

Given the intriguing results that we gathered from our initial in vivo Anthos and ExoAnthos studies, we carried out a dose-escalation study. The follow-up dose escalation study of Anthos in $\mathrm{Apc}^{\mathrm{Min} /+}$ mice treated with ETBF bacteria showed that there was a clear dose-dependent decrease in the number of colon tumors in mice treated with varying doses of Anthos. At the highest dose of 240 
$\mathrm{mg} / \mathrm{kg} / \mathrm{week}$ which equates to daily dose of about $34 \mathrm{mg} / \mathrm{kg}$ led to greater than $86 \%$ reduction in the formation of colon adenomas.

Although identifying all of the mechanisms by which Anthos work to modulate the development of CRC is beyond the scope of my project, the findings contained within this dissertation make important strides toward identifying the intricate and highly interdependent mechanisms by which this ultimate goal is achieved. From ancient times, plant-derived compounds such as anthocyanins have been key staples both within our diets and as medicinal remedies. Unfortunately, the multifaceted approach by which these compounds work is sometimes lost due to their seemingly mundane origins. With this in mind, the author of this dissertation hopes that one of the major messages that the reader will derive from this work is the strength and complexity that natural compounds such as Anthos exert against diseases such as CRC. Furthermore, the studies presented in this dissertation are merely the first step toward understanding how Anthos work, therefore, additional studies are indeed warranted, thus leading us to the final étape of this dissertation; future directions.

\section{Future Directions}

Future directions for this work would include conducting an in vivo experiment to assess the potential dialogue between $\mathrm{B}[\mathrm{a}] \mathrm{P}$ and dysbiosis of the gut microbiome induced by ETBF bacteria and how Anthos treatment may alter this hypothesized interaction. The results presented in this dissertation suggest that $\mathrm{B}[\mathrm{a}] \mathrm{P}$ and ETBF appear to shift the phase $\mathrm{I} / \mathrm{I}$ enzyme equilibrium in a similar manner and 
thus may have a detrimental synergistic activity that could promote the formation of the active carcinogen BPDE. Additional work assessing the impact of Anthos treatment in non-transformed colon cells treated with $\mathrm{B}[\mathrm{a}] \mathrm{P}$ along with the formation of BPDE-DNA adducts would also be the logical next steps for this work. Ultimately, research into this area is made especially pressing given the recent rise in $\mathrm{CRC}$ in younger individuals.

Additional in vitro work to assess the role of Anthos in modulating $\mathrm{T}$ helper cell balance would also be an important future project to undertake. Linking PD-L1 and the known side effect of colitis with our findings in our in vivo ETBF Apc $\mathrm{Min} /+^{-}$ mouse model treated with Anthos could be a key finding in overcoming the current limitations of this breakthrough immunotherapy treatment. The ultimate goal of this research is clinical translation of these findings. Therefore, conducting a clinical trial with these compounds is also planned by our laboratory for the future given the highly promising in vitro and in vivo results presented in this dissertation. 


\section{REFERENCES}

[1] M. Arnold, M.S. Sierra, M. Laversanne, I. Soerjomataram, A. Jemal, F. Bray, Global patterns and trends in colorectal cancer incidence and mortality, Gut, 66 (2017) 683.

[2] G. Murphy, S.S. Devesa, A.J. Cross, P.D. Inskip, K.A. McGlynn, M.B. Cook, Sex Disparities in Colorectal Cancer Incidence by Anatomic Subsite, Race and Age, International journal of cancer. Journal international du cancer, 128 (2011) 1668-1675.

[3] N. Shivappa, A. Zucchetto, M. Montella, D. Serraino, S.E. Steck, C. La Vecchia, J.R. Hebert, Inflammatory potential of diet and risk of colorectal cancer: a case-control study from Italy, The British journal of nutrition, 114 (2015) 152158.

[4] A. Jemal, F. Bray, M.M. Center, J. Ferlay, E. Ward, D. Forman, Global cancer statistics, CA: a cancer journal for clinicians, 61 (2011) 69-90.

[5] S. Pathy, R. Lambert, C. Sauvaget, R. Sankaranarayanan, The incidence and survival rates of colorectal cancer in India remain low compared with rising rates in East Asia, Diseases of the colon and rectum, 55 (2012) 900-906.

[6] A.B. Kunnumakkara, S. Guha, B.B. Aggarwal, Curcumin and colorectal cancer: Add spice to your life, Current Colorectal Cancer Reports, 5 (2009) 5.

[7] O. Manousos, N.E. Day, D. Trichopoulos, F. Gerovassilis, A. Tzonou, A. Polychronopoulou, Diet and colorectal cancer: A case-control study in Greece, International journal of cancer, 32 (1983) 1-5.

[8] P. Terry, E. Giovannucci, K.B. Michels, L. Bergkvist, H. Hansen, L. Holmberg, A. Wolk, Fruit, Vegetables, Dietary Fiber, and Risk of Colorectal Cancer, JNCl: Journal of the National Cancer Institute, 93 (2001) 525-533.

[9] J. Lee, A. Shin, J.H. Oh, J. Kim, Colors of vegetables and fruits and the risks of colorectal cancer, World Journal of Gastroenterology, 23 (2017) 2527-2538.

[10] E. Vogtmann, Y.-B. Xiang, H.-L. Li, E.B. Levitan, G. Yang, J.W. Waterbor, J. Gao, H. Cai, L. Xie, Q.-J. Wu, B. Zhang, Y.-T. Gao, W. Zheng, X.-O. Shu, Fruit and vegetable intake and the risk of colorectal cancer: Results from the Shanghai Men's Health Study, Cancer causes \& control : CCC, 24 (2013) 1935-1945. 
[11] H. Wu, Q. Dai, M.J. Shrubsole, R.M. Ness, D. Schlundt, W.E. Smalley, H. Chen, M. Li, Y. Shyr, W. Zheng, Fruit and vegetable intakes are associated with lower risk of colorectal adenomas, The Journal of nutrition, 139 (2009) 340-344.

[12] R.L. Siegel, S.A. Fedewa, W.F. Anderson, K.D. Miller, J. Ma, P.S. Rosenberg, A. Jemal, Colorectal Cancer Incidence Patterns in the United States, 1974-2013, Journal of the National Cancer Institute, 109 (2017).

[13] J. Terzić, S. Grivennikov, E. Karin, M. Karin, Inflammation and Colon Cancer, Gastroenterology, 138 (2010) 2101-2114.e2105.

[14] S.L. Schmit, H.S. Rennert, G. Rennert, S.B. Gruber, Coffee Consumption and the Risk of Colorectal Cancer, Cancer epidemiology, biomarkers \& prevention : a publication of the American Association for Cancer Research, cosponsored by the American Society of Preventive Oncology, 25 (2016) 634639.

[15] G. Yang, W. Zheng, Y.B. Xiang, J. Gao, H.L. Li, X. Zhang, Y.T. Gao, X.O. Shu, Green tea consumption and colorectal cancer risk: a report from the Shanghai Men's Health Study, Carcinogenesis, 32 (2011) 1684-1688.

[16] J.N. Poynter , S.B. Gruber , P.D.R. Higgins, R. Almog , J.D. Bonner , H.S. Rennert, M. Low , J.K. Greenson, G. Rennert Statins and the Risk of Colorectal Cancer, New England Journal of Medicine, 352 (2005) 2184-2192.

[17] L.A. Garcia-Rodriguez, C. Huerta-Alvarez, Reduced risk of colorectal cancer among long-term users of aspirin and nonaspirin nonsteroidal antiinflammatory drugs, Epidemiology (Cambridge, Mass.), 12 (2001) 88-93.

[18] M. Howsam, J.O. Grimalt, E. Guinó, M. Navarro, J. Martí-Ragué, M.A. Peinado, G. Capellá, V. Moreno, G. for the Bellvitge Colorectal Cancer, Organochlorine Exposure and Colorectal Cancer Risk, Environmental Health Perspectives, 112 (2004) 1460-1466.

[19] N. Kazerouni, R. Sinha, C.-H. Hsu, A. Greenberg, N. Rothman, Analysis of 200 food items for benzo[a]pyrene and estimation of its intake in an epidemiologic study, Food and Chemical Toxicology, 39 (2001) 423-436.

[20] A.M. El-Tawil, Colorectal cancer and pollution, World Journal of Gastroenterology : WJG, 16 (2010) 3475-3477.

[21] L. Rushton, HOW MUCH DOES THE ENVIRONMENT CONTRIBUTE TO CANCER?, Occupational and Environmental Medicine, 60 (2003) 150. 
[22] E. Oddone, C. Modonesi, G. Gatta, Occupational exposures and colorectal cancers: A quantitative overview of epidemiological evidence, World Journal of Gastroenterology : WJG, 20 (2014) 12431-12444.

[23] G.P. Pfeifer, M.F. Denissenko, M. Olivier, N. Tretyakova, S.S. Hecht, P. Hainaut, Tobacco smoke carcinogens, DNA damage and p53 mutations in smoking-associated cancers, Oncogene, 21 (2002) 7435-7451.

[24] C.L. Sears, D.M. Pardoll, Perspective: Alpha-Bugs, Their Microbial Partners, and the Link to Colon Cancer, The Journal of Infectious Diseases, 203 (2011) 306-311.

[25] S. Wu, K.C. Lim, J. Huang, R.F. Saidi, C.L. Sears, Bacteroides fragilis enterotoxin cleaves the zonula adherens protein, E-cadherin, Proceedings of the National Academy of Sciences of the United States of America, 95 (1998) 1497914984.

[26] S. Wu, P.J. Morin, D. Maouyo, C.L. Sears, Bacteroides fragilis enterotoxin induces c-Myc expression and cellular proliferation, Gastroenterology, 124 (2003) 392-400.

[27] F. Housseau, C.L. Sears, Enterotoxigenic Bacteroides fragilis (ETBF)mediated colitis in Min (Apc+/-) mice: a human commensal-based murine model of colon carcinogenesis, Cell cycle (Georgetown, Tex.), 9 (2010) 3-5.

[28] C.M. Dejea, P. Fathi, J.M. Craig, A. Boleij, R. Taddese, A.L. Geis, X. Wu, C.E. DeStefano Shields, E.M. Hechenbleikner, D.L. Huso, R.A. Anders, F.M. Giardiello, E.C. Wick, H. Wang, S. Wu, D.M. Pardoll, F. Housseau, C.L. Sears, Patients with familial adenomatous polyposis harbor colonic biofilms containing tumorigenic bacteria, Science (New York, N.Y.), 359 (2018) 592-597.

[29] Y. Imamura, T. Morikawa, X. Liao, P. Lochhead, A. Kuchiba, M. Yamauchi, Z.R. Qian, R. Nishihara, J.A. Meyerhardt, K.M. Haigis, C.S. Fuchs, S. Ogino, Specific mutations in KRAS codons 12 and 13, and patient prognosis in 1075 BRAF wild-type colorectal cancers, Clinical cancer research : an official journal of the American Association for Cancer Research, 18 (2012) 4753-4763.

[30] W. Li, T. Qiu, Y. Ling, L. Guo, L. Li, J. Ying, Molecular pathological epidemiology of colorectal cancer in Chinese patients with KRAS and BRAF mutations, Oncotarget, 6 (2015) 39607-39613.

[31] C.E. Atreya, Z. Sangale, N. Xu, M.R. Matli, E. Tikishvili, W. Welbourn, S. Stone, K.M. Shokat, R.S. Warren, PTEN expression is consistent in colorectal cancer primaries and metastases and associates with patient survival, Cancer medicine, 2 (2013) 496-506. 
[32] Y. Chen, Y. Shi, J. Lin, Y.B. Ye, X.J. Wang, G. Chen, Z.Q. Guo, Combined Analysis of EGFR and PTEN Status in Patients With KRAS Wild-Type Metastatic Colorectal Cancer, Medicine, 94 (2015) e1698.

[33] M. Han, G. Wu, P. Sun, J. Nie, J. Zhang, Y. Li, Association of genetic polymorphisms in PTEN and additional interaction with alcohol consumption and smoking on colorectal cancer in Chinese population, International Journal of Clinical and Experimental Medicine, 8 (2015) 21629-21634.

[34] J.L. Boulay, G. Mild, A. Lowy, J. Reuter, M. Lagrange, L. Terracciano, U. Laffer, R. Herrmann, C. Rochlitz, SMAD7 is a prognostic marker in patients with colorectal cancer, International journal of cancer, 104 (2003) 446-449.

[35] A.J. Levine, A.I. Phipps, J.A. Baron, D.D. Buchanan, D.J. Ahnen, S.A. Cohen, N.M. Lindor, P.A. Newcomb, C. Rosty, R.W. Haile, P.W. Laird, D.J. Weisenberger, Clinicopathologic Risk Factor Distributions for MLH1 Promoter Region Methylation in CIMP-Positive Tumors, Cancer epidemiology, biomarkers \& prevention : a publication of the American Association for Cancer Research, cosponsored by the American Society of Preventive Oncology, 25 (2016) 68-75.

[36] S.P. Cleary, M. Cotterchio, M.A. Jenkins, H. Kim, R. Bristow, R. Green, R. Haile, J.L. Hopper, L. LeMarchand, N. Lindor, P. Parfrey, J. Potter, B. Younghusband, S. Gallinger, Germline MutY human homologue mutations and colorectal cancer: a multisite case-control study, Gastroenterology, 136 (2009) 1251-1260.

[37] M. Lüchtenborg, M.P. Weijenberg, G.M.J.M. Roemen, A.P. de Bruïne, P.A. van den Brandt, M.H.F.M. Lentjes, M. Brink, M. van Engeland, R.A. Goldbohm, A.F.P.M. de Goeij, APC mutations in sporadic colorectal carcinomas from The Netherlands Cohort Study, Carcinogenesis, 25 (2004) 1219-1226.

[38] M.A. Hildebrandt, M.E. Reyes, M. Lin, Y. He, S.V. Nguyen, E.T. Hawk, X. Wu, Germline Genetic Variants in the Wnt/beta-Catenin Pathway as Predictors of Colorectal Cancer Risk, Cancer epidemiology, biomarkers \& prevention : a publication of the American Association for Cancer Research, cosponsored by the American Society of Preventive Oncology, 25 (2016) 540-546.

[39] R.N. Jorissen, M. Christie, D. Mouradov, A. Sakthianandeswaren, S. Li, C. Love, Z.Z. Xu, P.L. Molloy, I.T. Jones, S. McLaughlin, R.L. Ward, N.J. Hawkins, A.R. Ruszkiewicz, J. Moore, A.W. Burgess, D. Busam, Q. Zhao, R.L. Strausberg, L. Lipton, J. Desai, P. Gibbs, O.M. Sieber, Wild-type APC predicts poor prognosis in microsatellite-stable proximal colon cancer, British journal of cancer, 113 (2015) 979-988. 
[40] W.S. Samowitz, M.L. Slattery, C. Sweeney, J. Herrick, R.K. Wolff, H. Albertsen, APC mutations and other genetic and epigenetic changes in colon cancer, Molecular cancer research : MCR, 5 (2007) 165-170.

[41] M.S. Pino, D.C. Chung, The chromosomal instability pathway in colon cancer, Gastroenterology, 138 (2010) 2059-2072.

[42] E.R. Fearon, B. Vogelstein, A genetic model for colorectal tumorigenesis, Cell, 61 (1990) 759-767.

[43] S. Markowitz, J. Wang, L. Myeroff, R. Parsons, L. Sun, J. Lutterbaugh, R.S. Fan, E. Zborowska, K.W. Kinzler, B. Vogelstein, et al., Inactivation of the type II TGF-beta receptor in colon cancer cells with microsatellite instability, Science (New York, N.Y.), 268 (1995) 1336-1338.

[44] Y. Samuels, V.E. Velculescu, Oncogenic mutations of PIK3CA in human cancers, Cell cycle (Georgetown, Tex.), 3 (2004) 1221-1224.

[45] S.J. Baker, E.R. Fearon, J.M. Nigro, S.R. Hamilton, A.C. Preisinger, J.M. Jessup, P. vanTuinen, D.H. Ledbetter, D.F. Barker, Y. Nakamura, R. White, B. Vogelstein, Chromosome 17 deletions and p53 gene mutations in colorectal carcinomas, Science (New York, N.Y.), 244 (1989) 217-221.

[46] K.M. Haigis, K.R. Kendall, Y. Wang, A. Cheung, M.C. Haigis, J.N. Glickman, M. Niwa-Kawakita, A. Sweet-Cordero, J. Sebolt-Leopold, K.M. Shannon, J. Settleman, M. Giovannini, T. Jacks, Differential effects of oncogenic K-Ras and $\mathrm{N}$-Ras on proliferation, differentiation and tumor progression in the colon, Nature genetics, 40 (2008) 600-608.

[47] J.N. Poynter, K.D. Siegmund, D.J. Weisenberger, T.I. Long, S.N. Thibodeau, N. Lindor, J. Young, M.A. Jenkins, J.L. Hopper, J.A. Baron, D. Buchanan, G. Casey, A.J. Levine, L.L. Marchand, S. Gallinger, B. Bapat, J.D. Potter, P.A. Newcomb, R.W. Haile, t.C.C.F.R.I. Laird, W. Peter, Molecular characterization of $\mathrm{MSI}-\mathrm{H}$ colorectal cancer by MLHI promoter methylation, immunohistochemistry and mismatch repair germline mutation screening, Cancer epidemiology, biomarkers \& prevention : a publication of the American Association for Cancer Research, cosponsored by the American Society of Preventive Oncology, 17 (2008) 3208-3215.

[48] C.R. Boland, A. Goel, Microsatellite Instability in Colorectal Cancer, Gastroenterology, 138 (2010) 2073-2087.e2073.

[49] C.C. Pritchard, W.M. Grady, Colorectal cancer molecular biology moves into clinical practice, Gut, (2010). 
[50] C.H. Kim, J.W. Huh, H.R. Kim, Y.J. Kim, CpG island methylator phenotype is an independent predictor of survival after curative resection for colorectal cancer: A prospective cohort study, J Gastroenterol Hepatol, (2017).

[51] D.L. Worthley, B.A. Leggett, Colorectal cancer: molecular features and clinical opportunities, The Clinical biochemist. Reviews / Australian Association of Clinical Biochemists, 31 (2010) 31-38.

[52] S.D. Markowitz, M.M. Bertagnolli, Molecular origins of cancer: Molecular basis of colorectal cancer, The New England journal of medicine, 361 (2009) 2449-2460.

[53] S. Ogino, A. Goel, Molecular classification and correlates in colorectal cancer, The Journal of molecular diagnostics : JMD, 10 (2008) 13-27.

[54] J.R. Jass, Classification of colorectal cancer based on correlation of clinical, morphological and molecular features, Histopathology, 50 (2007) 113-130.

[55] C.G. Moertel, T.R. Fleming, J.S. Macdonald, D.G. Haller, J.A. Laurie, P.J. Goodman, J.S. Ungerleider, W.A. Emerson, D.C. Tormey, J.H. Glick, et al., Levamisole and fluorouracil for adjuvant therapy of resected colon carcinoma, The New England journal of medicine, 322 (1990) 352-358.

[56] J.A. Martenson, G. Hyland, C.G. Moertel, J.A. Mailliard, J.R. O'Fallon, R.T. Collins, R.F. Morton, H.H. Tewfik, R.L. Moore, A.R. Frank, R.E. Urias, R.L. Deming, Olsalazine is contraindicated during pelvic radiation therapy: Results of a double-blind, randomized clinical trial, International Journal of Radiation Oncology*Biology ${ }^{\star}$ Physics, 35 (1996) 299-303.

[57] C.G. Moertel, T.R. Fleming, J.S. Macdonald, D.G. Haller, J.A. Laurie, Hepatic toxicity associated with fluorouracil plus levamisole adjuvant therapy, Journal of Clinical Oncology, 11 (1993) 2386-2390.

[58] A. Moriarity, J. O'Sullivan, J. Kennedy, B. Mehigan, P. McCormick, Current targeted therapies in the treatment of advanced colorectal cancer: a review, Therapeutic Advances in Medical Oncology, 8 (2016) 276-293.

[59] A. Prat, E. Casado, J. Cortes, New approaches in angiogenic targeting for colorectal cancer, World J Gastroenterol, 13 (2007) 5857-5866.

[60] H. Linardou, H. Gogas, Toxicity management of immunotherapy for patients with metastatic melanoma, Annals of Translational Medicine, 4 (2016) 272.

[61] S. De Dosso, C. Sessa, P. Saletti, Adjuvant therapy for colon cancer: present and perspectives, Cancer treatment reviews, 35 (2009) 160-166. 
[62] G. Recondo, Jr., E. Díaz-Cantón, M. de la Vega, M. Greco, G. Recondo, Sr., M.E. Valsecchi, Advances and new perspectives in the treatment of metastatic colon cancer, World Journal of Gastrointestinal Oncology, 6 (2014) 211-224.

[63] L. Migliore, F. Migheli, R. Spisni, F. Coppede, Genetics, cytogenetics, and epigenetics of colorectal cancer, Journal of biomedicine \& biotechnology, 2011 (2011) 792362.

[64] P. Gervaz, P. Bucher, P. Morel, Two colons-two cancers: paradigm shift and clinical implications, Journal of surgical oncology, 88 (2004) 261-266.

[65] E. Half, D. Bercovich, P. Rozen, Familial adenomatous polyposis, Orphanet journal of rare diseases, 4 (2009) 22.

[66] K.W. Jasperson, T.M. Tuohy, D.W. Neklason, R.W. Burt, Hereditary and familial colon cancer, Gastroenterology, 138 (2010) 2044-2058.

[67] P. Galiatsatos, W.D. Foulkes, Familial Adenomatous Polyposis, Am J Gastroenterol, 101 (2006) 385-398.

[68] P. Polakis, The adenomatous polyposis coli (APC) tumor suppressor, Biochimica et Biophysica Acta (BBA) - Reviews on Cancer, 1332 (1997) F127F147.

[69] K. Aoki, M.M. Taketo, Adenomatous polyposis coli (APC): a multi-functional tumor suppressor gene, Journal of cell science, 120 (2007) 3327-3335.

[70] A. Sameer, Colorectal Cancer: Molecular Mutations and Polymorphisms, Frontiers in Oncology, 3 (2013).

[71] M.L. Bisgaard, K. Fenger, S. Bulow, E. Niebuhr, J. Mohr, Familial adenomatous polyposis (FAP): frequency, penetrance, and mutation rate, Human mutation, 3 (1994) 121-125.

[72] M.L. Falcone Ferreyra, S.P. Rius, P. Casati, Flavonoids: biosynthesis, biological functions, and biotechnological applications, Frontiers in Plant Science, 3 (2012) 222.

[73] J.B. Harborne, C.A. Williams, Advances in flavonoid research since 1992, Phytochemistry, 55 (2000) 481-504.

[74] B. Yousuf, K. Gul, A.A. Wani, P. Singh, Health Benefits of Anthocyanins and Their Encapsulation for Potential Use in Food Systems: A Review, Crit Rev Food Sci, 56 (2016) 2223-2230.

[75] C.R. Welch, Q. Wu, J.E. Simon, Recent Advances in Anthocyanin Analysis and Characterization, Current analytical chemistry, 4 (2008) 75-101. 
[76] R.L. Prior, X. Wu, Anthocyanins: Structural characteristics that result in unique metabolic patterns and biological activities, Free Radical Research, 40 (2006) 1014-1028.

[77] M. Landi, M. Tattini, K.S. Gould, Multiple functional roles of anthocyanins in plant-environment interactions, Environmental and Experimental Botany, 119 (2015) 4-17.

[78] H. Kausar, J. Jeyabalan, F. Aqil, D. Chabba, J. Sidana, I.P. Singh, R.C. Gupta, Berry anthocyanidins synergistically suppress growth and invasive potential of human non-small-cell lung cancer cells, Cancer Lett, 325 (2012) 5462.

[79] D. Del Rio, G. Borges, A. Crozier, Berry flavonoids and phenolics: bioavailability and evidence of protective effects, The British journal of nutrition, 104 Suppl 3 (2010) S67-90.

[80] K.S. Gould, C. Lister, Flavonoid Functions in Plants, in: O.M. Andersen, K.R. Markham (Eds.) FLAVONOIDS Chemistry, Biochemistry and Applications

CRC Press, Boca Raton, 2006, pp. 397-442.

[81] A. Scalbert, G. Williamson, Dietary intake and bioavailability of polyphenols, The Journal of nutrition, 130 (2000) 2073s-2085s.

[82] J.M. Gee, M.S. DuPont, M.J. Rhodes, I.T. Johnson, Quercetin glucosides interact with the intestinal glucose transport pathway, Free radical biology \& medicine, 25 (1998) 19-25.

[83] T. Miyazawa, K. Nakagawa, M. Kudo, K. Muraishi, K. Someya, Direct intestinal absorption of red fruit anthocyanins, cyanidin-3-glucoside and cyanidin3,5-diglucoside, into rats and humans, J Agric Food Chem, 47 (1999) 1083-1091.

[84] S. Talavéra, C. Felgines, O. Texier, C. Besson, A. Gil-Izquierdo, J.-L. Lamaison, C. Rémésy, Anthocyanin Metabolism in Rats and Their Distribution to Digestive Area, Kidney, and Brain, Journal of Agricultural and Food Chemistry, 53 (2005) 3902-3908.

[85] A.R. Rechner, C. Kroner, Anthocyanins and colonic metabolites of dietary polyphenols inhibit platelet function, Thrombosis Research, 116 (2005) 327-334.

[86] N. Katsube, K. Iwashita, T. Tsushida, K. Yamaki, M. Kobori, Induction of apoptosis in cancer cells by Bilberry (Vaccinium myrtillus) and the anthocyanins, J Agric Food Chem, 51 (2003) 68-75.

[87] C. Zhao, M.M. Giusti, M. Malik, M.P. Moyer, B.A. Magnuson, Effects of commercial anthocyanin-rich extracts on colonic cancer and nontumorigenic colonic cell growth, J Agric Food Chem, 52 (2004) 6122-6128. 
[88] D.Y. Shin, W.S. Lee, J.N. Lu, M.H. Kang, C.H. Ryu, G.Y. Kim, H.S. Kang, S.C. Shin, Y.H. Choi, Induction of apoptosis in human colon cancer HCT-116 cells by anthocyanins through suppression of Akt and activation of p38-MAPK, International journal of oncology, 35 (2009) 1499-1504.

[89] N. Karthi, T. Kalaiyarasu, S. Kandakumar, P. Mariyappan, V. Manju, Pelargonidin induces apoptosis and cell cycle arrest via a mitochondria mediated intrinsic apoptotic pathway in HT29 cells, RSC Advances, 6 (2016) 45064-45076.

[90] J.-M. Yun, F. Afaq, N. Khan, H. Mukhtar, Delphinidin, an Anthocyanidin in Pigmented Fruits and Vegetables, Induces Apoptosis and Cell Cycle Arrest in Human Colon Cancer HCT116 Cells, Molecular carcinogenesis, 48 (2009) 260270.

[91] M.R. Webb, K. Min, S.E. Ebeler, Anthocyanin Interactions with DNA: Intercalation, Topoisomerase I Inhibition and Oxidative Reactions, Journal of food biochemistry, 32 (2008) 576-596.

[92] M.Y. Park, J.M. Kim, J.S. Kim, M.G. Choung, M.K. Sung, Chemopreventive Action of Anthocyanin-rich Black Soybean Fraction in APC (Min/+) Intestinal Polyposis Model, Journal of cancer prevention, 20 (2015) 193-201.

[93] H. Cai, T.H. Marczylo, N. Teller, K. Brown, W.P. Steward, D. Marko, A.J. Gescher, Anthocyanin-rich red grape extract impedes adenoma development in the Apc(Min) mouse: pharmacodynamic changes and anthocyanin levels in the murine biophase, European journal of cancer (Oxford, England : 1990), 46 (2010) 811-817.

[94] L. Li, L. Wang, Z. Wu, L. Yao, Y. Wu, L. Huang, K. Liu, X. Zhou, D. Gou, Anthocyanin-rich fractions from red raspberries attenuate inflammation in both RAW264.7 macrophages and a mouse model of colitis, Scientific reports, 4 (2014) 6234.

[95] D. Cooke, M. Schwarz, D. Boocock, P. Winterhalter, W.P. Steward, A.J. Gescher, T.H. Marczylo, Effect of cyanidin-3-glucoside and an anthocyanin mixture from bilberry on adenoma development in the ApcMin mouse model of intestinal carcinogenesis--relationship with tissue anthocyanin levels, International journal of cancer, 119 (2006) 2213-2220.

[96] E.J. Freireich, E.A. Gehan, D.P. Rall, L.H. Schmidt, H.E. Skipper, Quantitative comparison of toxicity of anticancer agents in mouse, rat, hamster, dog, monkey, and man, Cancer chemotherapy reports, 50 (1966) 219-244.

[97] S. Thomasset, D.P. Berry, H. Cai, K. West, T.H. Marczylo, D. Marsden, K. Brown, A. Dennison, G. Garcea, A. Miller, D. Hemingway, W.P. Steward, A.J. 
Gescher, Pilot study of oral anthocyanins for colorectal cancer chemoprevention, Cancer prevention research (Philadelphia, Pa.), 2 (2009) 625-633.

[98] R.A. Mentor-Marcel, G. Bobe, C. Sardo, L.S. Wang, C.T. Kuo, G. Stoner, N.H. Colburn, Plasma cytokines as potential response indicators to dietary freeze-dried black raspberries in colorectal cancer patients, Nutrition and cancer, 64 (2012) 820-825.

[99] L.S. Wang, M. Arnold, Y.W. Huang, C. Sardo, C. Seguin, E. Martin, T.H. Huang, K. Riedl, S. Schwartz, W. Frankel, D. Pearl, Y. Xu, J. Winston, 3rd, G.Y. Yang, G. Stoner, Modulation of genetic and epigenetic biomarkers of colorectal cancer in humans by black raspberries: a phase I pilot study, Clinical cancer research : an official journal of the American Association for Cancer Research, 17 (2011) 598-610.

[100] S. Roth, M.R. Spalinger, C. Gottier, L. Biedermann, J. Zeitz, S. Lang, A. Weber, G. Rogler, M. Scharl, Bilberry-Derived Anthocyanins Modulate Cytokine Expression in the Intestine of Patients with Ulcerative Colitis, PloS one, 11 (2016) e0154817.

[101] L. Biedermann, J. Mwinyi, M. Scharl, P. Frei, J. Zeitz, G.A. Kullak-Ublick, S.R. Vavricka, M. Fried, A. Weber, H.U. Humpf, S. Peschke, A. Jetter, G. Krammer, G. Rogler, Bilberry ingestion improves disease activity in mild to moderate ulcerative colitis - an open pilot study, Journal of Crohn's \& colitis, 7 (2013) 271-279.

[102] S. Kamiloglu, E. Capanoglu, C. Grootaert, J. Van Camp, Anthocyanin Absorption and Metabolism by Human Intestinal Caco-2 Cells--A Review, International journal of molecular sciences, 16 (2015) 21555-21574.

[103] C. Thery, L. Zitvogel, S. Amigorena, Exosomes: composition, biogenesis and function, Nat Rev Immunol, 2 (2002) 569-579.

[104] R.M. Johnstone, A. Bianchini, K. Teng, Reticulocyte maturation and exosome release: transferrin receptor containing exosomes shows multiple plasma membrane functions, Blood, 74 (1989) 1844-1851.

[105] S.R. Vaiselbuh, Exosomes in Cancer Research, Cancer Research Frontiers, 1 (2015) 11-24.

[106] J. Zhang, S. Li, L. Li, M. Li, C. Guo, J. Yao, S. Mi, Exosome and Exosomal MicroRNA: Trafficking, Sorting, and Function, Genomics, Proteomics \& Bioinformatics, 13 (2015) 17-24.

[107] J. Kowal, M. Tkach, C. Thery, Biogenesis and secretion of exosomes, Current opinion in cell biology, 29 (2014) 116-125. 
[108] G. Raposo, W. Stoorvogel, Extracellular vesicles: exosomes, microvesicles, and friends, The Journal of cell biology, 200 (2013) 373-383.

[109] C. Théry, M. Boussac, P. Véron, P. Ricciardi-Castagnoli, G. Raposo, J. Garin, S. Amigorena, Proteomic Analysis of Dendritic Cell-Derived Exosomes: A Secreted Subcellular Compartment Distinct from Apoptotic Vesicles, The Journal of Immunology, 166 (2001) 7309-7318.

[110] B.K. Thakur, H. Zhang, A. Becker, I. Matei, Y. Huang, B. Costa-Silva, Y. Zheng, A. Hoshino, H. Brazier, J. Xiang, C. Williams, R. Rodriguez-Barrueco, J.M. Silva, W. Zhang, S. Hearn, O. Elemento, N. Paknejad, K. ManovaTodorova, K. Welte, J. Bromberg, H. Peinado, D. Lyden, Double-stranded DNA in exosomes: a novel biomarker in cancer detection, Cell Res, 24 (2014) 766769.

[111] H. Valadi, K. Ekstrom, A. Bossios, M. Sjostrand, J.J. Lee, J.O. Lotvall, Exosome-mediated transfer of mRNAs and microRNAs is a novel mechanism of genetic exchange between cells, Nature cell biology, 9 (2007) 654-659.

[112] A. Clayton, J.P. Mitchell, J. Court, M.D. Mason, Z. Tabi, Human tumorderived exosomes selectively impair lymphocyte responses to interleukin-2, Cancer research, 67 (2007) 7458-7466.

[113] C. Thery, Exosomes: secreted vesicles and intercellular communications, F1000 biology reports, 3 (2011) 15.

[114] I. Parolini, C. Federici, C. Raggi, L. Lugini, S. Palleschi, A. De Milito, C. Coscia, E. lessi, M. Logozzi, A. Molinari, M. Colone, M. Tatti, M. Sargiacomo, S. Fais, Microenvironmental $\mathrm{pH}$ is a key factor for exosome traffic in tumor cells, The Journal of biological chemistry, 284 (2009) 34211-34222.

[115] R. Munagala, F. Aqil, J. Jeyabalan, R.C. Gupta, Bovine milk-derived exosomes for drug delivery, Cancer Lett, 371 (2016) 48-61.

[116] F. Aqil, H. Kausar, A.K. Agrawal, J. Jeyabalan, A.H. Kyakulaga, R. Munagala, R. Gupta, Exosomal formulation enhances therapeutic response of celastrol against lung cancer, Experimental and molecular pathology, 101 (2016) $12-21$.

[117] A.K. Agrawal, F. Aqil, J. Jeyabalan, W.A. Spencer, J. Beck, B.W. Gachuki, S.S. Alhakeem, K. Oben, R. Munagala, S. Bondada, R.C. Gupta, Milk-derived exosomes for oral delivery of paclitaxel, Nanomedicine : nanotechnology, biology, and medicine, (2017).

[118] V.N. Tudyka, S.K. Clark, Surgical treatment in familial adenomatous polyposis, Annals of Gastroenterology, 25 (2012) 201-206. 
[119] J. Burn, A.-M. Gerdes, F. Macrae, J.-P. Mecklin, G. Moeslein, S. Olschwang, D. Eccles, D.G. Evans, E.R. Maher, L. Bertario, M.-L. Bisgaard, M.G. Dunlop, J.W.C. Ho, S.V. Hodgson, A. Lindblom, J. Lubinski, P.J. Morrison, V. Murday, R. Ramesar, L. Side, R.J. Scott, H.J.W. Thomas, H.F. Vasen, G. Barker, G. Crawford, F. Elliott, M. Movahedi, K. Pylvanainen, J.T. Wijnen, R. Fodde, H.T. Lynch, J.C. Mathers, D.T. Bishop, Long-term effect of aspirin on cancer risk in carriers of hereditary colorectal cancer: an analysis from the CAPP2 randomised controlled trial, The Lancet, 378 (2011) 2081-2087.

[120] C. Carroll, K. Cooper, D. Papaioannou, D. Hind, H. Pilgrim, P. Tappenden, Supplemental calcium in the chemoprevention of colorectal cancer: a systematic review and meta-analysis, Clinical therapeutics, 32 (2010) 789-803.

[121] R.M. Bostick, Effects of supplemental vitamin D and calcium on normal colon tissue and circulating biomarkers of risk for colorectal neoplasms, The Journal of steroid biochemistry and molecular biology, 148 (2015) 86-95.

[122] T. Qin, M. Du, H. Du, Y. Shu, M. Wang, L. Zhu, Folic acid supplements and colorectal cancer risk: meta-analysis of randomized controlled trials, Scientific reports, 5 (2015) 12044.

[123] K.R. Patel, V.A. Brown, D.J. Jones, R.G. Britton, D. Hemingway, A.S. Miller, K.P. West, T.D. Booth, M. Perloff, J.A. Crowell, D.E. Brenner, W.P. Steward, A.J. Gescher, K. Brown, Clinical pharmacology of resveratrol and its metabolites in colorectal cancer patients, Cancer research, 70 (2010) 7392-7399.

[124] R.A. Sharma, H.R. McLelland, K.A. Hill, C.R. Ireson, S.A. Euden, M.M. Manson, M. Pirmohamed, L.J. Marnett, A.J. Gescher, W.P. Steward, Pharmacodynamic and pharmacokinetic study of oral Curcuma extract in patients with colorectal cancer, Clinical cancer research : an official journal of the American Association for Cancer Research, 7 (2001) 1894-1900.

[125] M. Shimizu, Y. Fukutomi, M. Ninomiya, K. Nagura, T. Kato, H. Araki, M. Suganuma, H. Fujiki, H. Moriwaki, Green tea extracts for the prevention of metachronous colorectal adenomas: a pilot study, Cancer epidemiology, biomarkers \& prevention : a publication of the American Association for Cancer Research, cosponsored by the American Society of Preventive Oncology, 17 (2008) 3020-3025.

[126] K.F. Adams, P.D. Lampe, K.M. Newton, J.T. Ylvisaker, A. Feld, D. Myerson, S.S. Emerson, E. White, J.D. Potter, J.W. Lampe, Soy protein containing isoflavones does not decrease colorectal epithelial cell proliferation in a randomized controlled trial, The American journal of clinical nutrition, 82 (2005) 620-626. 
[127] M.A. Nunez-Sanchez, R. Garcia-Villalba, T. Monedero-Saiz, N.V. GarciaTalavera, M.B. Gomez-Sanchez, C. Sanchez-Alvarez, A.M. Garcia-Albert, F.J. Rodriguez-Gil, M. Ruiz-Marin, F.A. Pastor-Quirante, F. Martinez-Diaz, M.J. Yanez-Gascon, A. Gonzalez-Sarrias, F.A. Tomas-Barberan, J.C. Espin, Targeted metabolic profiling of pomegranate polyphenols and urolithins in plasma, urine and colon tissues from colorectal cancer patients, Molecular nutrition \& food research, 58 (2014) 1199-1211.

[128] K. Cooper, H. Squires, C. Carroll, D. Papaioannou, A. Booth, R.F. Logan, C. Maguire, D. Hind, P. Tappenden, Chemoprevention of colorectal cancer: systematic review and economic evaluation, Health technology assessment (Winchester, England), 14 (2010) 1-206.

[129] M.A. Nunez-Sanchez, A. Gonzalez-Sarrias, M. Romo-Vaquero, R. GarciaVillalba, M.V. Selma, F.A. Tomas-Barberan, M.T. Garcia-Conesa, J.C. Espin, Dietary phenolics against colorectal cancer--From promising preclinical results to poor translation into clinical trials: Pitfalls and future needs, Molecular nutrition \& food research, 59 (2015) 1274-1291.

[130] J. Jeyabalan, F. Aqil, R. Munagala, L. Annamalai, M.V. Vadhanam, R.C. Gupta, Chemopreventive and therapeutic activity of dietary blueberry against estrogen-mediated breast cancer, J Agric Food Chem, 62 (2014) 3963-3971.

[131] J. He, M.M. Giusti, Anthocyanins: natural colorants with health-promoting properties, Annual review of food science and technology, 1 (2010) 163-187.

[132] L.-S. Wang, G.D. Stoner, Anthocyanins and their role in cancer prevention, Cancer Letters, 269 (2008) 281-290.

[133] F. Aqil, J. Jeyabalan, H. Kausar, R. Munagala, I.P. Singh, R. Gupta, Lung cancer inhibitory activity of dietary berries and berry polyphenolics, Journal of Berry Research, 6 (2016) 105-114.

[134] C.A. Schneider, W.S. Rasband, K.W. Eliceiri, NIH Image to ImageJ: 25 years of image analysis, Nat Meth, 9 (2012) 671-675.

[135] G. Zhang, B. Svenungsson, A. Karnell, A. Weintraub, Prevalence of enterotoxigenic Bacteroides fragilis in adult patients with diarrhea and healthy controls, Clinical infectious diseases : an official publication of the Infectious Diseases Society of America, 29 (1999) 590-594.

[136] K.-J. Rhee, S. Wu, X. Wu, D.L. Huso, B. Karim, A.A. Franco, S. Rabizadeh, J.E. Golub, L.E. Mathews, J. Shin, R.B. Sartor, D. Golenbock, A.R. Hamad, C.M. Gan, F. Housseau, C.L. Sears, Induction of Persistent Colitis by a Human Commensal, Enterotoxigenic Bacteroides fragilis, in Wild-Type C57BL/6 Mice, Infection and immunity, 77 (2009) 1708-1718. 
[137] S. Wu, J. Powell, N. Mathioudakis, S. Kane, E. Fernandez, C.L. Sears, Bacteroides fragilis enterotoxin induces intestinal epithelial cell secretion of interleukin-8 through mitogen-activated protein kinases and a tyrosine kinaseregulated nuclear factor-kappaB pathway, Infection and immunity, 72 (2004) $5832-5839$.

[138] T. Gu, M. De Jesus, H.C. Gallagher, T.P. Burris, N.K. Egilmez, Oral IL-10 suppresses colon carcinogenesis via elimination of pathogenicCD4+ T-cells and induction of antitumor CD8+ T-cell activity, Oncolmmunology, (2017) e1319027.

[139] G. Warnes, B. Bolker, T. Lumley, gplots: Various R programming tools for plotting data. $\mathrm{R}$ package version 2.6.0.

[140] R. Team, RStudio: Integrated Development for R., RStudio, Inc., Boston, MA, 2016.

[141] D. Sarkar, Lattice: multivariate data visualization with R, Springer Science \& Business Media2008.

[142] F. Aqil, J. Jeyabalan, A.K. Agrawal, A.H. Kyakulaga, R. Munagala, L. Parker, R.C. Gupta, Exosomal delivery of berry anthocyanidins for the management of ovarian cancer, Food \& function, 8 (2017) 4100-4107.

[143] L. Muller, P. Bednar, P. Bartak, K. Lemr, J. Sevcik, Estimation of partition coefficients by MEKC part 2: anthocyanins, Journal of separation science, 28 (2005) 1285-1290.

[144] R. Munagala, F. Aqil, J. Jeyabalan, A.K. Agrawal, A.M. Mudd, A.H. Kyakulaga, I.P. Singh, M.V. Vadhanam, R.C. Gupta, Exosomal formulation of anthocyanidins against multiple cancer types, Cancer Lett, 393 (2017) 94-102.

[145] T.C. Wallace, M.M. Giusti, Anthocyanins, Advances in Nutrition, 6 (2015) 620-622.

[146] A.E. Moran, D.H. Hunt, S.H. Javid, M. Redston, A.M. Carothers, M.M. Bertagnolli, Apc deficiency is associated with increased Egfr activity in the intestinal enterocytes and adenomas of C57BL/6J-Min/+ mice, The Journal of biological chemistry, 279 (2004) 43261-43272.

[147] L. Chung, E. Thiele Orberg, A.L. Geis, J.L. Chan, K. Fu, C.E. DeStefano Shields, C.M. Dejea, P. Fathi, J. Chen, B.B. Finard, A.J. Tam, F. McAllister, H. Fan, X. Wu, S. Ganguly, A. Lebid, P. Metz, S.W. Van Meerbeke, D.L. Huso, E.C. Wick, D.M. Pardoll, F. Wan, S. Wu, C.L. Sears, F. Housseau, Bacteroides fragilis Toxin Coordinates a Pro-carcinogenic Inflammatory Cascade via Targeting of Colonic Epithelial Cells, Cell host \& microbe, 23 (2018) 203-214.e205. 
[148] H. Sano, Y. Kawahito, R.L. Wilder, A. Hashiramoto, S. Mukai, K. Asai, S. Kimura, H. Kato, M. Kondo, T. Hla, Expression of Cyclooxygenase-1 and -2 in Human Colorectal Cancer, Cancer research, 55 (1995) 3785-3789.

[149] R.A. Gupta, R.N. DuBois, Colorectal cancer prevention and treatment by inhibition of cyclooxygenase-2, Nature Reviews Cancer, 1 (2001) 11.

[150] S.P. Claus, H. Guillou, S. Ellero-Simatos, The gut microbiota: a major player in the toxicity of environmental pollutants?, 3 (2017) 17001.

[151] L.A. Beamish, A.R. Osornio-Vargas, E. Wine, Air pollution: An environmental factor contributing to intestinal disease, Journal of Crohn's \& colitis, 5 (2011) 279-286.

[152] P.-L. Wei, S.-Y. Lin, Y.-J. Chang, Cigarette Smoking and Colorectal Cancer: From Epidemiology to Bench, Journal of Experimental \& Clinical Medicine, 3 (2011) 257-261.

[153] D.L. Diggs, A.C. Huderson, K.L. Harris, J.N. Myers, L.D. Banks, P.V. Rekhadevi, M.S. Niaz, A. Ramesh, Polycyclic aromatic hydrocarbons and digestive tract cancers: a perspective, Journal of environmental science and health. Part C, Environmental carcinogenesis \& ecotoxicology reviews, 29 (2011) 324-357.

[154] R.S. Clark, S.T. Pellom, B. Booker, A. Ramesh, T. Zhang, A. Shanker, M. Maguire, P.D. Juarez, M.J. Patricia, M.A. Langston, M.Y. Lichtveld, D.B. Hood, Validation of research trajectory 1 of an Exposome framework: Exposure to benzo(a)pyrene confers enhanced susceptibility to bacterial infection, Environmental research, 146 (2016) 173-184.

[155] J. Sun, I. Kato, Gut microbiota, inflammation and colorectal cancer, Genes \& diseases, 3 (2016) 130-143.

[156] B.C. Casto, L.A. Kresty, C.L. Kraly, D.K. Pearl, T.J. Knobloch, H.A. Schut, G.D. Stoner, S.R. Mallery, C.M. Weghorst, Chemoprevention of oral cancer by black raspberries, Anticancer research, 22 (2002) 4005-4015.

[157] H.S. Aiyer, R.C. Gupta, Berries and ellagic acid prevent estrogen-induced mammary tumorigenesis by modulating enzymes of estrogen metabolism, Cancer prevention research (Philadelphia, Pa.), 3 (2010) 727-737.

[158] G.K. Russell, R.C. Gupta, M.V. Vadhanam, Effect of phytochemical intervention on dibenzo[a,I]pyrene-induced DNA adduct formation, Mutat Res, 774 (2015) 25-32.

[159] A. Srovnalova, M. Svecarova, M.K. Zapletalova, P. Anzenbacher, P. Bachleda, E. Anzenbacherova, Z. Dvorak, Effects of anthocyanidins and 
anthocyanins on the expression and catalytic activities of CYP2A6, CYP2B6, CYP2C9, and CYP3A4 in primary human hepatocytes and human liver microsomes, J Agric Food Chem, 62 (2014) 789-797.

[160] G. Lala, M. Malik, C. Zhao, J. He, Y. Kwon, M.M. Giusti, B.A. Magnuson, Anthocyanin-rich extracts inhibit multiple biomarkers of colon cancer in rats, Nutrition and cancer, 54 (2006) 84-93.

[161] C.Y. Li, S. Lee, S. Cade, L.-J. Kuo, I.R. Schultz, D.K. Bhatt, B. Prasad, T.K. Bammler, J.Y. Cui, Novel Interactions between Gut Microbiome and Host DrugProcessing Genes Modify the Hepatic Metabolism of the Environmental Chemicals Polybrominated Diphenyl Ethers, Drug Metabolism and Disposition, 45 (2017) 1197.

[162] G. Housman, S. Byler, S. Heerboth, K. Lapinska, M. Longacre, N. Snyder, S. Sarkar, Drug Resistance in Cancer: An Overview, Cancers, 6 (2014) 17691792.

[163] B. Mansoori, A. Mohammadi, S. Davudian, S. Shirjang, B. Baradaran, The Different Mechanisms of Cancer Drug Resistance: A Brief Review, Advanced Pharmaceutical Bulletin, 7 (2017) 339-348.

[164] J.P. Hernandez, L.C. Mota, W.S. Baldwin, Activation of CAR and PXR by Dietary, Environmental and Occupational Chemicals Alters Drug Metabolism, Intermediary Metabolism, and Cell Proliferation, Current pharmacogenomics and personalized medicine, 7 (2009) 81-105.

[165] S. Wu, K.J. Rhee, E. Albesiano, S. Rabizadeh, X. Wu, H.R. Yen, D.L. Huso, F.L. Brancati, E. Wick, F. McAllister, F. Housseau, D.M. Pardoll, C.L. Sears, A human colonic commensal promotes colon tumorigenesis via activation of T helper type 17 T cell responses, Nat Med, 15 (2009) 1016-1022.

[166] S. Sakurai, T. Shimizu, U. Ohto, The crystal structure of the AhRR-ARNT heterodimer reveals the structural basis of the repression of AhR-mediated transcription, The Journal of biological chemistry, 292 (2017) 17609-17616.

[167] L. Cheng, L. Qian, G.S. Wang, X.M. Li, X.P. Li, Genetic association of aromatic hydrocarbon receptor and its repressor gene polymorphisms with risk of rheumatoid arthritis in Han Chinese populations, Medicine, 96 (2017) e6392.

[168] Y. Zhang, M. Elgizouli, B. Schottker, B. Holleczek, A. Nieters, H. Brenner, Smoking-associated DNA methylation markers predict lung cancer incidence, Clinical epigenetics, 8 (2016) 127.

[169] S.E. Bojesen, N. Timpson, C. Relton, G. Davey Smith, B.G. Nordestgaard, AHRR (cg05575921) hypomethylation marks smoking behaviour, morbidity and mortality, Thorax, 72 (2017) 646-653. 
[170] M. Mandl, M.K. Lieberum, R. Depping, A HIF-1alpha-driven feed-forward loop augments HIF signalling in Hep3B cells by upregulation of ARNT, Cell death \& disease, 7 (2016) e2284.

[171] S. Chen, M.F. Yueh, C. Bigo, O. Barbier, K. Wang, M. Karin, N. Nguyen, R.H. Tukey, Intestinal glucuronidation protects against chemotherapy-induced toxicity by irinotecan (CPT-11), Proceedings of the National Academy of Sciences of the United States of America, 110 (2013) 19143-19148.

[172] L.T. Stiemsma, L.A. Reynolds, S.E. Turvey, B.B. Finlay, The hygiene hypothesis: current perspectives and future therapies, Immunotargets and Therapy, 4 (2015) 143-157.

[173] D. Cutler, G. Miller, The Role of Public Health Improvements in Health Advances: The Twentieth-Century United States, Demography, 42 (2005) 1-22.

[174] M. Scudellari, News Feature: Cleaning up the hygiene hypothesis, Proceedings of the National Academy of Sciences, 114 (2017) 1433.

[175] J.I. Keenan, F.A. Frizelle, Bacteria flying under the radar: linking a bacterial infection to colon carcinogenesis, Infectious agents and cancer, 9 (2014) 31.

[176] L.A. Tesmer, S.K. Lundy, S. Sarkar, D.A. Fox, Th17 cells in human disease, Immunological Reviews, 223 (2008) 87-113.

[177] P.R. Burkett, G. Meyer zu Horste, V.K. Kuchroo, Pouring fuel on the fire: Th17 cells, the environment, and autoimmunity, The Journal of Clinical Investigation, 125 (2015) 2211-2219.

[178] Y. Okada, Y. Tsuzuki, H. Sato, K. Narimatsu, R. Hokari, C. Kurihara, C. Watanabe, K. Tomita, S. Komoto, A. Kawaguchi, S. Nagao, S. Miura, Trans fatty acids exacerbate dextran sodium sulphate-induced colitis by promoting the upregulation of macrophage-derived proinflammatory cytokines involved in T helper 17 cell polarization, Clinical and Experimental Immunology, 174 (2013) 459-471. [179] M. Kleinewietfeld, A. Manzel, J. Titze, H. Kvakan, N. Yosef, R.A. Linker, D.N. Muller, D.A. Hafler, Sodium Chloride Drives Autoimmune Disease by the Induction of Pathogenic Th17 Cells, Nature, 496 (2013) 518-522.

[180] C.M. Wilke, K. Bishop, D. Fox, W. Zou, Deciphering the role of Th17 cells in human disease, Trends in Immunology, 32 (2011) 603-611.

[181] S. Nakae, Y. Iwakura, H. Suto, S.J. Galli, Phenotypic differences between Th1 and Th17 cells and negative regulation of Th1 cell differentiation by IL-17, Journal of leukocyte biology, 81 (2007) 1258-1268. 
[182] A.L. Geis, H. Fan, X. Wu, S. Wu, D.L. Huso, J.L. Wolfe, C.L. Sears, D.M. Pardoll, F. Housseau, Regulatory T cell response to enterotoxigenic Bacteroides fragilis colonization triggers IL-17-dependent colon carcinogenesis, Cancer discovery, 5 (2015) 1098-1109.

[183] H. Li, J.A. Bradbury, R.T. Dackor, M.L. Edin, J.P. Graves, L.M. DeGraff, P.M. Wang, C.D. Bortner, S. Maruoka, F.B. Lih, D.N. Cook, K.B. Tomer, A.M. Jetten, D.C. Zeldin, Cyclooxygenase-2 Regulates Th17 Cell Differentiation during Allergic Lung Inflammation, American Journal of Respiratory and Critical Care Medicine, 184 (2011) 37-49.

[184] M. Bertha, E. Bellaguara, T. Kuzel, S. Hanauer, Checkpoint InhibitorInduced Colitis: A New Type of Inflammatory Bowel Disease?, ACG Case Reports Journal, 4 (2017) e112.

[185] S. Dai, R. Jia, X. Zhang, Q. Fang, L. Huang, The PD-1/PD-Ls pathway and autoimmune diseases, Cellular Immunology, 290 (2014) 72-79.

[186] Y. Zhang, C.A. Ma, M.G. Lawrence, T.J. Break, M.P. O'Connell, J.J. Lyons, D.B. López, J.S. Barber, Y. Zhao, D.L. Barber, A.F. Freeman, S.M. Holland, M.S. Lionakis, J.D. Milner, PD-L1 up-regulation restrains Th17 cell differentiation in STAT3 loss- and STAT1 gain-of-function patients, The Journal of experimental medicine, 214 (2017) 2523-2533.

[187] C.J. Hawkey, COX-2 chronology, Gut, 54 (2005) 1509.

[188] R.E. Harris, Cyclooxygenase-2 (cox-2) blockade in the chemoprevention of cancers of the colon, breast, prostate, and lung, Inflammopharmacology, 17 (2009) 55-67.

[189] V. Prima, L.N. Kaliberova, S. Kaliberov, D.T. Curiel, S. Kusmartsev, COX2/mPGES1/PGE<sub>2</sub> pathway regulates PD-L1 expression in tumor-associated macrophages and myeloid-derived suppressor cells, Proceedings of the National Academy of Sciences, 114 (2017) 1117-1122.

[190] G. Botti, F. Fratangelo, M. Cerrone, G. Liguori, M. Cantile, A.M. Anniciello, S. Scala, C. D'Alterio, C. Trimarco, A. lanaro, G. Cirino, C. Caracò, M. Colombino, G. Palmieri, S. Pepe, P.A. Ascierto, F. Sabbatino, G. Scognamiglio, COX-2 expression positively correlates with PD-L1 expression in human melanoma cells, Journal of Translational Medicine, 15 (2017) 46.

[191] C. Göbel, F. Breitenbuecher, H. Kalkavan, P.S. Hähnel, S. Kasper, S. Hoffarth, K. Merches, H. Schild, K.S. Lang, M. Schuler, Functional expression cloning identifies COX-2 as a suppressor of antigen-specific cancer immunity, Cell Death \&Amp; Disease, 5 (2014) e1568. 
[192] K. Boniface, K.S. Bak-Jensen, Y. Li, W.M. Blumenschein, M.J. McGeachy, T.K. McClanahan, B.S. McKenzie, R.A. Kastelein, D.J. Cua, R. de Waal Malefyt, Prostaglandin E2 regulates Th17 cell differentiation and function through cyclic AMP and EP2/EP4 receptor signaling, The Journal of experimental medicine, 206 (2009) 535-548.

[193] P.D. Houghteling, W.A. Walker, Why is initial bacterial colonization of the intestine important to infants' and children's health?, Journal of pediatric gastroenterology and nutrition, 60 (2015) 294-307.

[194] A.M. Berg, C.P. Kelly, F.A. Farraye, Clostridium difficile Infection in the Inflammatory Bowel Disease Patient, Inflammatory Bowel Diseases, 19 (2013) 194-204.

[195] L.G. Albenberg, G.D. Wu, Diet and the Intestinal Microbiome: Associations, Functions, and Implications for Health and Disease, Gastroenterology, 146 (2014) 1564-1572.

[196] P. Smith-Brown, M. Morrison, L. Krause, P.S.W. Davies, Dairy and plant based food intakes are associated with altered faecal microbiota in 2 to 3 year old Australian children, Scientific reports, 6 (2016) 32385.

[197] M. van Voorhis, S. Knopp, W. Julliard, J.H. Fechner, X. Zhang, J.J. Schauer, J.D. Mezrich, Exposure to Atmospheric Particulate Matter Enhances Th17 Polarization through the Aryl Hydrocarbon Receptor, PloS one, 8 (2013) e82545.

[198] A.A. Soshilov, M.S. Denison, Ligand promiscuity of aryl hydrocarbon receptor agonists and antagonists revealed by site-directed mutagenesis, Molecular and cellular biology, 34 (2014) 1707-1719.

[199] B. Lamas, J.M. Natividad, H. Sokol, Aryl hydrocarbon receptor and intestinal immunity, Mucosal Immunology, 11 (2018) 1024-1038.

[200] M. Veldhoen, K. Hirota, A.M. Westendorf, J. Buer, L. Dumoutier, J.C. Renauld, B. Stockinger, The aryl hydrocarbon receptor links TH17-cell-mediated autoimmunity to environmental toxins, Nature, 453 (2008) 106-109. 
CURRICULUM VITAE

\author{
Ashley Marie Mudd \\ Graduate Research Assistant \\ Department of Pharmacology and Toxicology \\ 580 S. Hancock St. \\ Louisville, KY 40292
}

\title{
EDUCATION:
}

University of Louisville

M.S. and Ph.D. in Pharmacology and Toxicology

Cumulative Grade Point Average: 3.8

2014-present

Brown University

A.M. in Chemistry

Cumulative Grade Point Average: 3.8

Graduation Month/Year: June 2012

Wilson College

Chemistry (with honors in the major) \& Spanish Double Major

Biology, Business and Economics Minors

Cumulative Grade Point Average: 3.9

Graduation Month/Year: June 2009

Mercersburg Academy

Graduation Month/Year: May 2005

\section{RESEARCH AND PROFESSIONAL EXPERIENCE:}

\section{Graduate Research Assistant, University of Louisville (2014-present)}

Working in the lab of Dr. Ramesh Gupta, my research focuses on utilizing Anthos and ExoAnthos in the treatment and prevention of breast and colorectal cancer as well as familial adenomatous polyposis. Additionally, my doctoral project focuses on studying the interaction between the gut bacteria (Enterotoxigenic $b$. fragilis) and the environmental pollutant, $\mathrm{B}[\mathrm{a}] \mathrm{P}$ in the development of $\mathrm{CRC}$ and how, mechanistically, the blueberry-derived non-toxic anthocyanidins (Anthos) impacts this dialogue. The results attained from this project will be a simple yet 
elegant game changer for the prevention of colorectal cancer caused by imbalance of the gut bacteria and exposure to cancer causing pollutants.

Scientist and Project lead, NuLabel Technologies (10/2012-06/2014)

Responsible for developing both the research and business aspects of my project. During this time, I also co-authored three patents.

Visiting Scholar, Department of Molecular Biology, Cell Biology and Biochemistry, Brown University (05/2013-06/2014)

Research focusing on discovering the biological phosphorylation networks in immune cells, especially $\mathrm{T}$ cell signaling pathway quantitative phosphoproteomic study.

Research Assistant, Department of Chemistry, Brown University (2010) My research focused on profiling cell wall carbohydrate changes using GC-MS during pollen development. I was responsible for developing extraction, sample preparation and GC-MS methods.

Honors Undergraduate Research, Wilson College (2008-2009)

Independent research project that focused on investigating the relationship between levels of $\beta$-carotene and Tetrachloroisophthalonitrile Fungicide Residue levels in D.carota and $R$. sativus.

Internship with the Crawford Beck Vineyard Inc. (Summer 2008)

Responsible for creating a vineyard expansion plan involving the selection of variety, clone, rootstock, site, and trellising system based upon market forces, soil chemistry, degree growing days, as well as other factors.

\section{TEACHING EXPERIENCE:}

\section{Teaching Assistant Undergraduate Organic Chemistry CHEM 350 and CHEM 360}

Brown University, Fall 2010-Spring 2012

Responsible for teaching 1-2 laboratory classes per week for the undergraduate first (CHEM 350) and second semester (CHEM 360) organic chemistry classes. During this time, I have instructed students how to use GC, prepare and run basic gravity column chromatography, perform fractional, simple and steam distillations, TLC, IR, refractometer, melting point apparatus and how to interpret the results from the above. I have also helped students interpret Mass Spectra and NMR (proton and ${ }^{13} \mathrm{C}$ ) spectra, as well as how to prepare the samples for each method. Furthermore, I have also been responsible for grading lab reports, lab quizzes, student laboratory notebooks, and lecture exams. 


\section{Peer Teacher First Year Seminar Solving 21st Century Problems: Theory to Practice, Honors Section \\ Wilson College, Fall 2007}

Worked with faculty member to develop the course, co-taught lectures, and participated in grading.

\section{Peer Teacher First Year Seminar on Sustainable Living, Honors \\ Section}

Wilson College, Fall 2006

Worked with faculty member from the Biology department to develop the course, co-taught lectures, and participated in grading.

\section{ACADEMIC AND PROFESSIONAL HONORS:}

--Currently a recipient of funding on the UofL NIEHS T32 training program in Environmental Health Sciences award

-University of Louisville $1^{\text {st }}$ place Louisville Women in Medicine and Science Research Award (Fall 2015)

-University of Louisville IPIBS Fellowship recipient (Fall 2014-Summer 2016)

-Honors in Chemistry

-Wilson College Davison Greenawalt Grove Award for Distinction in Senior Research in the Physical and Life Sciences (Spring 2009)

-Wilson College Margaret Strode Haines Award for "outstanding qualities of scholarship, interest in the humanities and strength of mind, body and spirit" (Spring 2009)

-Phi Beta Kappa honor society

-Wilson College Mary-Eleanor Maule Travel Grant (Spring 2009)

-Grant from the Pennsylvania Academy of Science for my Senior Research project entitled: An Inquiry into the Existence of a Relationship between $\beta$ carotene and Tetrachloroisophthalonitrile Fungicide Residue levels in D.carota (2008-2009)

-Scholarship to attend the 2008 International Diffuse Reflectance Conference (IDRC)

-Peer Teacher Recognition Award (Spring 2008)

-Wilson College Mary-Eleanor Maule Travel Grant for Spanish (Spring 2008) 
-National Science Foundation SEBCLAR scholarship (Fall 2007)

-Scholarship to attend Mises University at the Ludwig von Mises Institute of Austrian

Economics (Summer 2007)

-Liberty Fund weekend colloquia (Fall 2006-Spring 2009)

-Wilson College Robert Shannon McElwain Prize in Mathematics awarded (Spring 2007)

-Wilson College Organic Chemistry Award (Spring 2007)

-Wilson College Peer Teacher Recognition Award (Spring 2007)

-2006-2007 Intercollegiate Studies Institute Honors Fellow

-Wilson College Phoenix Scholar (2006-2009)

-Wilson College's Honors Program (2006-2009)

-College Board Advanced Placement Scholar with Distinction (Spring 2005)

-Mercersburg Academy Colonel Wills First Place Prize in the Study of Economics (Spring 2005)

-Maintained Honors-High Honors Academic Status while attending The Mercersburg Academy (Spring 2005)

\section{PUBLICATIONS:}

Yang, L., Li, C., Mudd, A., Gu, X. LncRNA PVT1 predicts prognosis and regulates tumor growth in prostate cancer. Biosci. Biotechnol. Biochem., 2017, 81(12) 2301-2306

Belmont, J., Gu, T., Mudd, A., Salomon, A. A PLC-y1 Feedback Pathway Regulates Lck Substrate Phosphorylation at the T Cell Receptor and SLP-76 Complex. J. Proteome Res., 2017 16(8), 2729-2742

Munagala, R., Aqil, F., Jeyabalan, J., Agrawal, A., Mudd, A. M., Kyakulaga, A. H., Vadhanam, M. V., Gupta, R. C. Exosomal formulation of anthocyanidins against multiple cancer types. Cancer Lett., 393 (2017) pp. 94-102

Manuscripts under review/currently preparing manuscripts regarding: 
Mudd, A. M., Gupta, R.C. et al., Influence of Anthos on EGFR, Src and downstream signaling and Modulation of inflammatory environment and PD-L1 expression

Mudd, A. M., Gupta, R.C. et al., The role of anthocyanidins in reequilibrating metabolic shifts induced by dysbiosis of the gut microbiome and the ubiquitous environmental carcinogen, Benzo[a]pyrene.

\section{PATENTS:}

Mudd, A.M., Munnelly, H., Lux, B., Shear Stress-Resistant Systems and Uses Thereof. Patent US20160331087 A1 (Issued Nov 17, 2016)

Cho, D., Mudd, A.M., Sonshine, D., Two-Component Hybrid Labeling System for Use in Repeat Labeling. U.S. Patent US20140072748 A1 Sept 12, 2013 (Issued March 13, 2014)

Lux, B., Mudd, A.M., Munnelly, H., Articles de conditionnement refermables et leurs procédés de fabrication et d'utilisation. WO 2015073566 A1 Nov 12, 2014. (Issued May 21, 2015)

\section{ABSTRACTS/POSTER PRESENTATIONS:}

Barbour, C. E., Mudd, A. M., Kyakulaga, A., Gupta, R. C. (2018, August) Role of anthocyanidins on immune checkpoint protein, PD-L1 in HCT116 and HT-29 colorectal cancer cells. Abstract submitted for poster session at annual UofL R25 $\mathrm{NCl}$ summer scholar poster session, Louisville, $\mathrm{KY}$.

Mudd, A. M., Gu, T., Jeyabalan, J., Munagala, R., Egilmez., N. K., Gupta, R. C. (2018, April) Novel mechanistic insight into the prevention and treatment of colorectal cancer and familial adenomatous polyposis by anthocyanidins. Abstract accepted for presentation at annual American Association for Cancer Research (AACR) meeting Chicago, IL.

Mudd, A. M., Gu, T., Jeyabalan, J., Munagala, R., Egilmez., N.K., Gupta, R. C. (2017, March)

Prevention and Treatment of Colorectal Cancer by BilberryDerived Anthocyanidin $s$ (Anthos) and Nano-Anthos. Abstract submitted for poster session at Berry Health Benefits Symposium, Pismo Beach, CA.

Mudd, A. M., Gu, T., Jeyabalan, J., Munagala, R., Egilmez., N. K., Gupta, R. C. (2016, October) Prevention and treatment of familial adenomatous polyposis 
(FAP) and colorectal cancer by bilberry-derived anthocyanidins Abstract submitted for poster session at annual Research Louisville, Louisville, KY.

Mudd, A. M., Munagala, R., Gupta, R. C. (2015, October) Exosomal formulations enhance therapeutic response of anthocyanidins against breast cancer. Abstract submitted for poster session at annual Research Louisville, Louisville, KY.

Gupta, R. C., Aqil, F., Vadhanam, M. V., Jeyabalan, J., Agrawal, A., Mudd, A. M., Kyakulaga, A., Karukonda, D., Spencer, W., Munagala, R. (2016, May) Milkderived exosomes - a platform nanocarrier to enhance anti-proliferative, antiinflammatory and anti-cancer activities of small drug molecules against multiple human cancers. Abstract accepted for presentation at Annual meeting of ISEV, Rotterdam, Netherlands.

Mudd, A. M., Santai, C. (2009, March) An Inquiry into the Existence of a Relationship between $\beta$-carotene and Tetrachloroisophthalonitrile Fungicide Residue levels in D.carota. Abstract accepted for presentation at annual meeting of the Pennsylvania Academy of Science, Harrisburg, PA

\section{Professional Memberships}

American Association for Cancer Research

American Chemical Society

Pennsylvania Academy of Science

Phi Beta Kappa Honor Society 